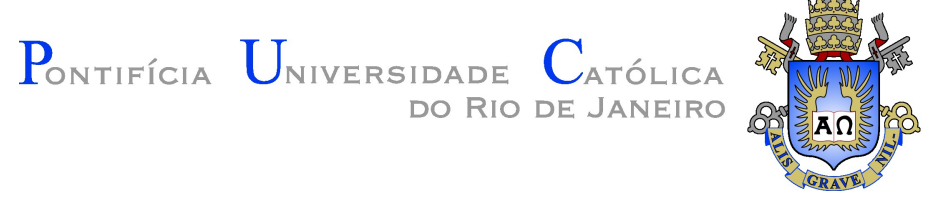

Rafael Cesar de Oliveira Góes

\title{
Linear-Elastic three-dimensional effects in notch and crack tip fields
}

\section{DISSERTAÇÃO DE MESTRADO}

Thesis presented to the Programa de Pós-graduação em Engenharia Mecânica, PUC-Rio as partial fulfillment of the requirements for the degree of Mestre em Engenharia Mecânica.

Advisor: Prof. Jaime Tupiassú Pinho de Castro 


\title{
Rafael Cesar de Oliveira Góes
}

\section{Linear-elastic three-dimensional effects in notch and crack tip fields}

Thesis presented to the Programa de Pós-Graduação em Engenharia Mecânica of the Departamento de Engenharia Mecânica do Centro Técnico Científico da PUC-Rio, as partial fulfillment of the requirements for the degree of Mestre.

\author{
Prof. Jaime Tupiassú Pinho de Castro \\ Advisor \\ Departamento de Engenharia Mecânica - PUC-Rio \\ Prof. Marco Antonio Meggiolaro \\ Departamento de Engenharia Mecânica - PUC-Rio
}

Prof. Luiz Fernando Campos Ramos Martha

Departamento de Engenharia Civil - PUC-Rio

Prof. Gustavo Henriqe Bolognesi Donato

Fundação Educacional Inaciana - FEl

Prof. José Eugenio Leal

Coordinator of the Centro Técnico Científico - PUC-Rio

Rio de Janeiro, February 18th, 2013 
All rights reserved. It is forbiden partial or complete reproduction without previous authorization of the university, the author and the advisor.

Rafael Cesar de Oliveira Góes

Rafael Góes graduated from Pontifícia Universidade Católica do Rio de Janeiro (Rio de Janeiro, Brazil) in Mechanical Engineering. He currently works in Petrobras in Subsea Pipelines Design department.

Ficha Catalográfica

Góes, Rafael Cesar de Oliveira

Linear-Elastic three-dimensional effects in notch and crack tip fields / Rafael Cesar de Oliveira Góes; orientador: Jaime Tupiassú Pinho de Castro. — 2013.

82 f: il. (color.); $30 \mathrm{~cm}$

Dissertaçao (mestrado) - Pontifícia Universidade Católica do Rio de Janeiro, Departmento de Engenharia Mecânica, 2013.

Inclui bibliografia

1. Engenharia Mecânica - Teses. 2. campos 3D em ponta de entalhe. 3. campos 3D em ponta de trinca. 4. curvatura da frente da trinca. 5. restrição material. I. Castro, Jaime Tupiassú Pinho de. II. Pontifícia Universidade Católica do Rio de Janeiro. Departmento de Engenharia Mecânica. III. Título. 


\section{Acknowledgments}

The author is much thankful for Cornell University Fracture Group, which provided a licence of FRANC3D software for use in this work, and for PETROBRAS S.A. for the financial support during the last months of research. 


\section{Abstract}

Góes, Rafael Cesar de Oliveira; Castro, Jaime Tupiassú Pinho de. Linear-Elastic three-dimensional effects in notch and crack tip fields. Rio de Janeiro, 2013. 82p. MSc Thesis - Departmento de Engenharia Mecânica, Pontifícia Universidade Católica do Rio de Janeiro.

Notches and cracks are usually treated as two-dimensional problems in most structural design and analysis tasks, employing 2D limit solutions obtained from plane elasticity theories to evaluate the severity of stress/strain concentration effects around their tips. However, due to restrictions to the Poisson strains induced by the stress gradients around such tips, these regions may be affected by important three-dimensional effects that can affect their stress/strain fields and possibly lead to non-conservative damage and life predictions if neglected. Fatigue crack initiation, plastic zone size and shape estimation, and plane stress/plane strain dominance issues on $K$-controlled fields are typical examples of problems sensible to such effects. Linear Elastic Finite Element techniques are used to simulate 3D effects along notch fronts, such as how the thicknessto-notch root radius $B / \rho$ affects the stress and strain fields that surround them. The influence of such 3D effects is evaluated from the structural design point of view. Then versatile submodeling techniques are used to study similar 3D effects along the fronts of short and long cracks. Finally, a stepwise remeshing routine is used to show how an initially straight crack must slightly curve its front during its propagation by fatigue.

\section{Keywords}

3D notch tip fields; 3D crack tip fields; crack front curvature; material constraint. 


\section{Resumo}

Góes, Rafael Cesar de Oliveira; Castro, Jaime Tupiassú Pinho de. Efeitos tridimensionais lineares elásticos em campos em torno de pontas de entalhes e trincas. Rio de Janeiro, 2013. 82p. Dissertação de Mestrado - Departamento de Engenharia Mecânica, Pontifícia Universidade Católica do Rio de Janeiro.

Entalhes e trincas são normalmente tratados como problemas bi-dimensionais na maioria das análises e projetos, com o emprego de soluções limites 2D obtidas de teorias de elasticidade plana para avaliar a severidade dos efeitos de concentração de tensão e deformação próximo à sua ponta. Contudo, devido à restrição por deformações de Poisson induzidas pelos gradientes de tensão em torno da ponta, estas regiões podem sofrer efeitos tridimensionais importantes em seus campos de tensão e deformação, os quais, se negligenciados, podem levar a predições não conservasdoras de dano e vida. A iniciação de trincas por fadiga, estimativas de tamanho e formato de zona plástica e dominância de estado plano de tensão ou deformação em campos controlados por $K$ são exemplos típicos de problemas sensíveis a tais efeitos. Técnicas de Elementos Finitos Linear Elástico são utilizadas na simulação de efeitos 3D ao longo da frente de entalhes, tais como a influência da razão espessura-raio-dearredondamento sobre os campos de tensão e deformação que a cercam. A influência de tais efeitos 3D é examinada do ponto de vista de projeto estrutural. Então, a versátil técnica da submdelagem é empregada no estudo de efeitos 3D similares ao longo da frente de trincas curtas e longas. Finalmente, uma rotina de remalhamento passo-a-passo é utilizada para demonstrar como uma trinca inicialmente reta deve se curvar conforme propaga por fadiga.

\section{Palavras-chave}

campos 3D em ponta de entalhe; campos 3D em ponta de trinca; curvatura da frente da trinca; restrição material. 


\section{Contents}

\begin{tabular}{lr}
\hline List of Figures & 9
\end{tabular}

\begin{tabular}{lr}
\hline List of Tables & 12
\end{tabular}

\begin{tabular}{lll}
\hline 1 & Introduction & 15
\end{tabular}

$\begin{array}{lll}1.1 & \text { Three-dimensional effects on notch-tip fields } & 15\end{array}$

1.2 Three-dimensional effects in crack tip-fields 22

$\begin{array}{lll}1.3 & \text { Objectives } & 26\end{array}$

$\begin{array}{lll}1.4 & \text { Main contributions } & 27\end{array}$

$\begin{array}{lll}1.5 & \text { Summary } & 27\end{array}$

$\begin{array}{lll}2 & \text { Methodology } & 30\end{array}$

$\begin{array}{lll}2.1 & \text { Notch FE models } & 31\end{array}$

2.2 3D crack FE models 35

$\begin{array}{lll}2.2 .1 & \text { Submodeling technique } & 35\end{array}$

2.2 .2 2D global model 36

2.2 .3 3D submodels 38

$\begin{array}{lll}2.3 & \text { Crack growth model } & 42\end{array}$

\begin{tabular}{lll}
\hline & Results & 46
\end{tabular}

$\begin{array}{lll}3.1 & 3 \mathrm{D} \text { effects on notch tip fields } & 46\end{array}$

$\begin{array}{lll}3.1 .1 & 3 \mathrm{D} \text { effects on notch design procedures } & 53\end{array}$

$\begin{array}{lll}3.2 & \text { Crack analyses results } & 55\end{array}$

3.3 Crack front curvature development 66

\begin{tabular}{lll}
\hline 4 & Conclusions & 72
\end{tabular}

\begin{tabular}{lll}
\hline 5 & Bibliography & 74
\end{tabular}

\begin{tabular}{lll}
\hline A Notch FE models data & 78
\end{tabular}

\begin{tabular}{lll}
\hline B & Crack submodels runs & 82
\end{tabular} 


\section{List of Figures}

$1.12 \mathrm{D}$ representation of $\sigma_{0}$ classical notch tip stress 16

1.2 Pioneer analytical solutions for 2D stress concentration problems: Circular (Kirsch) and Elliptical (Inglis) hole at infinite plate. The coordinate system origin is placed at the center of the circular or elliptical hole. 16

1.3 3D representation of notched plate under uni-axial load: the coordinate system's origin is placed at the center of the notch tip. The presence of the notch causes a stress gradient ahead of the tip and, due to the Poisson's contraction, the stress state of an element close to the notch tip will be 3D. 19

$1.4 \quad$ Notch configurations analysed by Guo et al. [16] 20

1.5 Cracked plate with thickness $B$ and crack length $a$ loaded in pure mode-I by nominal stress $\sigma_{n}$, with coordinate system origin at the center of the crack tip.

$1.6 \quad$ Nakamura and Parks FE model for $K_{I}$ dominated region around crack tip, see [19, 22]

2.120 -node brick element

2.2 2D Stress distribution ahead of EH tip with apect ratio $t=b / a=$ \begin{tabular}{ll|l|l|}
$0.5, \rho / a=0.25:$ Inglis $|11|$ analytical solution and 2D FE results & 34
\end{tabular}

2.3 3D FE model of notched plate with $b / a=0.5$ and $B / \rho=4$. For SE cases, $1 / 4$ symmetry (with respect to planse $x y$ and $x z$ ); for EH cases, $1 / 8$ symmetry $(x y, x z$ and $y z))$.

2.4 (a) Number of nodes used in SE and EH notch analyses and (b) CPU time demanded for analysis

$2.5 \quad K_{\sigma_{\max }} / K_{\sigma_{\text {surf }}}$ and $K_{\sigma_{\text {surf }}} / K_{t}$ VS $B / \rho$ for EH: comparison of present results and those obtained by She and Guo, given by eq. 2.1 to 2.2

2.6 Submodeling technique applied in the evaluation of 3D effects close to crack tips in globally pl- $\sigma$ loaded specimen

2.7 (a) Global Model Mesh using pl- $\sigma$ elements and (b) detail of the mesh close to the crack tip

2.8 Quarter Point Elements around crack tip

2.9 Collapse of a 20-node brick element into a prismatic element (source: |12])

2.10 Quarter-point collapsed brick elements around the crack front 39

2.11 Mesh of a submodel with $B / a=0.6 \quad 40$

2.12 Mesh of a submodel with $B / a=0.6$ close to the crack tip 40

2.13 Submodel $B / a=10$, convergence check: $K_{I} / K_{I_{2 D}}$ VS $z / B$ distribution along the crack front for several refinements along $z$-direction.

2.14 Submodel $B / a=10$, convergence check: $K_{I_{\mathrm{mp}}}, K_{I_{\max }}$ and $K_{I_{\mathrm{surf}}}$ variation with the number of elements used in $z$-direction 
2.15 Submodel $B / a=10$, convergence check: $\sigma_{y_{\mathrm{mp}}}$ gradient ahead of crack tip 42

$\begin{array}{lll}2.16 & \text { Crack propagation model } & 45\end{array}$

$3.1 \quad K_{\varepsilon} / K_{t}(\mathrm{a})$ and $K_{\sigma} / K_{t}(\mathrm{~b})$ along $z / B$ for an Elliptic Hole with

\begin{tabular}{|ll|}
\hline$b / a=0.5, \rho / a=0.25$ & 47
\end{tabular}

$3.2 \quad K_{\sigma_{\max }} / K_{t}$ and $K_{\varepsilon_{\max }} / K_{t}$ variation with $B / \rho$ for elliptical holes 48

$3.3 z / B$ Position of $K_{\varepsilon_{\max }} / K_{t}$ and $K_{\sigma_{\max }} / K_{t}$ for different $B / a$ values 48

\begin{tabular}{|ll}
3.4 & Variation of $K_{\sigma_{\max }} / K_{t}, K_{\sigma_{\mathrm{mp}}} / K_{t}$, and $K_{\sigma_{\text {surf }}} / K_{t}$ with the thick- \\
\hline & ness to root radius ratio $B / \rho$ for the elliptical holes
\end{tabular}

$3.5 T_{z 0} / T_{z 0_{\mathrm{mp}}}$ vs. $z / B$ for the semi-elliptical notch with $\rho / a=0.01 \quad 50$

\begin{tabular}{lll}
\hline 3.6 & $T_{z 0_{\mathrm{mp}}} / \nu$ versus $B / \rho$ for elliptical and semi-elliptical notches $\quad 50$ \\
\hline
\end{tabular}

$3.7 \quad$ Normalized gradient $\sigma_{y_{\mathrm{mp}}} / \sigma_{y 0_{\mathrm{mp}}}$ vs $x / \rho$ for several notches with different $\rho / a$ ratios 51

$3.8 \sigma_{y} / \sigma_{y 0}$ vs. $x / \rho$ for an elliptical hole with $\rho / a=0.25$ and

\begin{tabular}{|c|c|c|}
\hline$B / a=12$ at different $z / B$ positions & 52 \\
\hline
\end{tabular}

3.9 Normalized out-of-plane constraint factor $T_{z_{\mathrm{mp}}} / T_{z 0_{\mathrm{mp}}}$ ahead of

\begin{tabular}{|lll}
\hline & the notch tips & 52 \\
\hline 3.10 & $\sigma_{\text {Mises }} / K_{t} \sigma_{n}$ as a function of $T_{z 0}$, for various $\sigma_{y 0}$ values & 54
\end{tabular}

$3.11 K_{\sigma_{\max }}, K_{\sigma_{\mathrm{mp}}}$, and $K_{\sigma_{\mathrm{Mises}}}=\sigma_{\mathrm{Mises}} / K_{t} \sigma_{n}$ as a function of $B / \rho \quad 55$

$3.12 \quad K_{I} / K_{I_{2 D}}$ distribution along the crack front for several $B / a$ ratios.

\begin{tabular}{|l|l|}
\hline \multicolumn{3}{|c|}{ The solutions are compared with She \& Guo's fit expression (eq. } \\
\hline 1.18$)$ for $K_{I} / K_{I_{2 D}}$ along the front of a long crack. \\
\hline
\end{tabular}

\begin{tabular}{lll}
3.13 & $z / B$ coordinate along the crack front where the maximum SIF & \\
\hline$K_{I_{\max }}$ occurs & 57
\end{tabular}

\begin{tabular}{|ll}
3.14 & $T$-stress $/ K_{I_{2 D} \sqrt{\pi a}}$ \\
cracks & distribution along the front of short and long \\
\hline 3.15 & $K_{I}$
\end{tabular}

$3.15 K_{I_{\max }} / K_{I_{2 D}}$ and $K_{I_{\mathrm{mp}}} / K_{I_{2 D}}$ variation with the crack size in large

\begin{tabular}{rr} 
plates & 59 \\
\hline
\end{tabular}

\begin{tabular}{|ll}
3.16 & $\sigma_{y_{\mathrm{mp}}} / \sigma_{n}$ distribution ahead of the crack tip in the large edge \\
cracked plate & 59
\end{tabular}

$3.17 \sigma_{y}(z) / \sigma_{n} \mathrm{VS} x / a$ gradient ahead of the crack tip at different $z / B$

\begin{tabular}{|l|l|}
\hline planes, in cracked plates with different $B / a$ ratios & 61
\end{tabular}

3.18 Behavior of the transversal constraint along the mid-plane of the cracked plate $T_{z_{\mathrm{mp}}} / \nu$ as a function of the relative position ahead $\begin{array}{ll}\text { of the crack tip versus } x / B & 62\end{array}$

3.19 Behavior of the transversal constraint along the mid-plane of the cracked plate $T_{z_{\mathrm{mp}}} / \nu$ as a function of the relative position ahead $\begin{array}{ll}\text { of the crack tip: } x / a & 62\end{array}$

3.20 Example of toughness $K_{c}$ dependence on the specimen thickness $B$ for a Ti-6Al-6V-2Sn alloy (see $[26 \mid)] 63$

3.21 Iso-lines where $T_{z}=0.5$ along the thickness of the plate for several cracked plates with different $B / a$ ratios $\quad 64$

3.22 Area ahead of the crack where $T_{z}>\gamma \cdot \nu$ normalized by $a^{2}$ versus $B / a$

3.23 Area ahead of the crack where $T_{z}>\gamma \cdot \nu$ normalized by $B^{2}$ versus $B / a \quad 65$ $\begin{array}{lll}3.24 & \text { Area ahead of the crack where } T_{z}>\gamma \cdot \nu \text { normalized by } B \cdot a & \\ \text { versus } B / a\end{array}$ 
3.25 Crack front shape evolution for $a_{0} / B=0.02$ and $n=2 \quad 67$

3.26 Crack front shape evolution for $a_{0} / B=0.02$ and $n=4$

3.27 Slightly curved front shapes under steady growth conditions 68

$3.28\left(a_{\max }-a_{\min }\right) / B$ variation as the crack grows from an initially straight front $a_{0}$. 69

$3.29 K_{I_{\max }} / K_{I_{2 D}}$ VS. $\left(a_{\text {surf }}-a_{0}\right) / B \quad 70$

$\begin{array}{lll}3.30 & \text { Incidence angle } \beta \text { at free surface with crack propagation } & 71\end{array}$ 


\section{List of Tables}

$\begin{array}{lll}2.1 & \text { Computer characteristics } & 31\end{array}$

2.2 Correction term $\phi$ for SE notch SCF 33

2.3 Notch FE model parameters 33

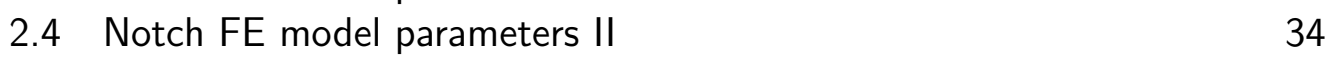

2.5 Crack growth model parameters 44

3.1 Transient distance $\Delta a_{\text {trans }} / B$ covered by the crack before achieve$\begin{array}{ll}\text { ment of steady front shape for different initial lengths } a_{0} / B & 69\end{array}$

\begin{tabular}{lll}
\hline A.1 & EH model runs: $\rho / a=1.0$ & 78
\end{tabular}

$\begin{array}{lll}\text { A.2 } & \text { EH model runs: } \rho / a=0.25 & 79\end{array}$

$\begin{array}{lll}\text { A.3 } & \text { EH model runs: } \rho / a=0.04 & 79\end{array}$

\begin{tabular}{lll}
\hline A.4 & EH model runs: $\rho / a=0.01$ & 79
\end{tabular}

A.5 SE model runs: $\rho / a=1.0 \quad 80$

A.6 SE model runs: $\rho / a=0.25$

A.7 SE model runs: $\rho / a=0.04 \quad 80$

A.8 SE model runs: $\rho / a=0.01$

B.1 Crack submodels 82 


\section{Nomenclature}

\section{List of symbols}

Symbol
$\sigma$
$\varepsilon$
$a$
$a_{0}$
$b$
$B$
$E$
$G$
$H$
$K$
$K_{I}, K_{I I}, K_{I I I}$
$K_{t}$
$K_{\sigma}$
$K_{\varepsilon}$
$n$
$r$
$S_{Y}$
$t$
$x, y, z$
$T_{z}$
$\beta$

W

$\varepsilon$

$\Phi_{Y}$

\section{Description}

stress

strain

Crack length or notch depth; ellipsis major semiaxis

Crack initial length

Ellipsis minor semi-axis

Plate thickness

Modulus of elasticity

Shear modulus

Plate half-height

Stress intensity factor

Stress intensity factor for modes $I, I I$ and $I I I$ respectively

$2 \mathrm{D}$ stress/strain concentration factor

3D stress concentration factor

3D strain concentration factor

Paris' propagation rule exponent

Radial cylindrical coordinate

Material's yield strength

Ellipsis aspect ratio, $t=b / a$

Cartesian coordinates

Out-of-plane constraint factor

Angle with which the crack front intersects the specimen's free surface

In EH models, plate half-width; in SE and SE(T) specimen models, plate width

Strain

Safety factor against yielding 


$\begin{array}{ll}\nu & \text { Poisson's ratio } \\ \rho & \text { Notch tip roundness radius } \\ \sigma & \text { Stress } \\ \sigma_{n} & \text { Nominal stress } \\ \sigma_{\text {Mises }} & \text { Von Mises' equivalent stress } \\ \sigma_{\text {Tresca }} & \text { Tresca's equivalent stress } \\ \theta & \text { Angular cyclindrical coordinate } \\ (*)_{0} & \text { Property }(*) \text { occurring at the notch tip; except } \\ (*)_{\mathrm{mp}} & \text { when }(*)=a, \text { in this case, see } a_{0} \\ & \text { Property }(*) \text { occurring at the middle-plane (or } \\ (*)_{\operatorname{surf}} & \text { symmetry plane) of the specimen } \\ & \text { Property }(*) \text { occurring at the free surface of the } \\ (*)_{\max } & \text { specimen } \\ (*)_{\min } & \text { Maximum value of property }(*) \\ & \text { Minimum value of property }(*)\end{array}$

\section{List of acronyms}

2D

$3 \mathrm{D}$

$\mathrm{EH}$

FE

FM

LE

LEFM

$\mathrm{pl}-\varepsilon$

pl- $\sigma$

$p z$

$\mathrm{SCF}$

SE

$\mathrm{SE}(\mathrm{T})$

$\mathrm{SIF}$ two-dimensional

three-dimensional

Elliptical Hole

Finite Element

Fracture Mechanics

Linear Elastic

Linear Elastic Fracture Mechanics

Plain-strain

Plain-stress

Plastic zone

Stress Concentration Factor

Semi-Elliptical (notch)

Single-Edge Tensile

Stress Intensity Factor 


\section{1}

\section{Introduction}

The calculation of stress and strain fields around notches is a frequent matter in engineering. Notches usually behave as localized stress raisers, very likely to originate cracks or cause creeping, yielding, environment assisted cracking and other failure mechanisms driven by stresses.

Catalogs for Stress Concentration Factors (SCF) and Stress Intensity Factors (SIF), see ahead, list solutions obtained from analytical, numerical, and/or experimental methods, usually treating the stress analysis problem as if it was two-dimensional (2D), assuming Linear Elastic (LE) plane stress (pl- $\sigma$ ) or plane strain $(\mathrm{pl}-\varepsilon)$ conditions around the notch tip.

Nevertheless, recent literature shows that the material close to notches and crack tips is subjected to material constraint effects, and the stress state is in fact three-dimensional (3D). The present work investigates, through 3D Finite Element (FE) analyses, LE 3D effects acting on the stress and strain fields of notched and cracked uni-axially tensiled plates with finite thickness and, in case of cracks, the influence of such effects on the development of the crack front shape while it propagates.

Along this chapter, a brief bibliographic revision presents a background on the 3D notch problem (section 1.1) and 3D crack problem (section 1.2). Section 1.3 summarizes the objectives of this work and, in section 1.4 , its main contributions are listed.

\section{1 .}

\section{Three-dimensional effects on notch-tip fields}

For design purposes, the maximum stress $\sigma_{0}$ that acts at notch tip is usually calculated by using a Stress Concentration Factor (SCF) $K_{t}$ to multiply the nominal stress $\sigma_{n}$ that would act there if the notch had no effect on the stress and strain fields that surround them (see Figure 1.1):

$$
\sigma_{0}=K_{t} \sigma_{n}
$$




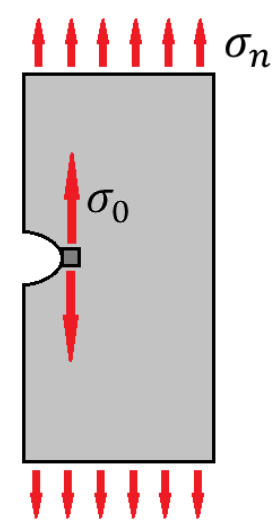

Figure 1.1 - 2D representation of $\sigma_{0}$ classical notch tip stress

SCF's are usually dependent on geometry and load acting on the specimen. The first analytical solution for LE stress/strain fields around notches was obtained by Kirsch in 1898 [14], for an infinite plate with a circular hole, described by:

$$
\begin{array}{rlrl}
\sigma_{r}= & & \frac{\sigma_{n}}{2}\left(1-\frac{\rho^{2}}{r^{2}}\right)+\left(1-\frac{4 \rho^{2}}{r^{2}}+\frac{3 \rho^{4}}{r^{4}}\right) & \cos 2 \theta \\
\sigma_{\theta}= & \frac{\sigma_{n}}{2}\left(1-\frac{\rho^{2}}{r^{2}}\right)-\left(1+\frac{3 \rho^{4}}{r^{4}}\right) \cos 2 \theta \\
\tau_{r \theta}= & -\frac{\sigma_{n}}{2}\left(1-3 \frac{\rho^{4}}{r^{4}}+2 \frac{\rho^{2}}{r^{2}}\right) \sin 2 \theta
\end{array}
$$

with cartesian and cylindrical coordinate systems origins placed at the center of the circular hole, see Figure $1.2(\mathrm{a})$.
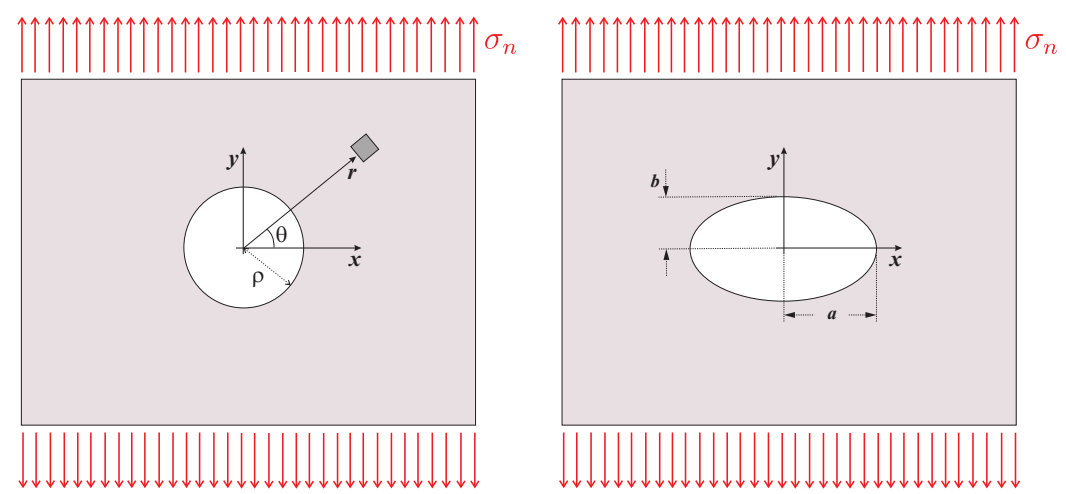

1.2(a): Kirsch plate: circular hole 1.2(b): Inglis plate: elliptical hole at infinite plate at infinite plate

Figure 1.2 - Pioneer analytical solutions for 2D stress concentration problems: Circular (Kirsch) and Elliptical (Inglis) hole at infinite plate. The coordinate system origin is placed at the center of the circular or elliptical hole.

In the notch root, where $r=\rho$, the ratio $\sigma_{\theta} / \sigma_{n}$ becomes 3 , the well known value of $K_{t}$ for the circular hole in infinite plate. Regarding the $x$ direction 
ahead of the notch root, eq. 1.2 can be rewriten in the alternative form ( $\mathrm{Li}$ et al, [16]):

$$
\begin{aligned}
& \sigma_{x}=\frac{K_{t} \sigma_{n}}{2}\left[\left(1+\frac{x}{\rho}\right)^{-2}-\left(1+\frac{x}{\rho}\right)^{-4}\right] \\
& \sigma_{y}=\frac{K_{t} \sigma_{n}}{3}\left[1+\frac{1}{2}\left(1+\frac{x}{\rho}\right)^{-2}+\frac{3}{2}\left(1+\frac{x}{\rho}\right)^{-4}\right]
\end{aligned}
$$

Later, Inglis in 1913 [11] presented the exact analytical solution for the elastic field around an elliptical hole in an infinite plate under multi-axial load, for which the Kirsch solution is a particular case. The general solution obtained for the multi-axial problem is not trivial, and the multi-axial load case is out of the scope of the present work. Instead, let us consider the plate loaded in mode I, where the nominal stress $\sigma_{n}$ is applied perpendicular to the major semi-axis $(a)$ of the ellipsis. Considering that the ellipsis semi-axes $a$ and $b$ are parallel to cartesian axe $x$ and $y$ respectively (see Figure $1.2(\mathrm{~b})$, the $\sigma_{y}$ stress component ahead of the hole root can be described by:

$$
\sigma_{y}=\sigma_{n}\left[1+\frac{\left(a^{2}-2 a b\right)\left(x-\sqrt{x^{2}-a^{2}+b^{2}}\right)+a b^{2}(a-b) x}{(a-b)^{2}\left(x^{2}-a^{2}+b^{2}\right) \sqrt{x^{2}-a^{2}+b^{2}}}\right]
$$

Also, in the ellipsis vertice $(x, y)=(a, 0)$, equation 1.5 becomes:

$$
\sigma_{y}=\sigma_{n}(1+2 \sqrt{a / \rho})
$$

For analysis purposes, equation 1.6 becomes a fine approximation for $K_{t}$ of any notch that fits within depth $a$ and tip-radius $\rho$ :

$$
K_{\text {tapprox }}=(1+2 \sqrt{a / \rho})
$$

Both presented examples are exact within the infinite medium assumption and $2 D$ limitation. Other analytical solutions were obtained for stress concentrations, but most of them equally limited to infinite geometries and are based in $2 D$, plane or axi-symmetric geometries. 
Creager and Paris [5] presented a method to estimate the $K_{t}$ of a notch from the SIF of a similar crack as $K_{t} \approx 2 K_{I} / \sigma_{n} \sqrt{\pi \rho}$, but, again, most of the avaiable SIF catalogs are based on 2D solutions, see [25].

Later Glinka and Newport [8] used this solution to estimate the stress fields ahead of relatively deep notches, obtaining:

$$
\begin{gathered}
\sigma_{x}=\frac{K_{t} \sigma_{n}}{2 \sqrt{2}}\left[\left(\frac{1}{2}+\frac{x}{\rho}\right)^{-1 / 2}-\frac{1}{2}\left(\frac{1}{2}+\frac{x}{\rho}\right)^{-3 / 2}\right] \\
\sigma_{y}=\frac{K_{t} \sigma_{n}}{2 \sqrt{2}}\left[\left(\frac{1}{2}+\frac{x}{\rho}\right)^{-1 / 2}+\frac{1}{2}\left(\frac{1}{2}+\frac{x}{\rho}\right)^{-3 / 2}\right]
\end{gathered}
$$

It is worth mentioning that Kirsch Plate and the deep notch can be taken as two conceptual limit solutions for plain notches concerning the ratio $a / \rho$ : in the first, the notch depth is equal to the notch tip radius $(a / \rho=1)$, while in the second, the notch depth is much larger then the tip radius $(a / \rho \rightarrow \infty)$.

With the advent of Finite Elements and the boost of numerical computational capacity, SCF's were obtained for a wide variety of geometries under various loading conditions although, once again, mostly restricted to $2 D$ domain. Many of them are related in Peterson's Stress Concentration Factors [20], a widely used catalog of SCF's.

But the 2D representation of a notched component presents limitations, even in the simplest cases. Consider, for instance, the case of a notched tensioned plate with uniform thickness, where the material far from the notch is subjected to dominating plane-stress (pl- $\sigma$ ) conditions, with $\sigma_{y}=\sigma_{n}$ and the remaining stress components $\sigma_{x}=\sigma_{z}=\tau_{x y}=\tau_{y z}=\tau_{x z}=0$. The strains at the notch tip are $\varepsilon_{y}=\sigma_{n} / E$ and $\varepsilon_{x}=\varepsilon_{z}=-\nu \sigma_{n} / E$, which are easily obtained from Hooke's Law for the LE case:

$$
\begin{array}{r}
\varepsilon_{x}=\left[\sigma_{x}-\nu\left(\sigma_{y}+\sigma_{z}\right)\right] / E \\
\varepsilon_{y}=\left[\sigma_{y}-\nu\left(\sigma_{x}+\sigma_{z}\right)\right] / E \\
\varepsilon_{z}=\left[\sigma_{z}-\nu\left(\sigma_{x}+\sigma_{y}\right)\right] / E \\
\varepsilon_{x y}=\frac{\sigma_{x y}}{2 G}, \varepsilon_{y z}=\frac{\sigma_{y z}}{2 G}, \varepsilon_{x z}=\frac{\sigma_{x z}}{2 G}
\end{array}
$$

Using a coordinate system centered at the notch root, see Fig. 1.3 (from now on, this reference system shall be used for every notch analysis), the classical LE 2D approach expressed by eq. 1.1 results in $\sigma_{y}(x=y=0)=\sigma_{0}=$ 


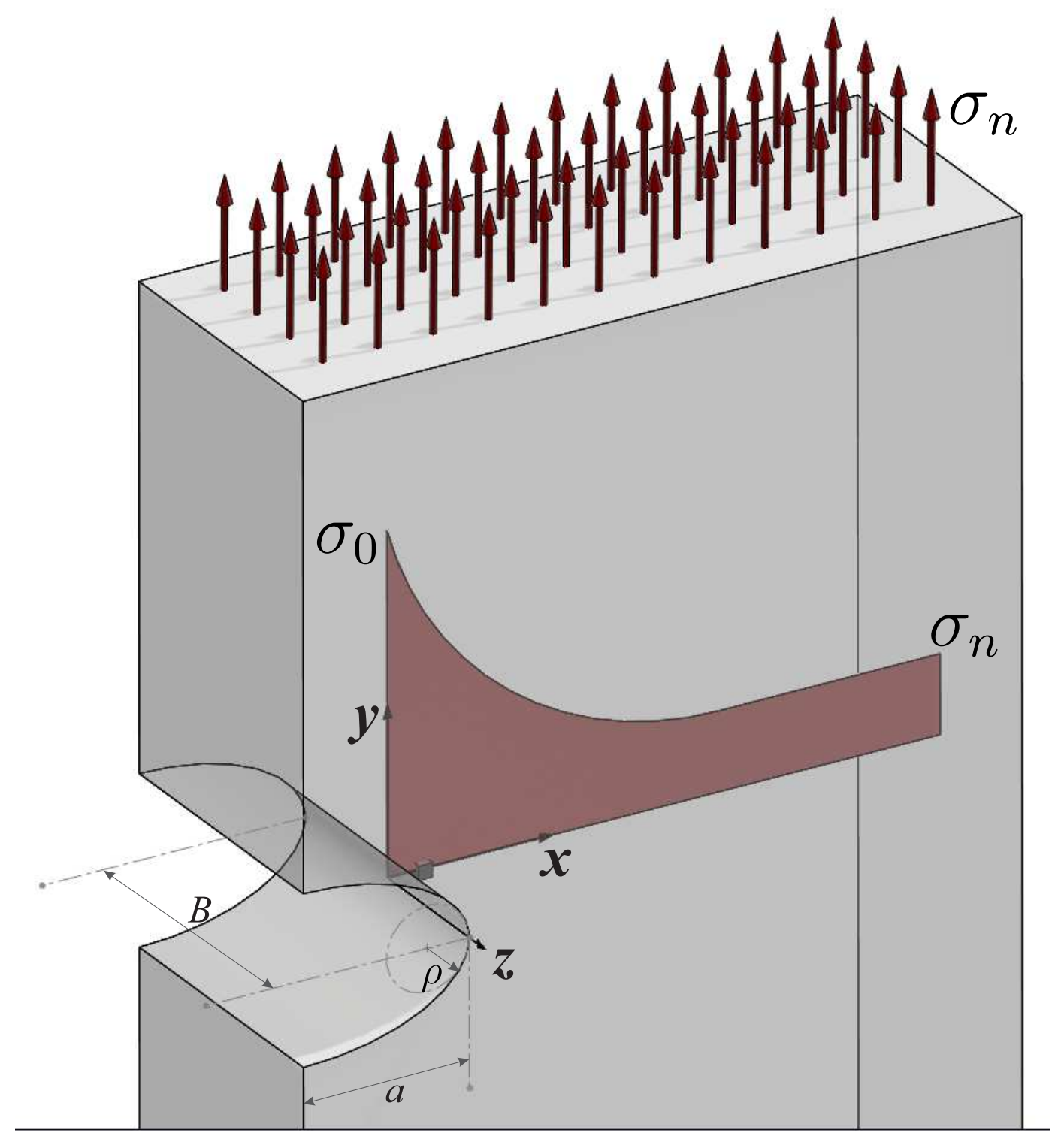

Figure 1.3 - 3D representation of notched plate under uni-axial load: the coordinate system's origin is placed at the center of the notch tip. The presence of the notch causes a stress gradient ahead of the tip and, due to the Poisson's contraction, the stress state of an element close to the notch tip will be 3D. 
$K_{t} \sigma_{n}, \varepsilon_{y}=K_{t} \sigma_{n} / E$, and $\varepsilon_{x}=\varepsilon_{z}=-\nu K_{t} \sigma_{n} / E$. But the tendency of the notch root to have a much higher transversal strain $\varepsilon_{z}$ than the rest of the piece is restrained by it. This restriction generates a tensile $\sigma_{z}$ component around the notch root. Hence, the stress and strain fields close to notch tips must in fact be $3 \mathrm{D}$, due to the restriction to the Poisson contraction induced by the stress/strain gradients that act there. To quantify this restriction, a transversal constraint factor $T_{z}$ can be defined at any given point by the ratio between the out-of-plane stress $\sigma_{z}$ and the sum of the in-plane stress components $\sigma_{x}$ and $\sigma_{y}$ that act there. Under pl- $\sigma$ limit condition, the only non-null stress components are $\sigma_{x}$ and $\sigma_{y}$, which means that $T_{z}=0$ in such cases, whereas under plane-strain (pl- $\varepsilon$ ) limit condition, Hooke's Law leads to $T_{z}=\nu$. Thus, in resume:

$$
T_{z}=\frac{\sigma_{z}}{\sigma_{x}+\sigma_{y}}= \begin{cases}0 & \mathrm{pl}-\sigma \\ \nu & \mathrm{pl}-\varepsilon\end{cases}
$$

Youngdahl and Sternberg solved the infinite solid with a 3D ellipsoidal cavity problem, one of the very few analytical solutions available for LE 3D notch problems. They also obtained approximate solutions for the 3D stresses in an infinite plate of finite thickness with a circular hole [31].

Through the last decades, the great boost of computational capability possibilitated the solution of larger and larger numerical models. Great effort has been dedicated, since then, in obtaining solutions for the 3D fields in stress concentrators.
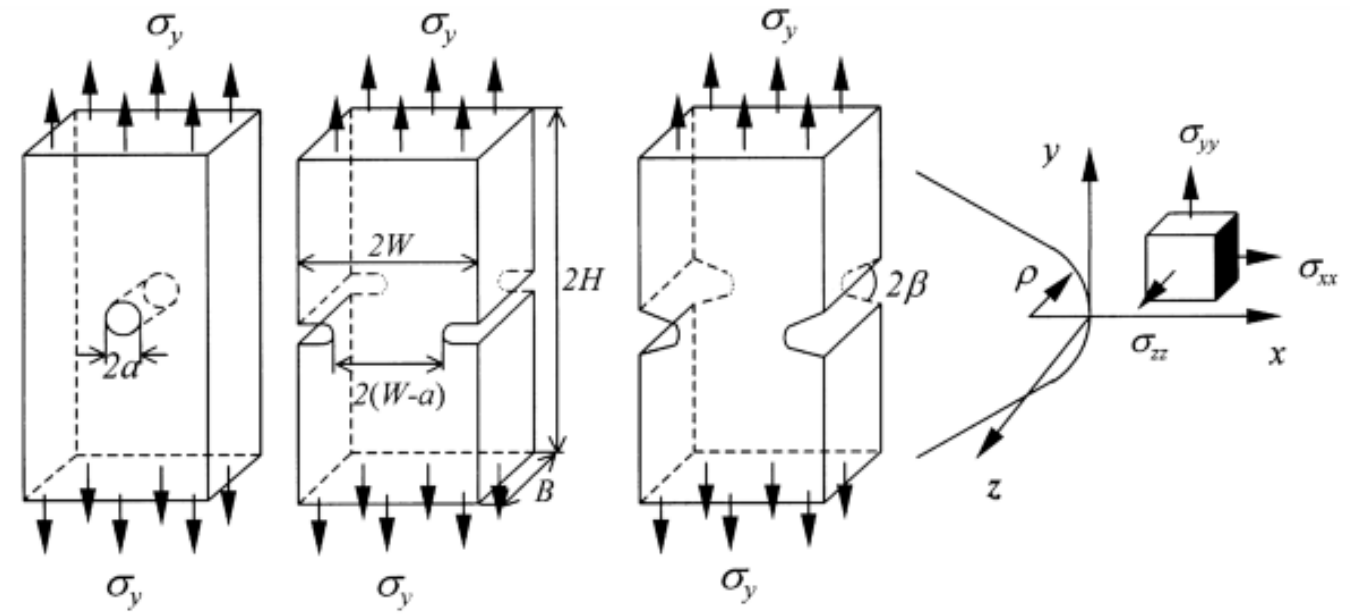

Figure 1.4 - Notch configurations analysed by Guo et al. 16

Guo et al. 16 used 3D finite elements to model the linear elastic tipfields of several notch configurations. Three basic configurations of notch in a 
plate were deeply investigated, namely: circular hole, U-notch and V-notch (see figure 1.4), varying the following parameters: the tip radius $\rho$, the normalized notch depth $a / W$ and the plate width $B$, resulting in important conclusions:

- The SCF in a 3D-notch root is closely related with the dimensionless thickness $B / \rho$ and the notch configuration. If, with respect to the tip radius $\rho$, the plate is very thin $(B / \rho \rightarrow 0)$ or very thick $(B / \rho \rightarrow \infty)$, the $\mathrm{SCF}$ will be the same as in the $2 \mathrm{D}$ pl $-\sigma$ solution. For a plate with finite thickness, the SCF's in the notch root are greater than the corresponding $2 \mathrm{D}$ value: it grows with $B / \rho$ from the plane $K_{t}$ to a peak value, after which it decreases until reaching a steady value, which depends on the $\rho / a$ ratio.

- Taking $\sigma_{y 0}(z)$ as the $\sigma_{y}$ stress component at the notch root at a particular $z$ along the thickness, the normalized stress $\sigma_{y} / \sigma_{y 0}$ along $x / \rho$ independs of $B$ and of the notch configuration, and is well predicted by the $2 \mathrm{D}$ solution.

- Strong 3D effects may exist close to a stress concentrator, and the 3D affected zone is characterized by $T_{z}>0$ (see eq. 1.11). For relatively closed notches, the size of the 3D-effect zone is insensity to the notch geometry and is about $3 B / 8$ on the mid-plane.

- No matter how thick the plate is, due to the finite radius $\rho$, unlike cracks the notch root never assumes the pl- $\varepsilon$ state.

- If $T_{z 0}$ is the out-of-plane constraint at the notch root, $\left(T_{z} / T_{z 0}\right)_{\mathrm{mp}}$ variation along normalized $x / B$ is insensitive to the notch configuration and the plate thickness, and can be well described by the equation:

$$
\left(\frac{T_{z}}{T_{z_{0}}}\right)_{\mathrm{mp}}=1-4.35\left(1+0.628 \frac{x}{B}\right)^{-2}+4.35\left(1+0.628 \frac{x}{B}\right)^{-4}
$$

Yang et al. 30 presented similar results and showed that stress and strain concentration effects are decoupled along the thickness, even within the linear elastic (LE) regime. Instead of the single SCF $K_{t}=\sigma_{\max } / \sigma_{n}$ used in 2D analyses, independent $K_{\sigma}=\sigma_{\max } / \sigma_{n}$ and $K_{\varepsilon}=\varepsilon_{\max } / \varepsilon_{n}$ stress and strain concentration factors should be considered when analyzing $3 \mathrm{D}$ notch problems.

In further works, investigating finite-thickness plates with elliptical holes, She and Guo 23] and $\mathrm{Yu}$ et al. 32 obtained the relationship between the 
maximal SCF along the notch-root thickness, the SCF at the free surface $K_{\sigma_{\text {surf }}}$ and the $2 \mathrm{D} K_{t}$. As observed, $K_{\sigma_{\text {surf }}} / K_{t}$ is a monotonic decreasing function of the dimensionless plate thickness, the ellipsis aspect-ratio $t=b / a$ and Poisson's ratio. $K_{\sigma_{\max }} / K_{\sigma_{\text {surf }}}$, on the contrary, is a monotonic increasing function of the same parameters:

$$
\begin{aligned}
K_{\sigma_{\max }} / K_{\sigma_{\text {surf }}} & =f_{1}(B / \rho, t, \nu) \\
K_{\sigma_{\text {surf }}} / K_{t} & =f_{2}(B / \rho, t, \nu)
\end{aligned}
$$

\section{2.}

\section{Three-dimensional effects in crack tip-fields}

Consider a cracked plate with thickness $B$, uni-axially loaded in modeI (y-direction) by the nominal stress $\sigma_{n}$, with the cartesian and cylindrical coordinate systems origin placed at the center of the crack tip, as illustrated in Figure 1.5 .

The 2D classical description of the LE stresses and strains ahead of a crack tip was obtained independently by Williams ( 27]) and Irwin [13. Williams used an infinite series that provides an asymptotic solution for the crack tip stress/strain fields, usually approximated by its first term, the stress intensity factor (SIF). This one-parameter SIF-based description for the stress field around crack tips loaded in mode I gives the well-known $\sigma_{i j}=\left(K_{I} / \sqrt{2 \pi r}\right) g_{i j}(\theta)$ solution, where $i, j=x, y$ :

$$
\begin{aligned}
\sigma_{x} & =\frac{K_{I}}{\sqrt{2 \pi r}} \cos \frac{\theta}{2}\left(1-\sin \frac{\theta}{2} \sin \frac{3 \theta}{2}\right) \\
\sigma_{y} & =\frac{K_{I}}{\sqrt{2 \pi r}} \cos \frac{\theta}{2}\left(1+\sin \frac{\theta}{2} \sin \frac{3 \theta}{2}\right) \\
\tau_{x y} & =\frac{K_{I}}{\sqrt{2 \pi r}} \cos \frac{\theta}{2}\left(\sin \frac{\theta}{2} \sin \frac{3 \theta}{2}\right)
\end{aligned}
$$

This classical solution is singular at the crack tip, but as infinite stresses are physically impossible, its idealized LE fields are always perturbated by some yielding (or by other non-linear deformation mechanisms) around real crack tips. In spite of this limitation, classical Linear Elastic Fracture Mechanics (LEFM) concepts are very much useful. Nevertheless, they are applicable only when the unavoidable plastic zones $(p z)$ that always accompany crack tips 
are contained within a small region close to them, i.e. when such $p z$ sizes are much smaller than the cracked component dimensions. Therefore, when LEFM concepts are employed, good estimates for $p z(\theta)$ sizes and shapes are needed to verify the consistency of their predictions.

Souza [24] recently discussed the limitations of $p z(\theta)$ estimates based on SIF alone. In particular they showed that the $T$-stress correction (the second term in the Williams series solution for the stress fields around crack tips) may not be sufficient to achieve good $p z$ size estimates in many practical cases, since the nominal stresses used in most engineering designs nowadays are typically associated to yielding safety factors $3>\phi_{Y}>1.25 \Rightarrow 0.33<\sigma_{n} / S_{Y}<0.8$, where $S_{Y}$ is the material yielding strength. Indeed, neither SIF alone nor (SIF $+\mathrm{T}$ ) approximations for the stress fields around crack tips can reproduce the nominal stress $\sigma_{n}$ far from it, and to neglect the influence of such relatively high $\sigma_{n}$ on $p z$ estimates leads to non-negligible errors. This topic is not further pursued here, since the main focus of this work is to evaluate $3 \mathrm{D}$ effects around notch and crack tips under predominantly LE conditions, such as those usually encountered in fatigue applications. Nevertheless, such $p z$ are intimately linked to the amount of material subjected to compatibility-induced constraints around the crack tip. This fact alone can raise questions such as: how do such restrictions ahead of a crack tip behave? Are such 3D effects always negligible or there are practical cases where they must be considered? If $3 \mathrm{D}$ notches present differences between the stresses in the free surface and the interior, will a crack present a similar behavior?

Concerning the crack behavior at free surfaces, Bazant and Estenssoro [2] demonstrated that the singularity along the entire crack front remains proportional to $1 / \sqrt{r}$ during fatigue crack propagation under LEFM conditions. They used a spherical hemisphere to model the region where the crack front edge meets the free surface of the cracked piece under several intersection angles $\beta$. Through eigenvalue analyses, they conclude that for a crack loaded in mode I only a single $\beta$, dependent on Poisson's ratio $\nu$, attends that singularity condition. In the particular case of a crack perpendicular to the free surface $(\beta=\pi / 2), K_{I}$ must be zero in this point, so fatigue cracks cannot propagate with a straight front.

Nakamura and Parks [19] presented valuable results after numerically analyzing 3D LE stress and strain fields around crack tips within a SIFdominated zone in a plate. The region close to the crack tip was modeled as a disk centered at the crack tip (see figure 1.6). The crack size $a$ was considered long with respect to the cracked plate thickness $(a>>B)$. The boundary of the disk $(r=R)$ was loaded by the displacement field generated by the SIF $K_{I}$ 
and $K_{I I}$ applied on the plate, using the so called Boundary Layer approach, expressed by:

$$
\begin{aligned}
& u_{i}=\sqrt{\frac{R}{2 \pi}} \frac{1+\nu}{E}\left[K_{I} f_{I_{i}}(\theta)+K_{I I} f_{I I_{i}}(\theta)\right] ; \quad i, j=1,2 \\
& \text { with }\left\{\begin{array}{l}
f_{I_{1}}(\theta)=\cos (\theta / 2)\left[\kappa-1+2 \sin ^{2}(\theta / 2)\right] \\
f_{I_{2}}(\theta)=\sin (\theta / 2)\left[\kappa+1-2 \sin ^{2}(\theta / 2)\right] \\
f_{I I_{1}}(\theta)=\sin (\theta / 2)\left[\kappa+1+2 \sin ^{2}(\theta / 2)\right] \\
f_{I I_{2}}(\theta)=\cos (\theta / 2)\left[\kappa-1+2 \sin ^{2}(\theta / 2)\right]
\end{array}\right.
\end{aligned}
$$

where $\kappa=(3-\nu) /(1+\nu)$ for pl- $\sigma$ and $\kappa=3-4 \nu$ for pl- $\varepsilon$ limit conditions.

Parks and Nakamura's model was revisited by She and Guo [22], who used a more refined FE mesh to further explore 3D effects around crack tips. Fitting expressions for their FE solutions, they proposed a 2-parameter description of the tip fields, using the SIF $K$ and the transversal constraint $T_{z}$ as parameters to describe them. Their in-plane stress components are obtained from equations very similar to the classical $K$-field description:

$$
\begin{aligned}
& \sigma_{i j}= \frac{K_{I} f_{i j}^{I}(\theta)+K_{I I} f_{i j}^{I I}(\theta)}{\sqrt{2 \pi r}}, i, j=1,2 \\
& \text { with }\left\{\begin{array}{l}
f_{11}^{I}(\theta)=\cos (\theta / 2)[1-\sin (\theta / 2) \sin (3 \theta / 2)] \\
f_{22}^{I}(\theta)=\cos (\theta / 2)[1+\sin (\theta / 2) \sin (3 \theta / 2)] \\
f_{12}^{I}(\theta)=\cos (\theta / 2) \sin (\theta / 2) \cos (3 \theta / 2) \\
f_{11}^{I I}(\theta)=-\sin (\theta / 2)[2+\cos (\theta / 2) \cos (3 \theta / 2)] \\
f_{22}^{I I}(\theta)=\sin (\theta / 2)[\sin (\theta / 2) \cos (\theta / 2) \cos (3 \theta / 2)] \\
f_{12}^{I I}(\theta)=\cos (\theta / 2)[1-\sin (\theta / 2) \sin (3 \theta / 2)]
\end{array}\right.
\end{aligned}
$$

When the cracked plate is modeled as a 3D stress analysis problem, the SIF $K_{I}$ and $K_{I I}$ around the crack tip vary through the plate thickness due to the transversal constraint effect induced by the Poisson contraction that is very much active there. These local $K_{I}$ and $K_{I I}$ are obtained from the applied 2D SIF $K_{I}^{\mathrm{far}}$ and $K_{I I}^{\mathrm{far}}$ that govern the LE fields further from the crack tip, which are exempt of such constraint effects:

$$
\begin{aligned}
K_{I}(z / B) & =K_{I}^{\mathrm{far}} F_{I}(z / B) \\
K_{I I}(z / B) & =K_{I I}^{\mathrm{far}} F_{I I}(z / B)
\end{aligned}
$$


Assuming that $T_{z_{I}}$ and $T_{z_{I I}}$ are the out-of-plane constraint factors under pure mode I and II, respectively, the transversal stress component close to the crack tip can be written as:

$$
\sigma_{z}=\frac{T_{z_{I}} K_{I}\left[f_{I_{11}}(\theta)+f_{I_{22}}(\theta)\right]+T_{z_{I I}} K_{I I}\left[f_{I I_{11}}(\theta)+f_{I I_{22}}(\theta)\right]}{\sqrt{2 \pi r}}
$$

The correction terms $F_{I}, F_{I I}, T_{z I}$ and $T_{z I I}$ are functions of $r_{0}=r / B$ and $z_{0}=2 z / B$. As the present work scope is restricted to cracks loaded in mode I, only $T_{z I}$ and $F_{I}$ are described below:

$$
\begin{gathered}
T_{z I}=\widetilde{T}_{z I}\left(r_{0}, \theta, z_{0}\right) \\
=\nu\left(1+b r_{0}^{0.5}\right) \exp \left(c r_{0}^{d}\right),-90^{\circ} \leq \theta \leq 0^{\circ} \\
\text { with }\left\{\begin{array}{r}
b=-1.518-0.342 z_{0}^{2}\left(1-z_{0}\right)^{-0.5} \\
c=-3.444-2.223 z_{0}^{3}\left(1-z_{0}\right)^{-1} \exp \left(0.062 z_{0}^{3}\right) \\
d=Q_{1} \cos \theta+Q_{2} \sin ^{2} \theta+Q_{3} \\
Q_{1}=0.82078-0.58758 z_{0}-0.13642 z_{0}^{2} \\
Q_{2}=0.0242+0.16947 z_{0}+0.0114 z_{0}^{2}-0.16721 z_{0}^{3} \\
Q_{3}=0.98172-0.23712 z_{0}-0.8194 z_{0}^{2}+0.93912 z_{0}^{3}
\end{array}\right. \\
F_{I}\left(z_{0}\right)=m_{1}\left[1-z_{0}^{m_{2}}\right]^{m 3} \\
\text { with }\left\{\begin{array}{l}
m_{1}=0.25 \nu^{1.5} \exp (1.69 \nu)+1 \\
m_{2}=2.61 \nu^{3} \exp (1.71 \nu)+3.14 \\
m_{3}=0.096 \nu \exp \left(3.95 \nu^{2}\right)
\end{array}\right.
\end{gathered}
$$

Strong 3D effects are observed within a distance $r=B / 2$ from the crack tip, and the 3D-2D transition occurs within $B / 2<r<3 B / 2$. She and Guo showed that a long crack with a straight front under pure mode-I loads presents a non-uniform $K_{I}$ distribution. At the free surfaces, $K_{I}$ must vanish, as observed by Bazant and Estenssoro [2], but in the middle plane $K_{I}$ is approximately $6.7 \%$ higher than the classical 2D solution for $\nu=0.3$.

Although ingenious, Nakamura and Parks' model presents some conceptual limitations that should not be disregarded. It assumes that the crack is much longer than the plate thickness, meaning that the model may not represent well a crack in its early stages of propagation, when its size $a$ is of the 
order of the plate thickness $B$ or even lower. Also, the response is obtained for a SIF boundary load, meaning that all limitations associated to $K$-field assumptions are incorporated by the model. For instance, all 3D correction terms are taken as functions of $r / B$ and $2 z / B$, but the $K$-dominance of the stress field is strictly valid only very close to the crack tip. These assumptions fail to describe the situation where the $3 \mathrm{D}$ affected zone surpasses the $K$-dominated region.

Furthermore, ideally straight cracks are just a convenient mathematical trick. Even if a crack could somehow be created with a straight front e.g. on plate edge, experimental observations show that its front curves when it starts to propagate, a phenomenon known as crack tunneling. Extensive research on the tunneling phenomenon [10,15,21] show that, in a through-cracked plate, the curved front shape can present a difference between its maximal and surface dimensions ( $a_{\max }$ and $a_{\text {surf }}$ ) up to $5 \%$ of the plate thickness B, according to [21]. This apparently slight curvature is shown to bring considerable impact on SIF calculations close to the free-surface.

Through a recursive remeshing algorithm, $\mathrm{Wu}[29]$ was able to numerically obtain the shape of the crack front for a given $K_{I}$ distribution in a through-cracked plate. Crack tunneling depth around $0.025 B$ was observed e.g. for the uniform $K_{I}$ distribution. He also discusses the influence of the crack length a and of the plate thickness $B$ on the crack front shape and on the $K_{I}$ differences with respect to $2 \mathrm{D}$ solutions.

\section{3. \\ Objectives}

The objectives of the present work are: the identification of the 3D effects exerted by the finite thickness in the stress/strain tip fields ahead of a single notch or crack in a plate, the achievement of a linear-elastic estimate of the limits of $K_{I}$ dominance on the stress fields ahead of a crack; the identification of similarities and divergences between the behaviour of long cracks/thin plates and shallow cracks/thick plates through the examination of the influence of the thickness-to-crack length ratio $B / a$ on the stress fields; to observe the crack front cuvature development from an initially straight crack in LE propagation under Paris' rule. 


\section{4 .}

\section{Main contributions}

This work revisits the most recent literature concepts on 3D LE notches through several FE models results, and discusses the importance of 3D effects on notch design issues. Furthermore, it obtains SIF distributions for cracked $\mathrm{SE}(\mathrm{T})$-like semi-infinite plates with several $B / a$ ratios, to evaluate their true influence on the $K_{I}$ distribution, using powerful submodeling techniques that intrinsically avoid $K$-field domination and long crack hypothesis limitations. On the sequence, the FRANC3D code 1001[4] is used to simulate the 3D growth of an initially straight front crack of initial length $a_{0}$ under simple Paris rule, to observe how the crack front shape and the $K_{I}$ distribution develop. The influence of $a_{0} / B$ and of Paris' exponent $n$ is also discussed.

\section{5 .}

\section{Summary}

This work is divided in four chapters (Introduction, Methodology, Results and Conclusions), a Bibliography Index and Appendices

Chapter 2 presents the methodology used in the examination of the 3D effects on notches and crack tip fields. Section 2.1 describes the construction of 3D FE notch models in Abaqus [6] used to reproduce She and Guo's results in [23]. Section 2.2 describes the modelling process used in the finite thickness cracked plates models in Abaqus [6], which employed the submodeling technique. Finally, section 2.3 briefly presents the models used in FRANC3D associated with Abaqus [6] to simulate the propagation of an initially straight crack in a finite-thickness plate under tension.

The results are presented and discussed in chapter 3. Section 3.1 presents the comparison of the results obtained in the present work with the recent literature on 3D notches, and the relevance of the 3D effects is discussed from a design point of view. In section 3.2 , the influece of the $B / a$ ratio on the stress fields ahead of a straight passing crack are presented and discussed. Finally, section 3.3 presents the behaviour of a passing crack, initially straight, propagating in LE regime and Paris' rule starting with different initial lengths.

The main conclusions are summarized in chapter 4.

Additionally, the appendices bring relevant data concerning the Finite Element models runs. 


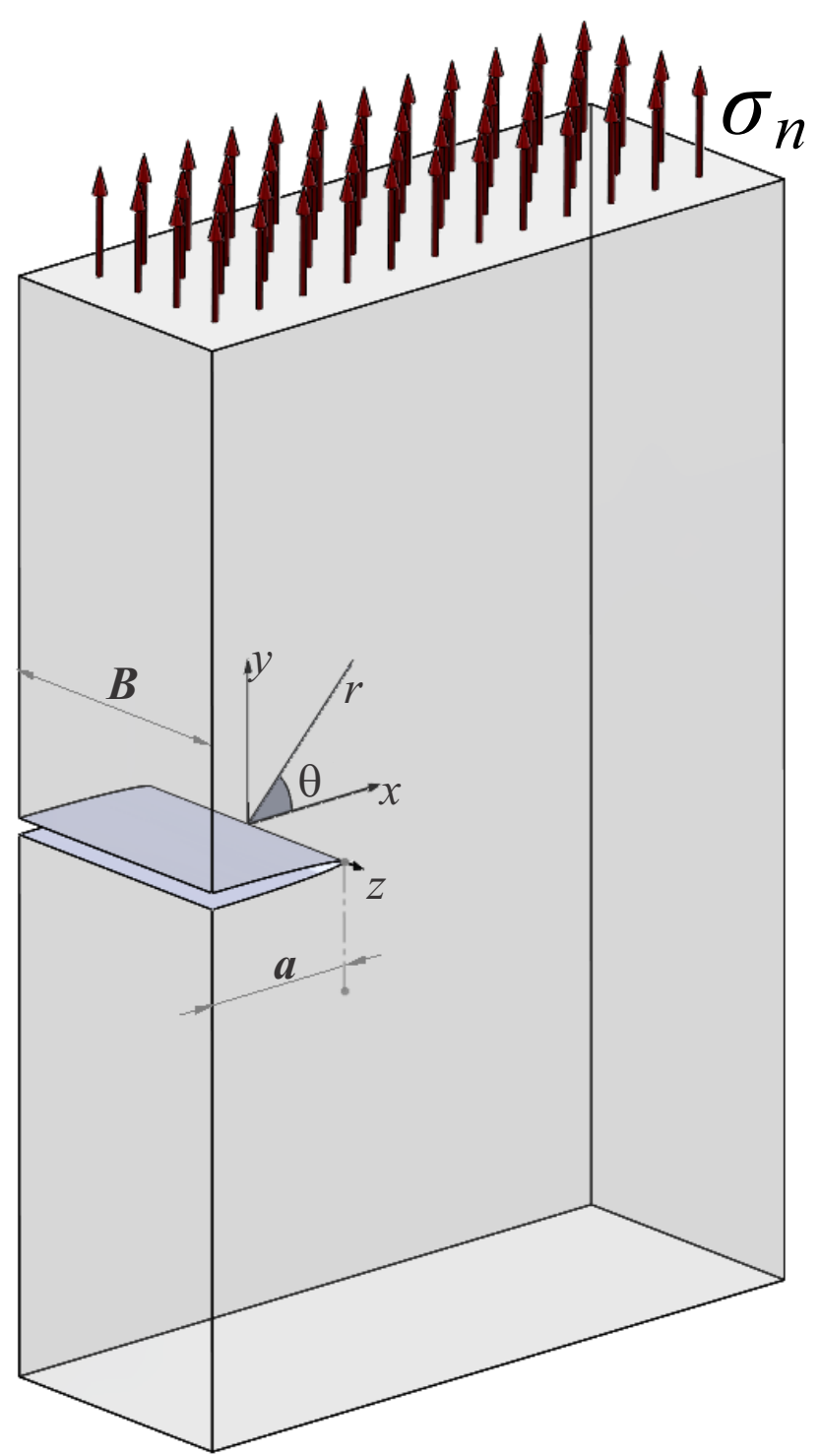

Figure 1.5 - Cracked plate with thickness $B$ and crack length $a$ loaded in pure mode-I by nominal stress $\sigma_{n}$, with coordinate system origin at the center of the crack tip. 


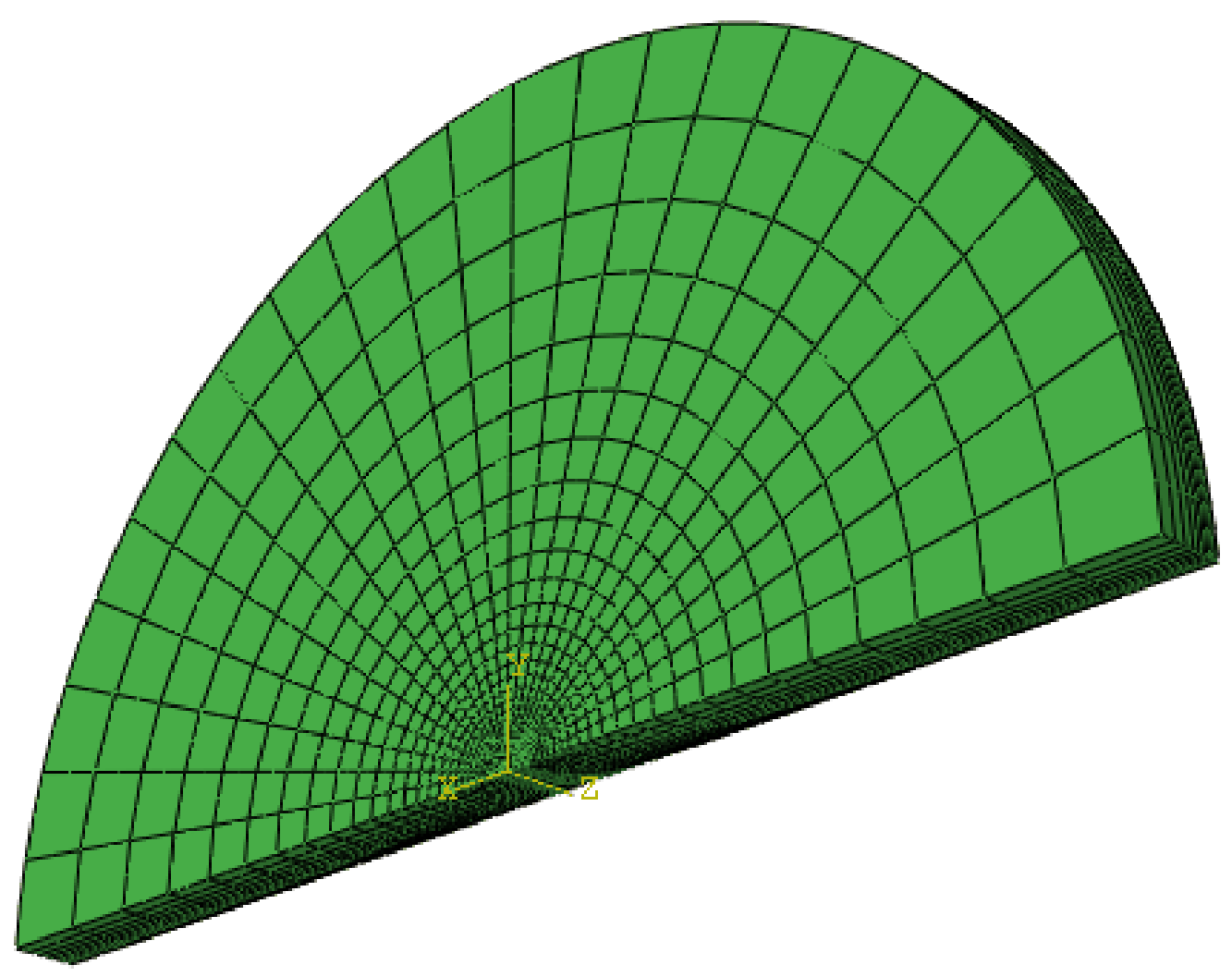

Figure 1.6 - Nakamura and Parks FE model for $K_{I}$ dominated region around crack tip, see $[19,22$ 


\section{2}

\section{Methodology}

This chapter presents the methodology used for the study of 3D effects on notch and crack tip fields, which consisted of three stages:

1. 3D FE analysis of LE tensile plates with through notches

2. 3D FE analysis of LE tensile plates with through edge cracks with straight front

3. Simulation of the propagation of an initially straight front crack under pulsating load and simple Paris' rule

The analysis of 3D fields in notches is performed for gain of confidence in the 3D FE modeling of the stress concentration problem without the presence of the singularity (crack). For that purpose, several models of plates with Elliptical Holes (EH) and Semi-Elliptical (SE) notches were analysed. Two benchmark solutions are used to check the adequacy of the models to detect 3D effects in the stress concentration: EH (Inglis' plate) and SE notch analytical solutions and She and Guo's results in [23].

After the reproduction of literature results for notches, the problem of the cracked plate is approached, introducing the singularity in the plate models. Several $B / a$ ratios are examined. In this stage, the crack is modeled with a straight front, and the Submodeling Technique (see section 2.2.1) is employed for application of the load avoiding excessive computational cost. SIF and stress fields are analysed and compared with the classical 2D $K$-solution and with the long crack solution presented in 22].

Finally, a crack growth simulation is performed for initially straight cracks, in order to observe the impacts the $3 \mathrm{D}$ effects bring to the crack front shape and to the SIF distribution along the crack front while it propagates.

All FE analyses were performed with Abaqus v6-10 software [6], using traditional quadratic continuous elements and LE regime. The analyses were run in an ASUS Laptop, with an Intel® Core $^{\mathrm{TM}} \mathrm{i}$ processor (complete specification in Table 2.1). 
Table 2.1 - Computer characteristics

\begin{tabular}{|rl|}
\hline Manufacturer: & ASUSTeK Computer Inc. \\
Model: & ASUS Notebook N53Jq \\
& Series \\
Processor: & Intel@ Core $^{\mathrm{TM}_{\mathrm{i}} \text { CPU Q740 }}$ \\
& @1.73GHz \\
Installed Memory (RAM): & $4.00 \mathrm{~GB}$ \\
System Type: & 64-bit Operating System \\
\hline
\end{tabular}

Several analyses used Abaqus [6] parallel processing tool, due to the large number of degrees of freedom used in the models.

\section{1.}

\section{Notch FE models}

Several FE models were built in order to represent infinite plates with Elliptical Holes (EH) and semi-infinite plates with Semi-Elliptical (SE) notches with finite thickness $B$. The models were built using C3D20 full integration solid element from Abaqus [6] library. C3D20 is a traditional 20-node quadratic brick, containing 3 degrees of freedom per node $\left(u_{x}, u_{y}, u_{z}\right)$.

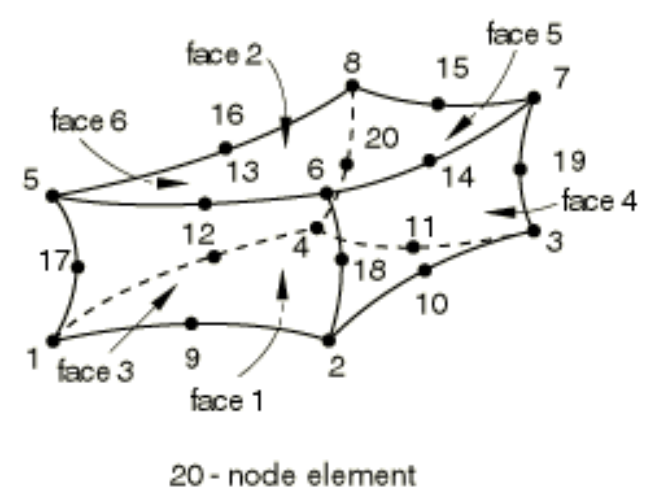

Figure 2.1 - 20-node brick element

She and Guo's results presented in 23 served as benchmark for the present models development. In their work, similar to what is described here, 3D plates of several thicknesses with elliptical holes under uni-axial tension are analysed. The relations between $K_{\sigma_{\max }}$ and $K_{\sigma_{\text {surf }}}$ with respect to the 2D $K_{t}$ are adjusted by monotonic functions of $B / \rho$ and $\rho / a$, with $t=b / a$ (the ellipsis aspect ratio), for the particular Poisson's ratio used $(\nu=0.33)$.

$$
\left.K_{\sigma_{\max }} / K_{\sigma_{\text {surf }}}(B / \rho)=a_{1}-\left(a_{1}-1\right)\left[1+(B / \rho)^{n_{1}}\right] \exp \left[b_{1}(B / \rho)^{c_{1}}\right)\right]
$$


with:

$$
\begin{gathered}
{\left[\begin{array}{l}
a_{1} \\
b_{1} \\
c_{1} \\
n_{1}
\end{array}\right]=\left[\begin{array}{l}
1.3403 t^{-0.112}-0.3009 t^{0.888}+0.3531 t^{1.888}-0.1482 t^{2.888} \\
-0.2953 t^{4}+0.3689 t^{3}-0.1832 t^{2}-0.3066 t-0.7493 \\
-0.6718 t^{4}+2.2765 t^{3}-2.9337 t^{2}+2.1754 t+0.1980 \\
0.8996-0.8366 /[1+\exp (10.8225 t-1.8301)]
\end{array}\right]} \\
\left.K_{\sigma_{\text {surf }}} / K_{t}=a_{2}-\left(a_{2}-1\right)\left[1+(B / \rho)^{n_{2}}\right] \exp \left[b_{2}(B / \rho)^{c_{2}}\right)\right]
\end{gathered}
$$

with:

$$
\left[\begin{array}{l}
a_{2} \\
b_{2} \\
c_{2} \\
n_{2}
\end{array}\right]=\left[\begin{array}{l}
\left(1.6132 t^{-1.882}+0.3757\right) \log \left(0.5137 t^{2}+1\right) \\
0.7288 t^{4}-1.7403 t^{3}+1.2620 t^{2}-0.5594 t-0.7590 \\
-2.3682 t^{4}+6.0150 t^{3}-5.5302 t^{2}+2.5281 t+0.1867 \\
t /(0.37+2.1245 t-0.8911 \sqrt{t})
\end{array}\right]
$$

The $2 \mathrm{D}$ reference $\mathrm{SCF}$ for the $\mathrm{EH}$ case $\left(K_{t_{\mathrm{EH}}}\right)$ is given by eq. 1.6 , reproduced below. The SCF for SE notch, though, has no closed form expression, but Bowie in 1966 [3] was able to fit a correction term $\phi$ to adjust the SCF of an $\mathrm{EH}\left(K_{t_{\mathrm{SE}}}\right)$ with the same $t$.

$$
\begin{aligned}
K_{t_{\mathrm{EH}}}(t) & =1+2 / t \\
K_{t_{\mathrm{SE}}}(t) & =\phi \cdot K_{t_{\mathrm{EH}}}(t)
\end{aligned}
$$

The parameters used in the models construction are presented in tables 2.3 and 2.4. The material parameters $E=200 G P a$ and $\nu=0.33$ were the same used by She and Guo in [23].

Plate width $W$ values were chosen to avoid boundary effects and to acchieve the infinite (for EH cases) and semi-infinite (for SE cases) plate condition. Every model was built with symmetry with respect to the $x y$ plane at the plate mid-thickness and to the $x z$ plane. The EH models received additional symmetry with respect to $y z$ plane. The uniform tensile load was applied as negative unitary pressure on the superior plate face $(y=H)$.

Figure 2.2 shows an example of the $\sigma_{y}$ distribution along $x$-axis obtained with a $2 \mathrm{D} \mathrm{pl-} \sigma \mathrm{EH}$ model with $\rho / a=0.5$, where the semi-infinite plane solution 
Table 2.2 - Correction term $\phi$ for SE notch SCF

\begin{tabular}{|c|c|}
\hline$t$ & $\phi$ \\
\hline 0 & 1.1215 \\
0.0526 & 1.106 \\
0.1111 & 1.092 \\
0.1765 & 1.08 \\
0.25 & 1.069 \\
0.3333 & 1.059 \\
0.4286 & 1.05 \\
0.5385 & 1.042 \\
0.6667 & 1.034 \\
0.8182 & 1.028 \\
1 & 1.022 \\
\hline
\end{tabular}

Table 2.3 - Notch FE model parameters I

\begin{tabular}{|l|c|c|}
\hline Parameter & Symbol & Value \\
\hline Poisson's ratio & $\nu$ & 0.33 \\
Young's modulus & $E$ & $200 \mathrm{GPa}$ \\
Width & $W$ & $60 \mathrm{~mm}$ \\
Height & $H$ & $60 \mathrm{~mm}$ \\
\hline
\end{tabular}

is well-reproduced, showing that the used dimensions are enough to describe the infinite plate behaviour.

The meshing process followed some common guidelines. The notch tip region was mapped-meshed, with a maximum element size of $0.1 \rho$ at the notch tip. At a certain distance from the notch tip, the number of elements through thickness was reduced in order to save computational effort. Figure 2.3 shows a particular mesh created for a Semi-Elliptical Notch model with $b / a=0.5$, $\rho / a=0.25$.

The resulting linear equation systems were solved using Abaqus [6] sparse solver. Fig. 2.4 presents the number of nodes and the CPU time used in the analysis of each model.

Fig. 2.5 clearly shows that the results obtained by the present EH models (circles) reproduce with very good agreement She and Guo's results (lines), represented by eq. 2.1 and 2.2. This successful comparison shows that the present models are able to capture the 3D effects previously observed in literature. 
Table 2.4 - Notch FE model parameters II

\begin{tabular}{|c|c|c|c|l|}
\hline Notch & $a(\mathrm{~mm})$ & $b(\mathrm{~mm})$ & $\rho / a$ & $B / \rho$ \\
\hline EL & 1 & 1 & 1 & $0.1,0.2,0.4,0.6,1,1.5,2,3,4,6,8,10,20$ \\
& 1 & 0.5 & 0.25 & $0.4,0.8,2,2.8,4,6,8,12,16,32,48$ \\
& 1 & 0.2 & 0.04 & $3,6,10,15,20,30,50,75,100$ \\
& 1 & 0.1 & 0.01 & $0.4,0.6,1,2,4,6,10,20,40,60,100$ \\
\hline SE & 1 & 1 & 1 & $0.2,0.3,0.5,0.7,1,2,3,5,7,10,20,30$ \\
& 1 & 0.5 & 0.25 & $0.16,0.24,0.4,0.8,2,4,8,12,16$ \\
& 1 & 0.2 & 0.04 & $0.5,1,3,10,20,30$ \\
& 1 & 0.1 & 0.01 & $0.6,6,10,20,40,60,100,200,400$ \\
\hline
\end{tabular}

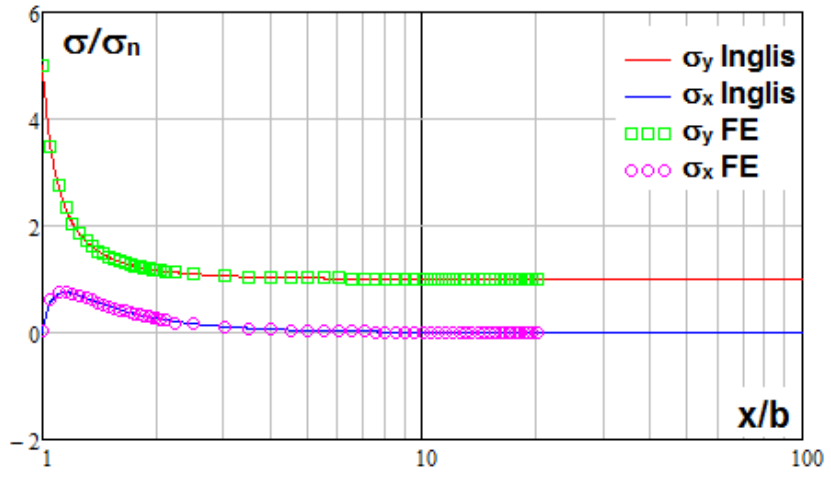

Figure 2.2 $-2 \mathrm{D}$ Stress distribution ahead of EH tip with apect ratio $t=b / a=$ $0.5, \rho / a=0.25$ : Inglis $[11$ analytical solution and $2 \mathrm{D} \mathrm{FE}$ results

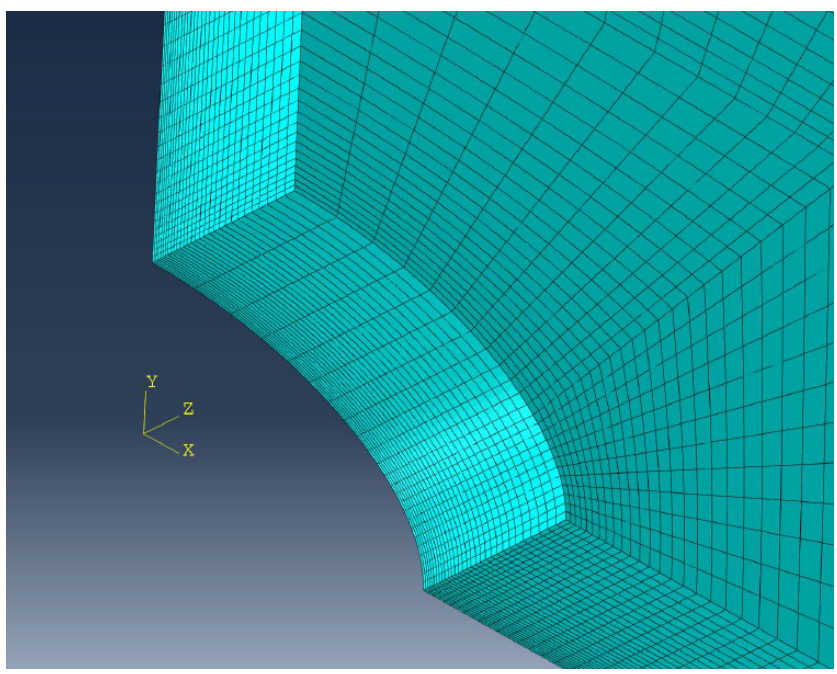

Figure 2.3 - 3D FE model of notched plate with $b / a=0.5$ and $B / \rho=4$. For SE cases, $1 / 4$ symmetry (with respect to planse $x y$ and $x z$ ); for EH cases, $1 / 8$ symmetry $(x y, x z$ and $y z))$. 


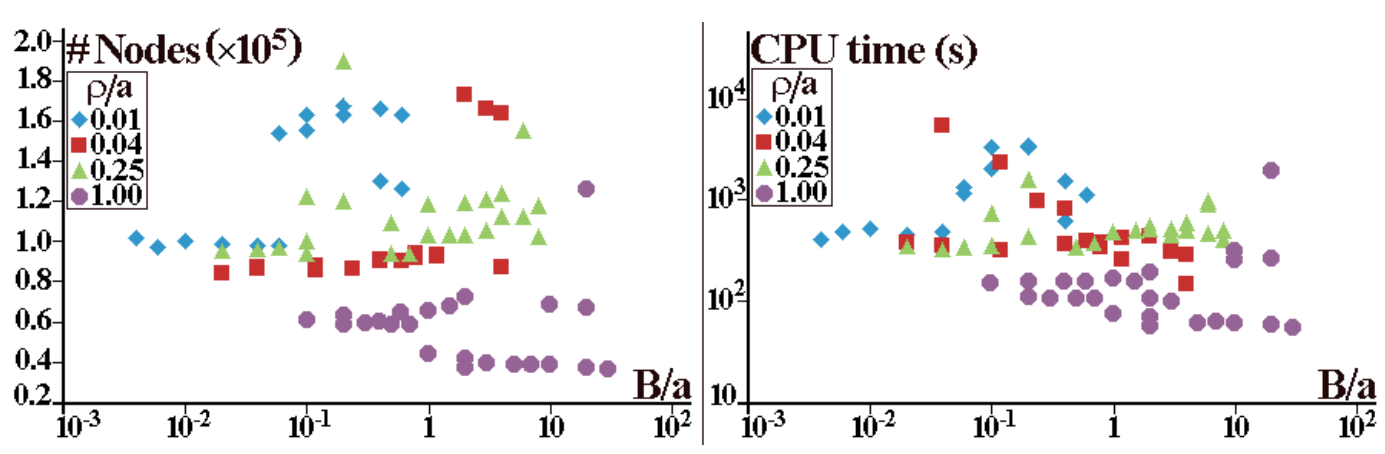

Figure 2.4 - (a) Number of nodes used in SE and EH notch analyses and (b) $\mathrm{CPU}$ time demanded for analysis

\section{2 .}

\section{D crack FE models}

Several LE FE 3D analyses were performed in Abaqus $[6]$ to evaluate the influence of thickness to crack size $B / a$ ratios on the crack tip fields of large SE(T)-like cracked plates. The software routines of submodeling were used to reduce the computational cost without compromising the accuracy of the resulting stress and strain distributions around their crack tips.

\subsection{1.}

\section{Submodeling technique}

Submodeling is very useful for local analyses, when the detailed modeling of only a small part of a model is required, and when this partially detailed modeling does not affect the overall solution of the global model. They are helpful e.g. when dense refinements are needed only in a specific region of the model, such as in crack problems, or else in localized plasticity analyses, where a LE global model can be used to generate the loads for a more complex elastic-plastic sub model.

A Submodeling analysis includes two basic steps. First, the solution of the relatively simple global model is obtained, without detailing the region around the critical point. Then, a second submodel is built for that critical region, containing all the details needed for the local analysis (e.g. dense refinements, non-linear material characteristics...). The nodes of the submodel boundary are loaded by the properly interpolated stress and/or displacement fields generated by the global model at those points. Finally, the local analysis is run with a refined mesh or other features appropriate for the local analysis purposes.

The adequacy of the submodeling technique is case-dependent, since it does not automatically update global analyses. However, it is particularly useful for numerical analyses of lightly loaded cracked structures within the 


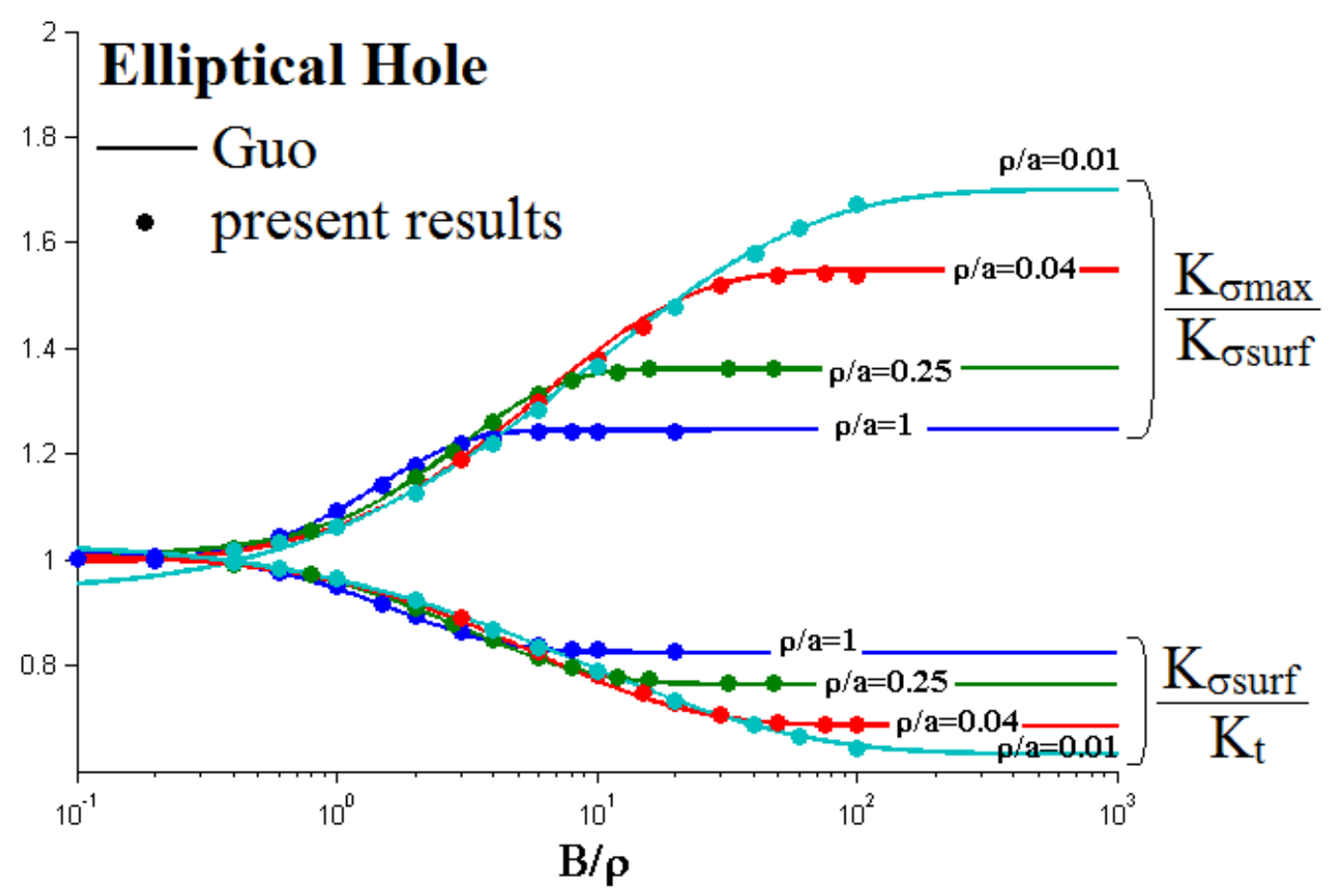

Figure 2.5 $-K_{\sigma_{\max }} / K_{\sigma_{\text {surf }}}$ and $K_{\sigma_{\text {surf }}} / K_{t}$ VS $B / \rho$ for EH: comparison of present results and those obtained by She and Guo, given by eq. 2.1 to 2.2

scope of this work. Indeed, globally LE structures ignore the influence of crack tips in regions far enough from them, whereas local features, such as the $3 \mathrm{D}$ effects studied in this work, are important only near those tips [18,19]. Indeed, the transversal restriction which is important near crack tips, due to the high stress gradients that act there, may be negligible far from it, as in the case of the large plate studied here, which works under pl- $\sigma$ conditions far form its crack tip. Therefore, the use of 3D FE in this case is really required only in a limited region around the crack tip, an ideal situation for sub models.

Moreover, besides being numerically efficient, the submodeling technique used here also has some non-negligible advantages over the Boundary Layer approach [18, 19, 22]. Its crack tip fields are calculated considering all the load characteristics, since they are not restricted to $K$-hypothesis limitations. Hence, it recognizes e.g. the nominal stress effects far from the crack tip, which are intrinsically ignored when $K$-field loading conditions are assumed to be valid. Finally, it allows the examination of cracks with high $B / a$ ratios, thus of relatively shallow cracks and thick plates.

\subsection{2.}

\section{D global model}

The global model was built with CPS6 and CPS8 elements from Abaqus [6] library. CPS6 and CPS8 are respectively 6-node and 8-node quadratic pl- $\sigma$ 


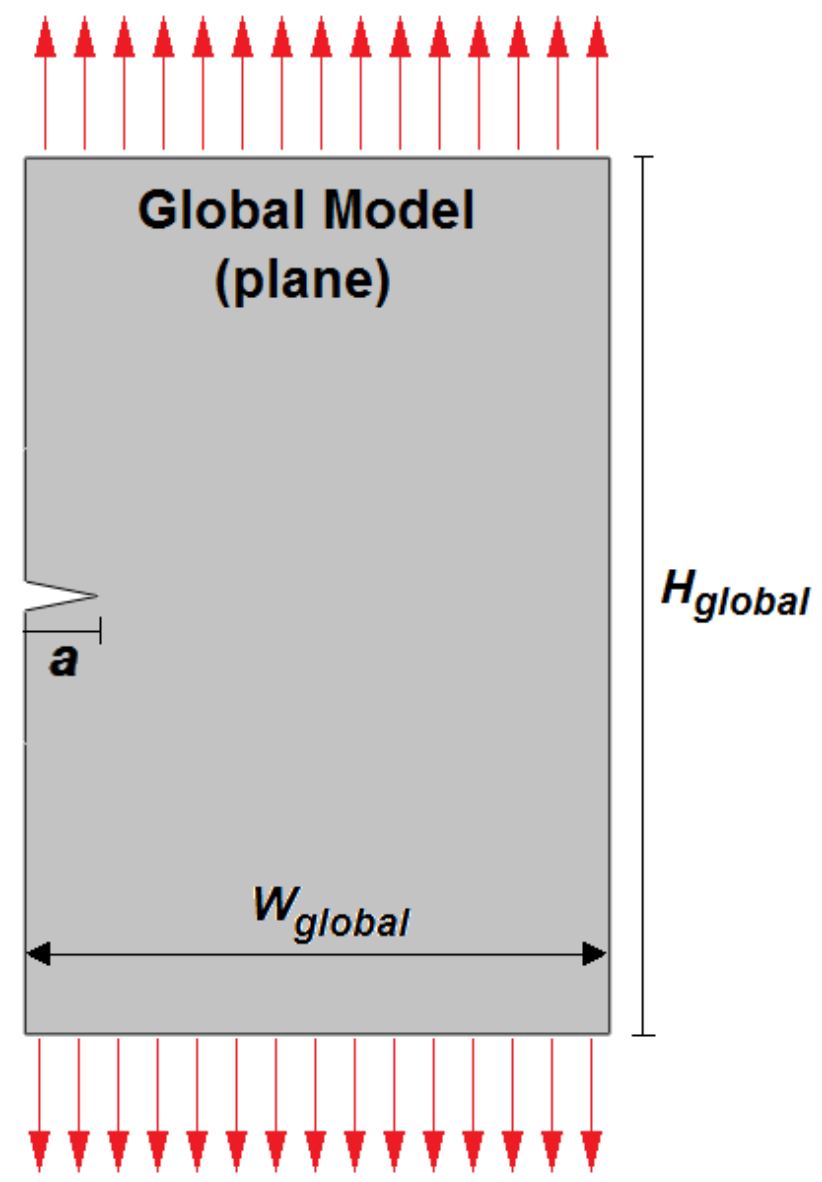

Figure 2.6 - Submodeling technique applied in the evaluation of 3D effects close to crack tips in globally pl- $\sigma$ loaded specimen

element, with two degrees of freedom per node. The crack tip was modeled with collapsed quadrilateral quadratical quarter point elements, able to reproduce the $1 / \sqrt{r}$ singularity, as described elsewhere [1,9]. Such elements are originaly quadrilateral 8-nodes elements, but the nodes of a face are made coincident and remain so during the analysis.

In order to approximate the semi-infinite cracked plate, avoiding boundary influence, the global model was built with width and height ( $W_{\text {global }}$ and $\left.H_{\text {global }}\right)$ equal to $1000 a$.

Since the global model would be run only once and feed submodels with different sizes, a very refined mesh was used. 36013 elements and 11960 nodes were used in the mesh construction, with sizes smoothly decreasing from $100 a$ at the model far boundaries to $a / 1000$ at the crack tip (see figures 2.7 and 2.8. 

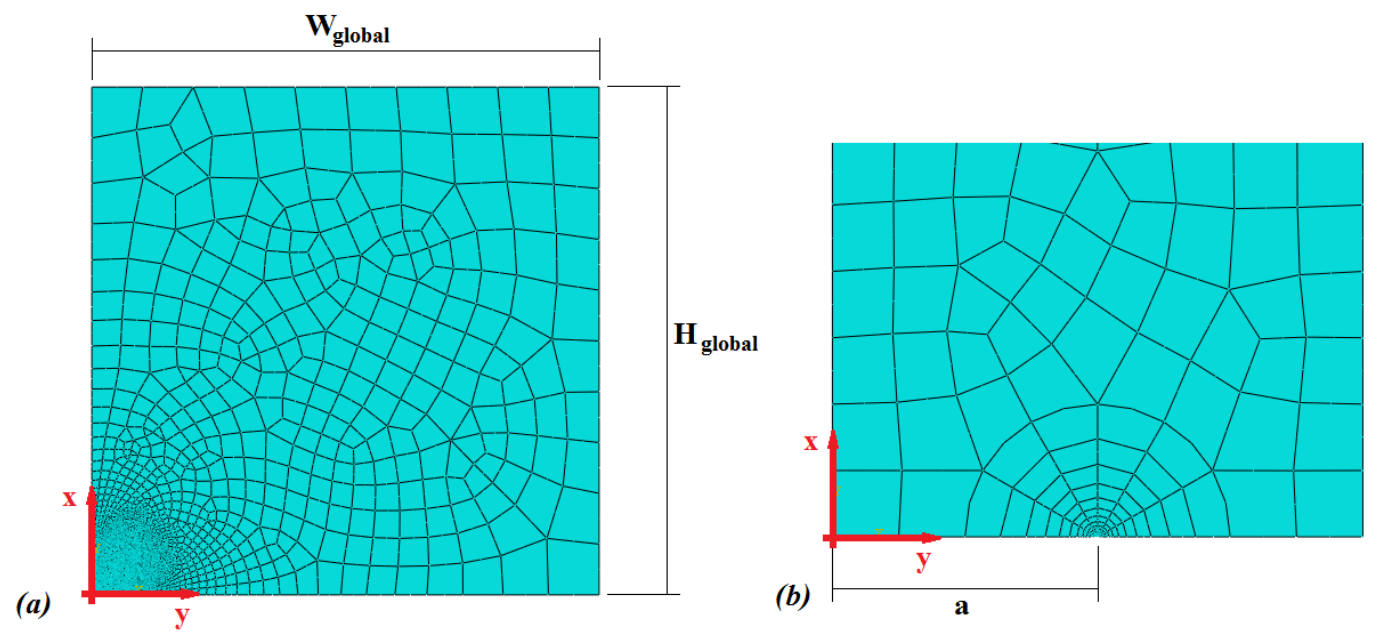

Figure 2.7 - (a) Global Model Mesh using pl- $\sigma$ elements and (b) detail of the mesh close to the crack tip

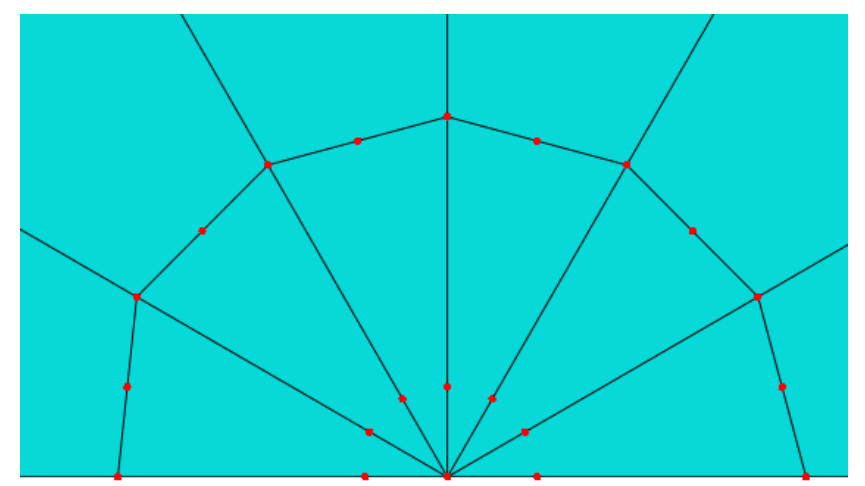

Figure 2.8 - Quarter Point Elements around crack tip

\subsection{3. 3D submodels}

The submodels were built covering a wide range (3 orders of magnitude) of $B / a$ ratios. Like the notch models described in section 2.1. C3D20 elements were used in the mesh construction. The submodels were built with height and width $H_{\text {sub }}=W_{\text {sub }} \geq 2.5 B$, which demonstrated to be enough for the $3 \mathrm{D}$ effects to fade (see section 3.2 .

The mesh was created using the sweep method, repeating a plane mesh in the free surface several times along the $z$-direction (thickness). A finer mesh (more divisions in $z$-direction) was used close to the free surface and to the crack tip, for reasons which will be illustrated. A bias ratio equal to 51, same as in [22], was used in this one-way refinement.

Collapsed 20-node quadratic brick quarter-point elements [9, 12] were created along the crack front. 


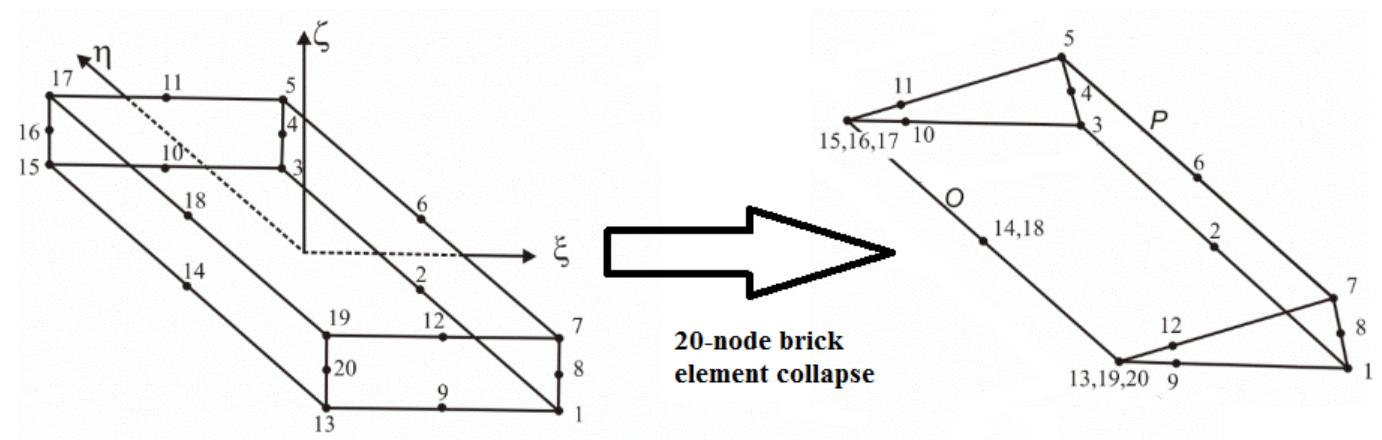

Figure 2.9 - Collapse of a 20-node brick element into a prismatic element (source: 12])

Such collapsed elements are created from regular 20-node brick elements by collapsing an entire face of the element, so that some of the nodes become coincident (see figure 2.9). These nodes remain coupled during the analysis.

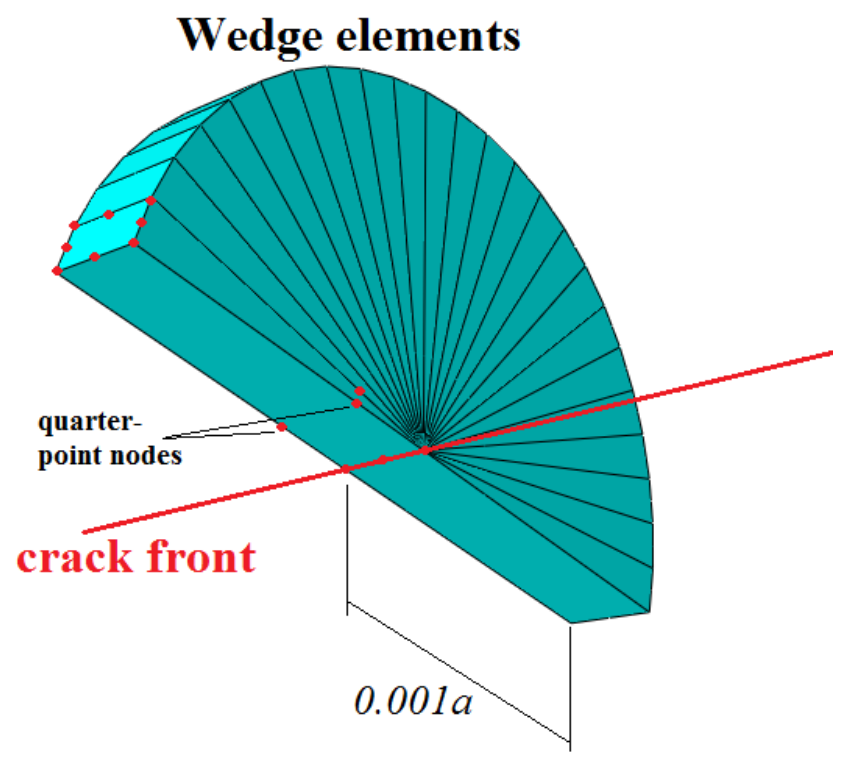

Figure 2.10 - Quarter-point collapsed brick elements around the crack front

Figures 2.10, 2.11 and 2.12 show an example of a mesh in a submodel with $B / a=0.6$.

The SIF $K_{I}$ was calculated in Abaqus [6] for every node along the crack front, using a built-in $J$-integral routine and the correlation:

$$
\begin{aligned}
J & =\frac{1}{\bar{E}} K_{I}^{2} \\
\bar{E} & =E /\left(1-\nu^{2}\right)
\end{aligned}
$$

A refinement study was performed in order to achieve an adequate mesh able to capture the 3D effects close to the crack front. The case of 


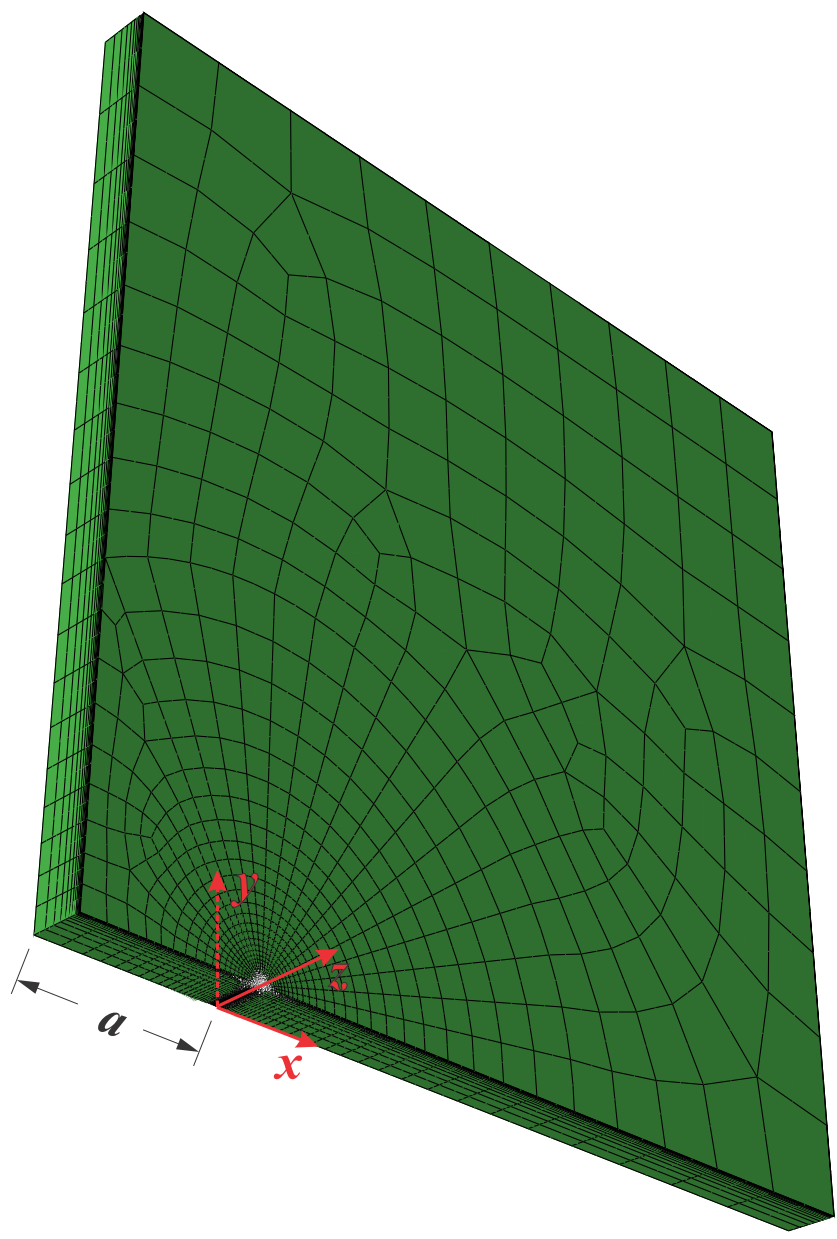

Figure 2.11 - Mesh of a submodel with $B / a=0.6$

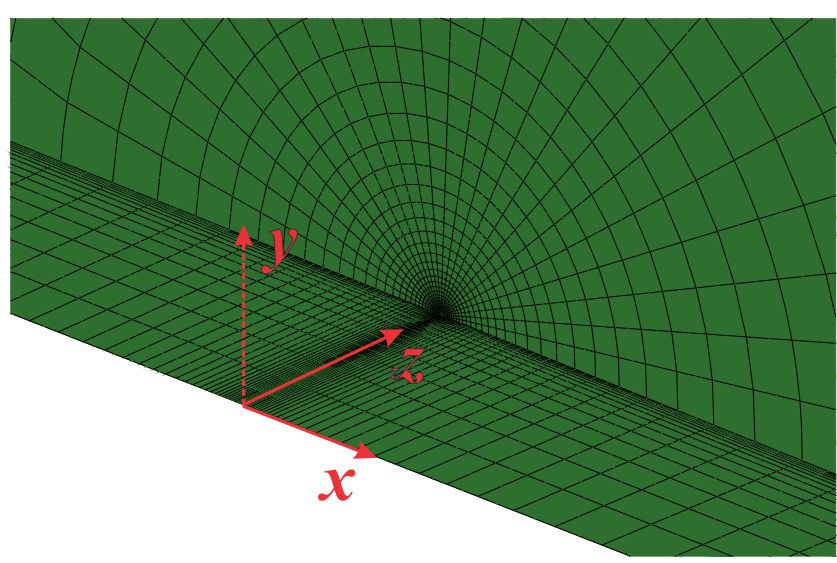

Figure 2.12 - Mesh of a submodel with $B / a=0.6$ close to the crack tip 
$B / a=10$ is here presented as an example. A starting relatively coarse mesh was progressively refined, and SIF and $\sigma_{y_{\mathrm{mp}}}$ were monitored for the several analyses. Two parameters were taken as representative of the mesh refinement: the number of divisions used in $z$-direction (thickness) and the size of the cracktip elements $\left(l_{\text {tip }}\right)$.

Figure 2.13 shows the $K_{I} / K_{I_{2 D}}$ VS $z / B$ distribution along the plate thickness for several refinements in $z$-direction, while Figure 2.14 presents $K_{I_{\max }}, K_{I_{\mathrm{mp}}}$ and $K_{I_{\mathrm{surf}}} \mathrm{VS} z / B$ behavior with the increase of mesh refinement.

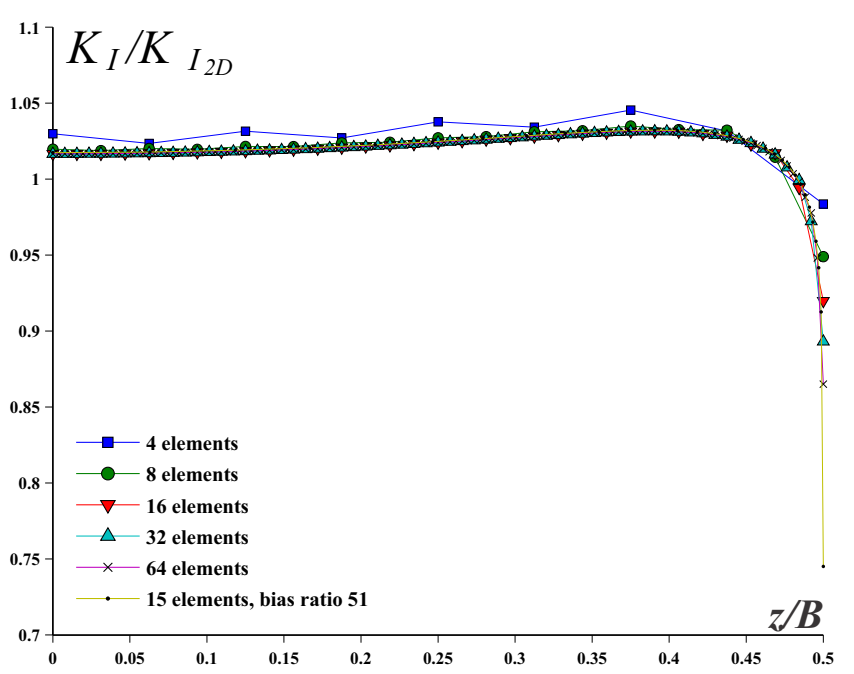

Figure 2.13 - Submodel $B / a=10$, convergence check: $K_{I} / K_{I_{2 D}}$ VS $z / B$ distribution along the crack front for several refinements along $z$-direction.

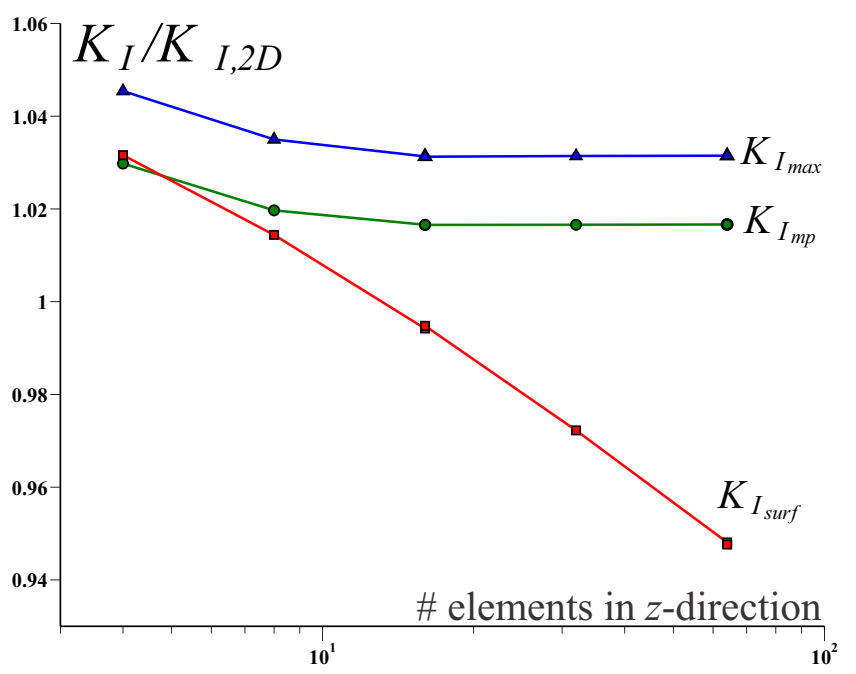

Figure 2.14 - Submodel $B / a=10$, convergence check: $K_{I_{\mathrm{mp}}}, K_{I_{\max }}$ and $K_{I_{\mathrm{surf}}}$ variation with the number of elements used in $z$-direction

As it can be seen in Figure 2.14, $K_{I_{\mathrm{mp}}}$ and $K_{I_{\max }}$ reach assymptotic limits for 16 elements along the thickness. In Figure 2.13 , the $K_{I} / K_{I_{2 D}}$ distributions can barely be distinguished along most of the thickness. 
The exception occurs in the free surface $(z / B=0.5)$ and its very close vicinities, where $K_{I}$ seems to diverge with the refinement. Such behavior is already expected from Bazant \& Estenssoro [2] results. As discussed in chapter 1.2, $K_{I}$ in the free surface should be zero, as the crack is under pure mode-I and its front is perpendicular to the free surface. She \& Guo [22] highlight the very same behaviour in their research on thin plates.

Besides, the SIF calculation in the used software is based on a correlation appropriate for pl- $\varepsilon$ condition, which cannot provide accurate results in the free surface, where the material is under pl- $\sigma$

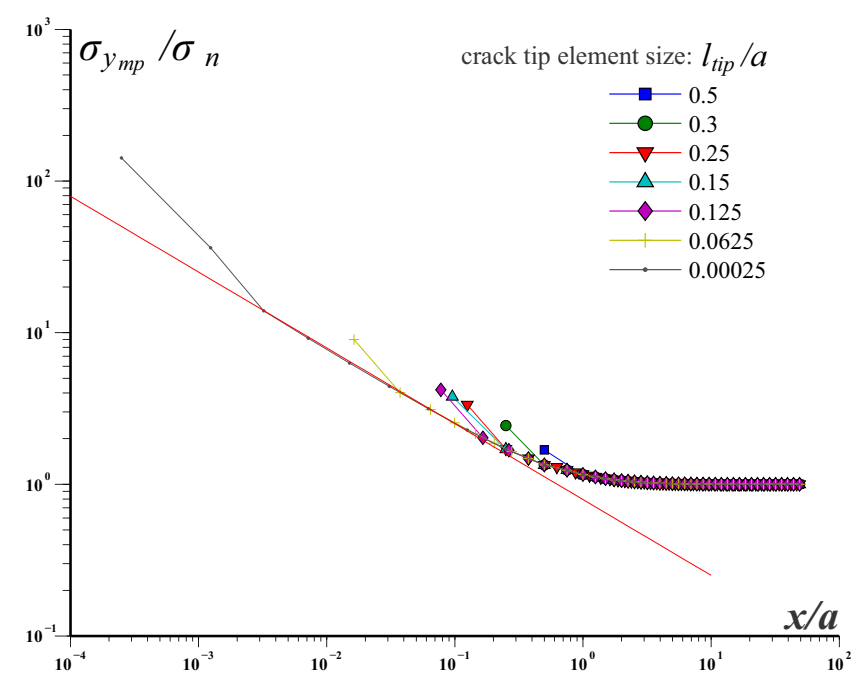

Figure 2.15 - Submodel $B / a=10$, convergence check: $\sigma_{y_{\mathrm{mp}}}$ gradient ahead of crack tip

Concerning $\sigma_{y_{\mathrm{mp}}}$ stress gradient ahead of the crack tip, Figure 2.15 shows that, the thinner the mesh, the closer the $\sigma_{y}$ profile gets to the $K$-solution close to the crack tip. Also, it shows that elements shorter than $0.125 a$ at the crack tip were necessary to capture the transition from the $K$-dominated region to the nominal stress $\sigma_{n}$-dominated region.

\section{3.}

\section{Crack growth model}

FRANC3D was used to simulate the growth of large and small edge cracks with an initially straight front in a large plate. This versatile Fracture Mechanics code is equipped with specific routines to track the local propagation of each point along a 3D crack front and to obtain the crack front shape of the next propagation step, as described elsewhere along this chapter. Within this work scope, the fatigue cracks are assumed to grow under LEFM conditions.

Since the solution of linear elastic problems is unique and proportional to the imposed load, the calculated SIF $K_{\text {calc }}$ along the crack front can be 
interpreted as a shape function. Therefore, the following equations apply to describe the various crack loading parameters:

$$
\begin{aligned}
K_{\max } & =K_{\text {calc }} \\
K_{\min } & =R \cdot K_{\text {calc }} \\
R & =\frac{K_{\min }}{K_{\max }} \\
\Delta K & =K_{\max }-K_{\min }
\end{aligned}
$$

Without any loss of generality, the analyses developed here considered $R=0$ and $P=1$, thus in the propagation cases described below:

$$
\Delta K=K_{\max }=K_{\text {calc }}
$$

It is assumed that the local crack advance at any specific point of the crack front follows the simple Paris' fatigue crack propagation rule. From the provided $K_{\text {calc }}$ values at the front nodes in the present step, a crack propagation vector $\overrightarrow{\delta a}$ is obtained, which can be described as the product of a local advance module $\Delta a$ and a unitary vector $\vec{p}$ determining the local crack propagation direction. The local direction vector $\vec{p}$ is fixed: it must be parallel to the crack plane ( $x z$ in this case) and normal to the crack front at each point, as the model is symmetric with respect to the $x z$ plane. The crack advance increments are assumed to follow Paris' rule, thus they can be described for any given node $i$ at every $j^{\text {th }}$ growth step in terms of a given advance parameter $\Delta a_{\text {mean }}$ by:

$$
\Delta a_{j i}=\Delta a_{j_{\text {mean }}}\left(\frac{\Delta K_{j i}}{\Delta K_{j_{\text {mean }}}}\right)
$$

Note that, as a result of the assumed hypotheses (LE conditions, constant amplitude loading, Paris' rule, no crack closure), the SIF distribution along the entire crack front $K(z)$, and therefore the ratios between the SIF increments at each crack front node and the mean SIF increment at each load step, $\Delta K_{j i} / \Delta K_{j_{\text {mean }}}$, are a function uniquely of $a_{i}(z)$, the crack length at each node in that step. Hence, $\Delta a_{j_{\text {mean }}}$ becomes an arbitrary analysis parameter, dissociated from the number of load cycles and everything else. Moreover, as $a_{i}(z)$ as well as $\Delta a_{j i}(z)$ are expected to vary along the crack front, the term crack length is not adequate in the strict sense, since the modeled cracks have several lengths along the plate thickness, as real cracks normally do. However, 
since the crack propagation problem in plate-like components is usually treated as if it was essentially $2 \mathrm{D}$, their cracks are normally described by a single overall length, in spite of their fronts being in fact slightly curved. Therefore, in the present case the length value at the plate free surface, $a_{\text {surf }}$, is adopted as a descriptive parameter of the crack overall length. This specific value would be the one measured by optical methods, or by any other surface-dependent technique for measuring crack length, such as crack gages, e.g.

After solving each particular crack propagation step, the obtained crack front increment is smoothed and fitted by a $7^{\text {th }}$ degree polynomial, in order to minimize the unavoidable numerical noise associated with the $K_{I}(z)$ solution. Such a high order polynomium was chosen in order to capture and describe the effects of odd $K_{I}$ distributions in relatively shallow cracks.

The simulated edge-cracked plates are built with the same overall dimensions $H, B$, and $W$. The initial cracks are introduced in one of their edges all with straight fronts, but with different depths $a_{0}$. The plate models assume symmetric boundary condition at the plate mid-plane $z=0$ and are supposed tensioned by a unitary uniformly distributed load at their upper and lower boundaries.

In the mesh construction, quarter-point quadratic wedge elements from Abaqus [6] library are used to model the crack front, a default procedure in FRANC3D software. The SIF $K_{I}$ is internally calculated by FRANC3D from Abaqus' results, using an in-built $J$-integral routine.

Figure 2.16(a) shows the overall model used in these simulations and Figure 2.16(b) illustrates two possible curved crack fronts after the initially straight crack propagates for a while. Table 2.5 presents the parameters used in the various models:

Table 2.5 - Crack growth model parameters

\begin{tabular}{|l|c|l|}
\hline Parameter & Symbol & Value \\
\hline Poissons ratio & $\nu$ & 0.3 \\
Young's modulus & $E$ & $200 \mathrm{GPa}$ \\
Thickness & $\mathrm{B}$ & $5 \mathrm{~mm}$ \\
Width & $W$ & $4 B$ \\
Height & $H$ & $2.5 B$ \\
Crack initial length & $a_{0}$ & $0.02 B, 0.2 B, B$ \\
Paris rule exponent & $n$ & $2.0,4.0$ \\
\hline
\end{tabular}



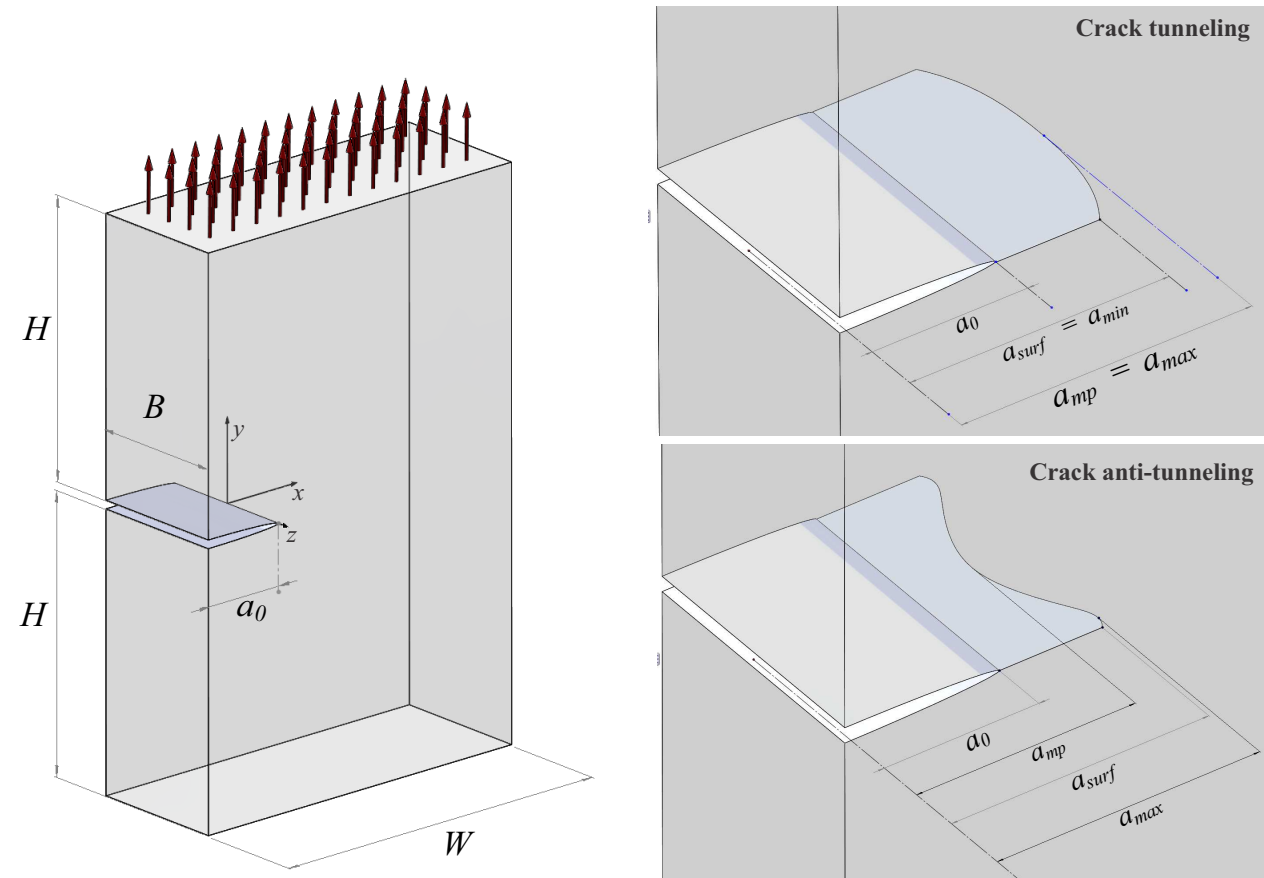

2.16(a): Plate under uni-axial load with 2.16(b): Crack tunneling and antiinitially straight crack with length $a_{0}$ tunneling: length measurement at different points of the crack front

Figure 2.16 - Crack propagation model 


\section{3 \\ Results}

This chapter presents the results obtained with the models described in chapter 2 .

\section{1.}

\section{D effects on notch tip fields}

Figure 3.1 shows the stress and strain concentration factors $K_{\sigma}$ and $K_{\varepsilon}$ distributions at the notch tip along the plate thickness for an elliptical hole with $t=b / a=0.5$ and $\rho / a=0.25$, to illustrate the typical results obtained from such analyses. Note in particular that $K_{\sigma} \neq K_{\varepsilon}$ and that for relatively thick plates (high $B / a$ ratios) their maximum values occur close to the plate surface $(z / B$ close to 0.5$)$, whereas for thinner plates such values occur at the plate center $(z / B=0)$. These results corroborate Yang's observation that $K_{\sigma}$ and $K_{\varepsilon}$ are different along the notch tip even in the LE case [30].

Figure 3.2 shows how the stress and strain concentration factors $K_{\sigma_{\max }}$ and $K_{\varepsilon_{\max }}$ behave as a function of the plate thickness-to-notch tip radius ratio $B / \rho$. For relatively thin plates or blunt notches (low $B / \rho$ ratios), the maximal stress will occur in the plate middle plane. For thicker plates and sharper notches, both $K_{\sigma_{\max }}$ and $K_{\varepsilon_{\max }}$ dislocate towards the free surface. Note how different such values can be (up to $15 \%$ for the analyzed models) and how their positions are slightly dissociated. Note also that $K_{\sigma_{\max }}$ can be up to about $8 \%$ higher than the $2 \mathrm{D} \mathrm{SCF} K_{t}$. But, more importantly, note that it also shows that $K_{t}$ measurements made (as usual) in the free surface may severely underestimate the maximal stress that occurs at the notch root, up to almost 25\%. This result indicates that 3D effects on the stress and strain fields along notch roots may indeed be non-negligible for some practical applications.

Figure 3.4 shows how $K_{\sigma_{\max }}, K_{\sigma_{\mathrm{mp}}}$, and $K_{\sigma_{\text {surf }}}$ vary with $B / \rho$ for the elliptical hole. It synthesizes much of what has been recently published in the literature. It reinforces that the error in $K_{\sigma_{\max }}$ predictions based on $2 \mathrm{D}$ models can be up to about $8 \%$. 


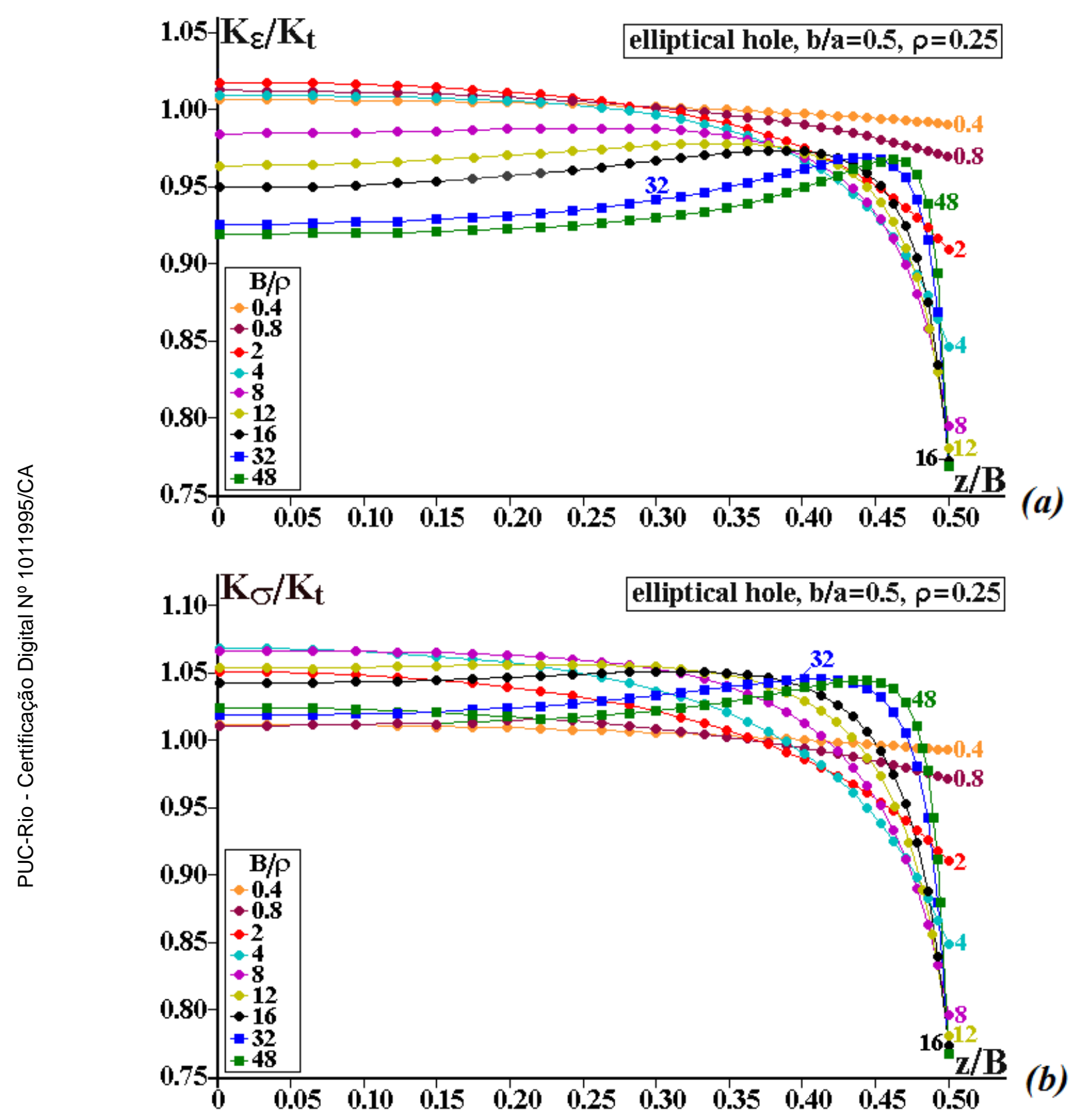

Figure $3.1-K_{\varepsilon} / K_{t}$ (a) and $K_{\sigma} / K_{t}$ (b) along $z / B$ for an Elliptic Hole with $b / a=0.5, \rho / a=0.25$ 


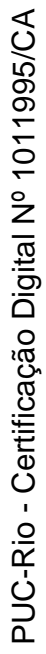

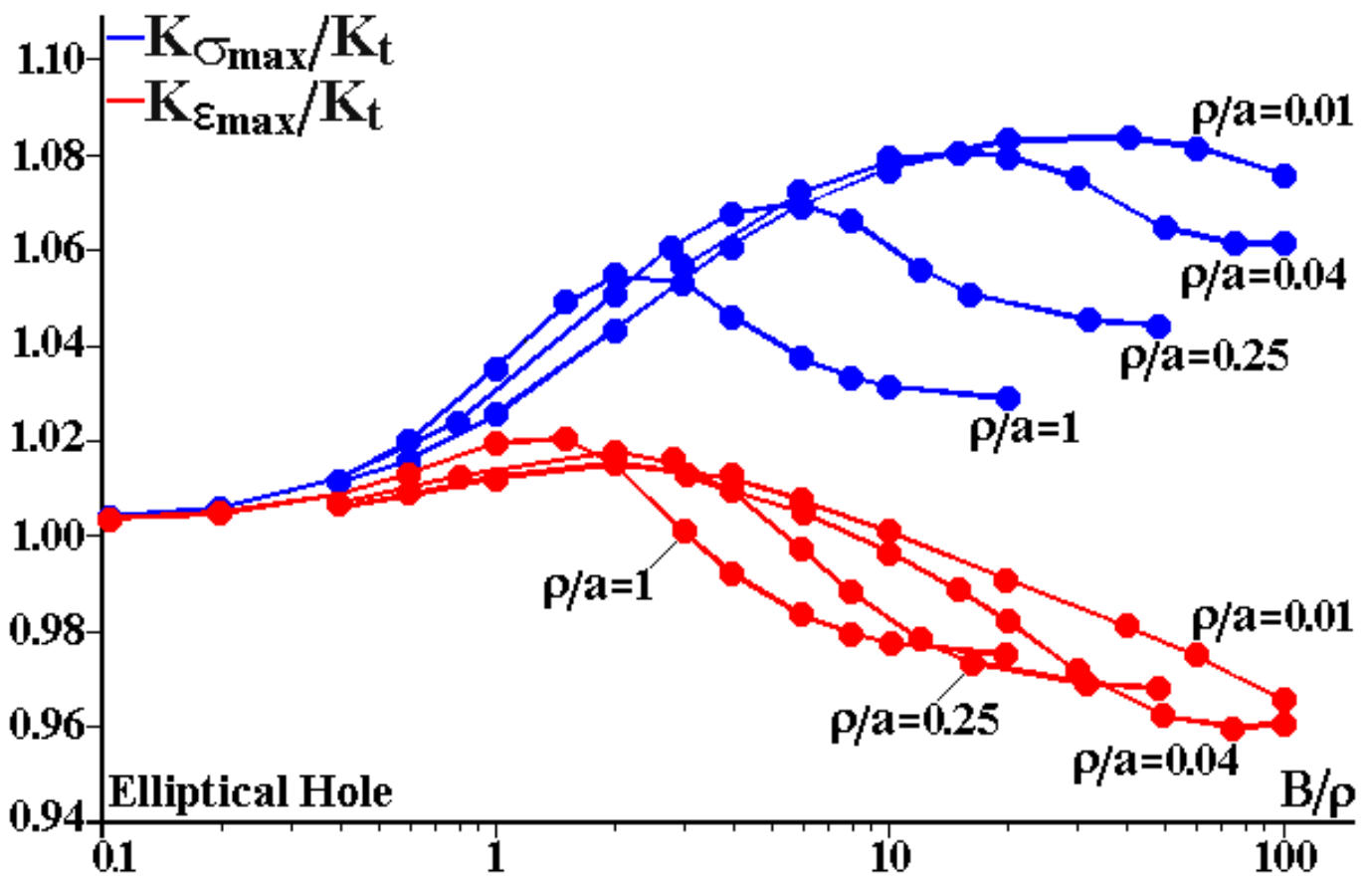

Figure 3.2 $-K_{\sigma_{\max }} / K_{t}$ and $K_{\varepsilon_{\max }} / K_{t}$ variation with $B / \rho$ for elliptical holes

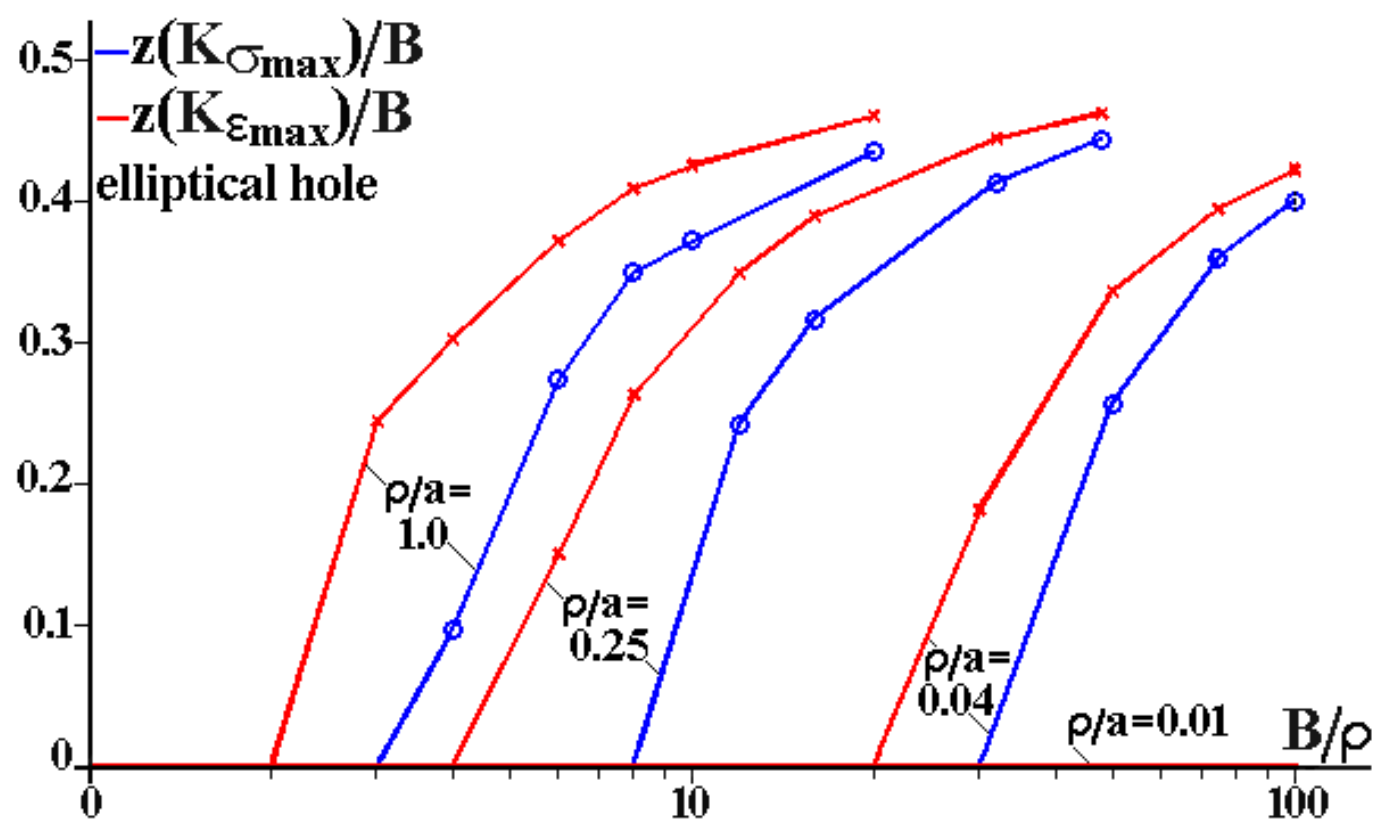

Figure 3.3 $-z / B$ Position of $K_{\varepsilon_{\max }} / K_{t}$ and $K_{\sigma_{\max }} / K_{t}$ for different $B / a$ values 


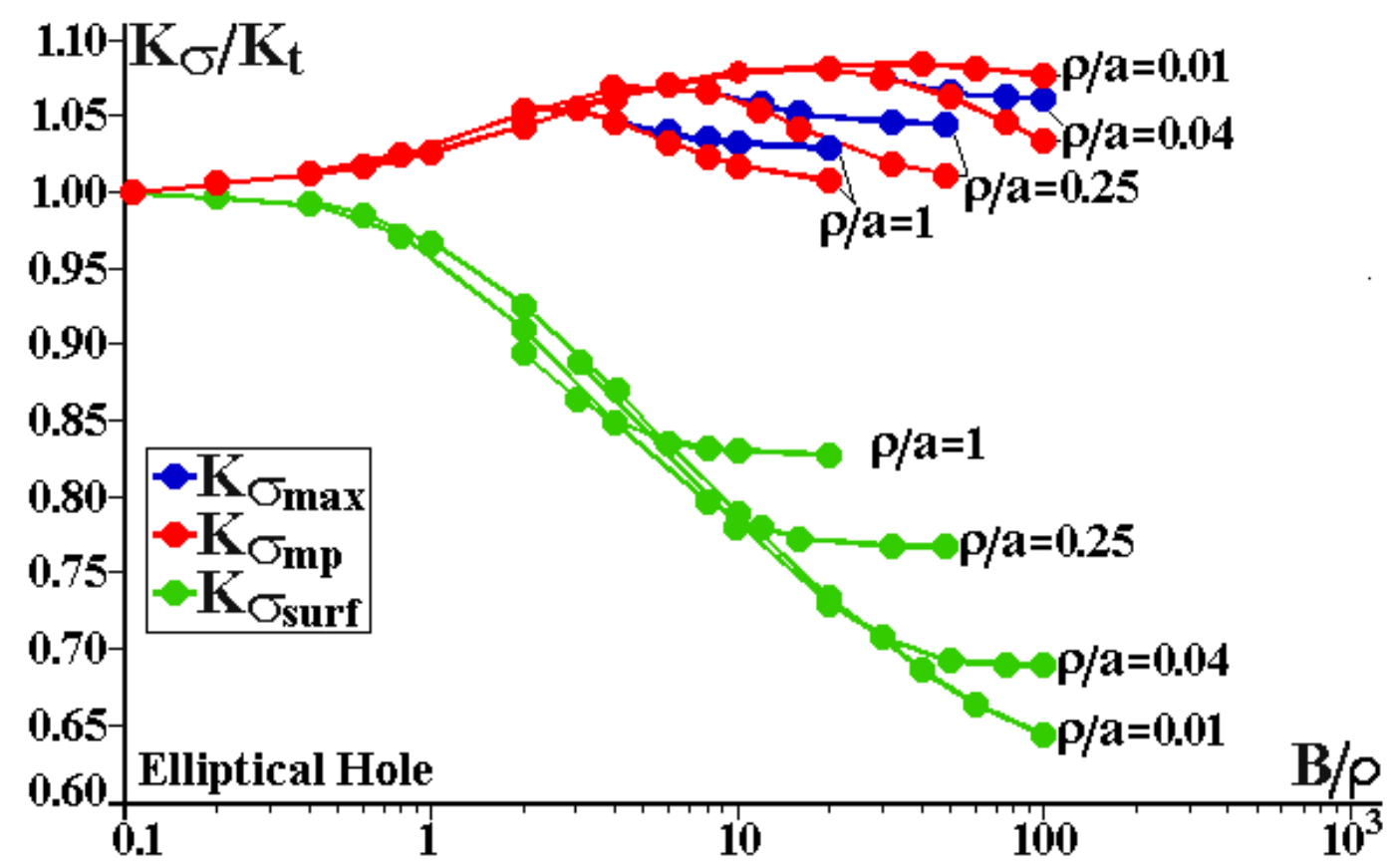

Figure 3.4 - Variation of $K_{\sigma_{\max }} / K_{t}, K_{\sigma_{\mathrm{mp}}} / K_{t}$, and $K_{\sigma_{\text {surf }}} / K_{t}$ with the thickness to root radius ratio $B / \rho$ for the elliptical holes

Figure 3.5 shows how the out-of-plane constraint factor $T_{z 0}$ varies along the tip of semi-elliptical notches in relation to its maximum value at the notch center, $T_{z 0_{\mathrm{mp}}}$. As expected, $T_{z 0}$ is maximal at the mid-plane and zero at the free surface. $T_{z 0}$ increases monotonically from the free surface inwards, and the thicker the plate is, the closer to the plate face it reaches the maximal value $T_{z 0_{\mathrm{mp}}}$.

The behavior of the transversal constraint $T_{z 0_{\mathrm{mp}}}$ at the middle point of the notch tip is illustrated in Figure 3.6, normalized by Poisson's ratio $\nu$. Note that the ratio $T_{z 0_{\mathrm{mp}}} / \nu$ increases with $B / \rho$, but not indefinitely. It grows from 0 , in very thin plates $(B / \rho \leq 0.1)$, and asymptotically reaches a saturated value, characteristic of the $\rho / a$ value, which quantifies how sharp the notch is. This means that, as expected, very thin notched plates are dominated by a pl- $\sigma$ limit condition around the notch tip. As the plate relative thickness $B / \rho$ increases, the ratio $T_{z 0_{\mathrm{mp}}} / \nu$ also increases, but its saturated value is always smaller than 1. In other words, no matter how sharp the notch is, it is never able to provide enough transversal constraint for achieving pl- $\varepsilon$ limit conditions around its tip. This limit state can only be achieved around ideal crack tips, as studied further on. Nonetheless, figures 3.5 and 3.6 clearly show how the transversal constraint tends toward the pl- $\varepsilon$ limit case $T_{z 0}=\nu$ as both $B / \rho$ and $a / \rho \rightarrow \infty$, when the elliptical notches became idealized LE cracks, which are discussed in detail in section 3.2 .

Figure 3.7 shows the behavior of the main normal stress $\sigma_{y_{\mathrm{mp}}}$ gradient 


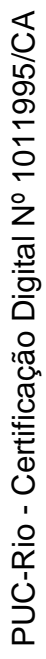

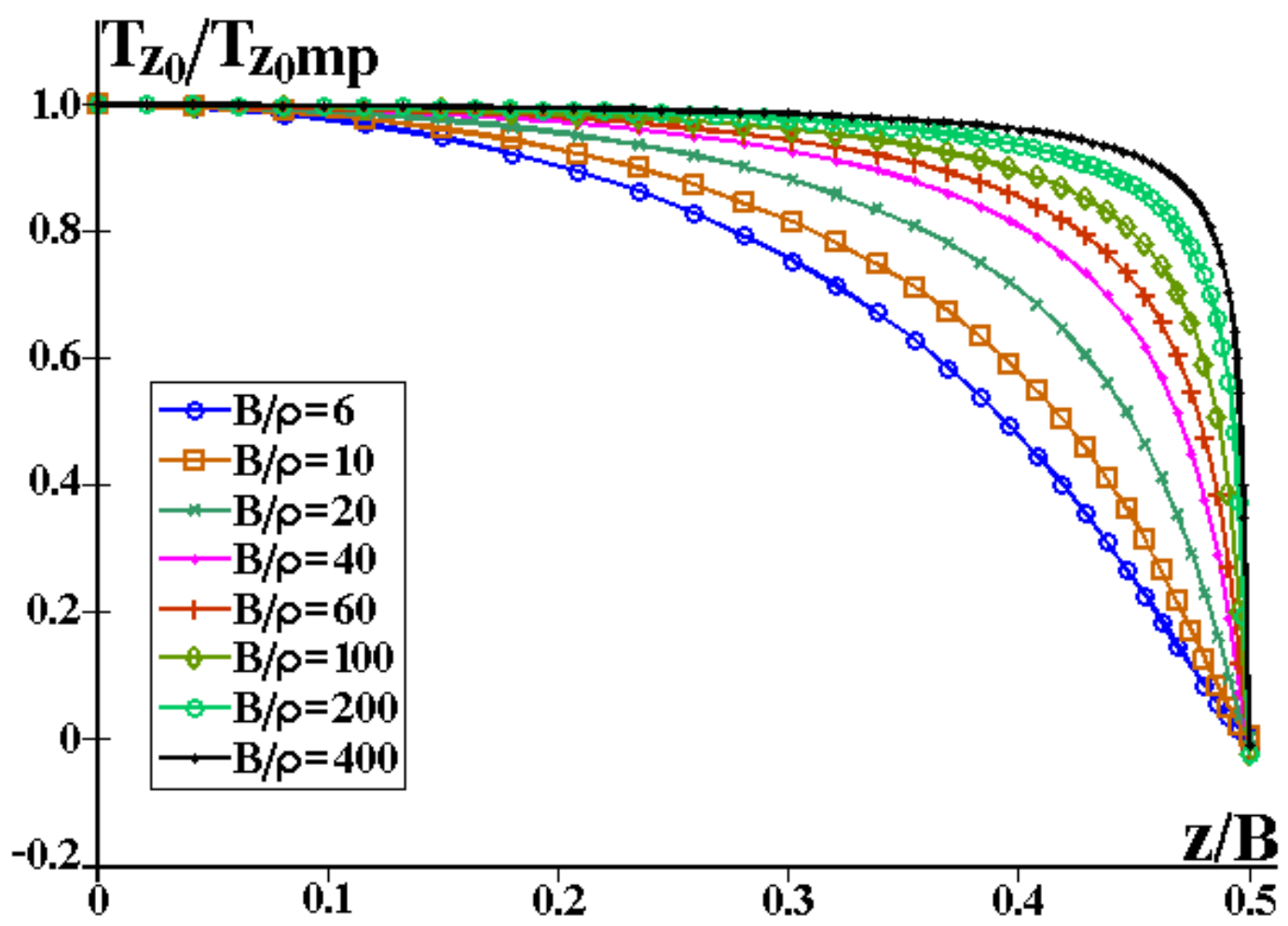

Figure $3.5-T_{z 0} / T_{z 0_{\mathrm{mp}}}$ vs. $z / B$ for the semi-elliptical notch with $\rho / a=0.01$

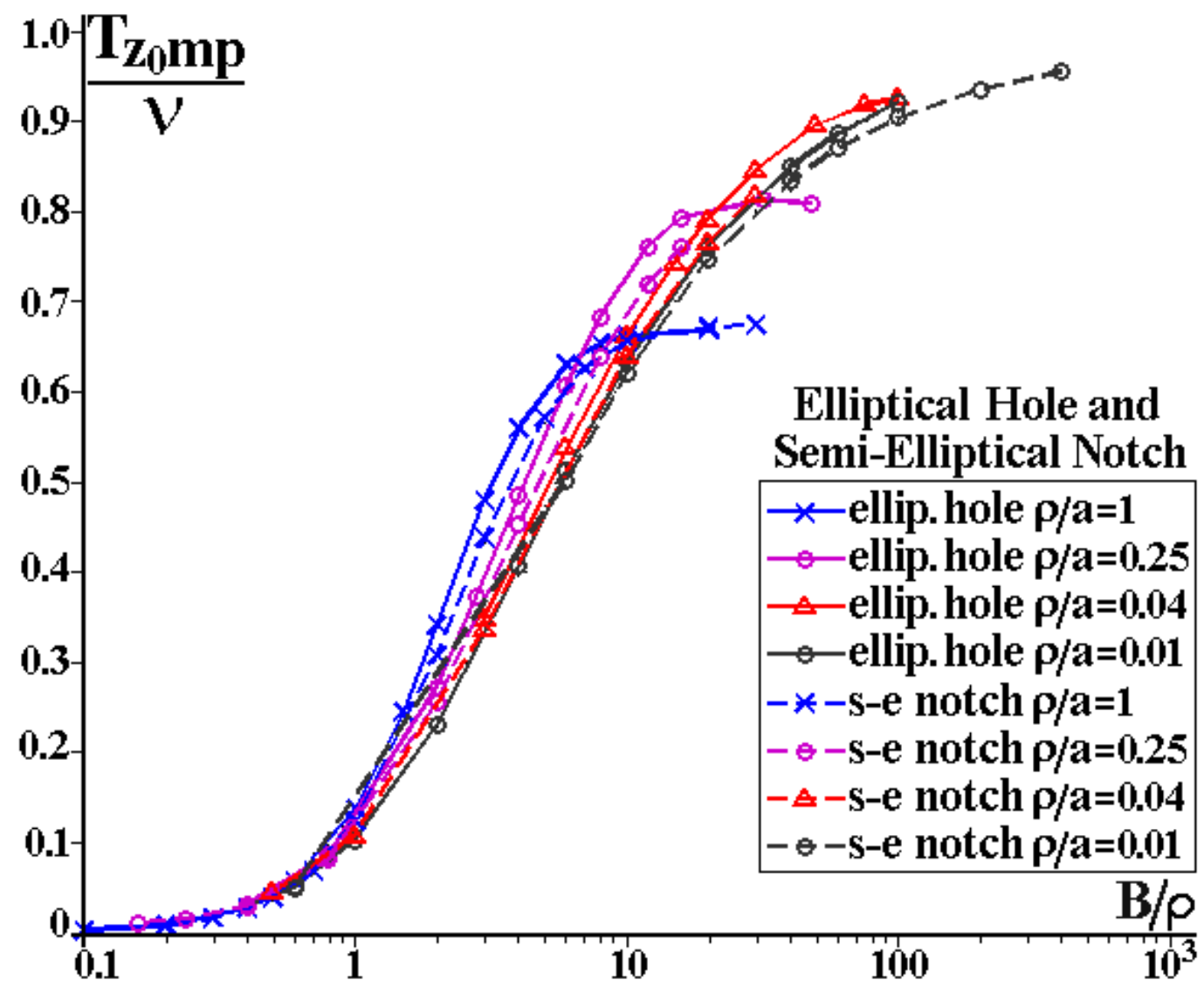

Figure 3.6 $-T_{z 0_{\mathrm{mp}}} / \nu$ versus $B / \rho$ for elliptical and semi-elliptical notches 
at the middle of the plate ahead of the notch tip, normalized by the stress at the notch tip $\sigma_{y 0_{\mathrm{mp}}}$. As already mentioned in the introduction, both Kirsh and Creager-Paris solutions (eq. 1.3 and 1.9 ) give good approximations for the $\sigma_{y} / \sigma_{y 0}$ ratio.

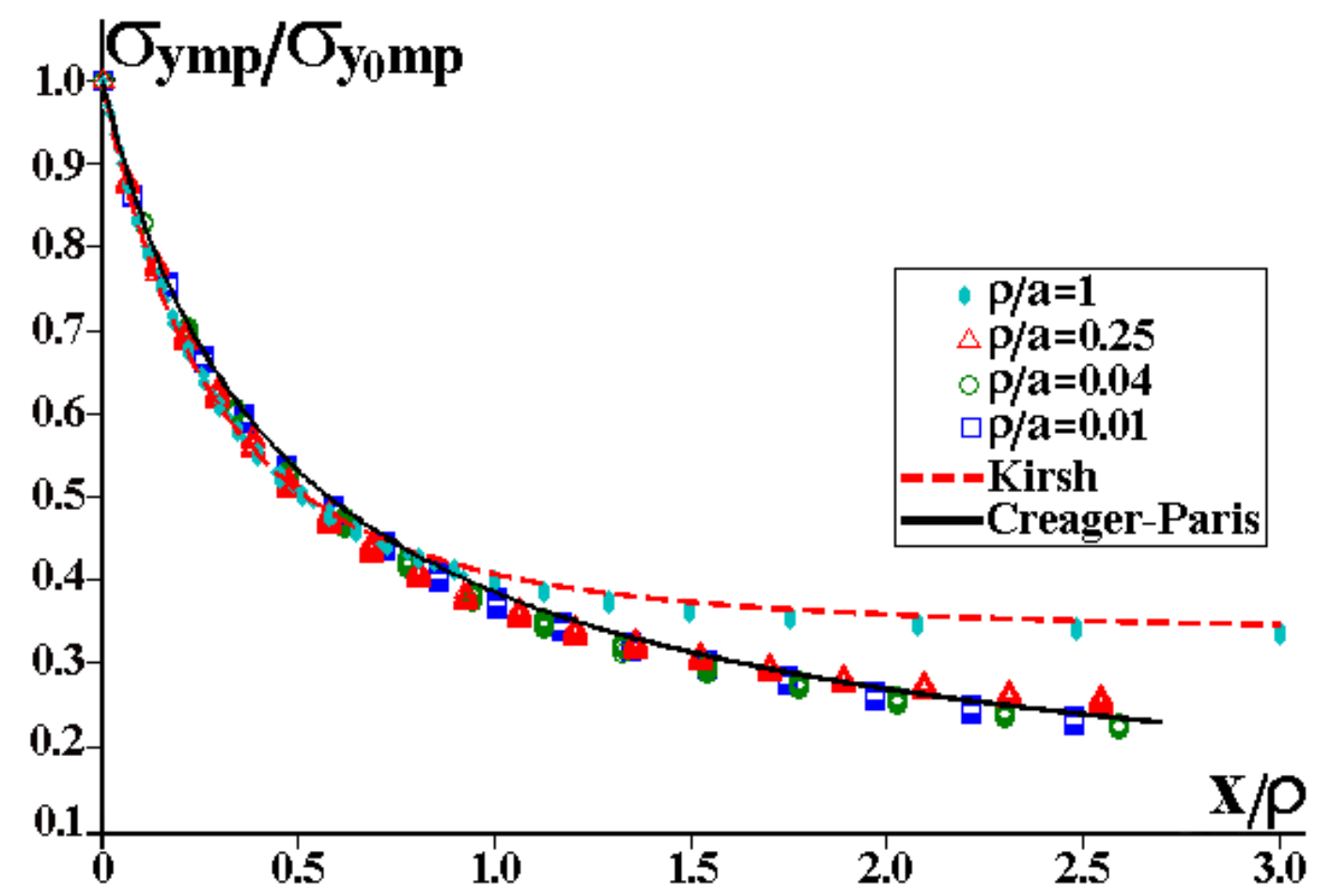

Figure 3.7 - Normalized gradient $\sigma_{y_{\mathrm{mp}}} / \sigma_{y 0_{\mathrm{mp}}}$ vs $x / \rho$ for several notches with different $\rho / a$ ratios

Figure 3.8 illustrates how the $\sigma_{y} / \sigma_{y 0}$ ratio varies ahead of the notch tip for several $z / B$ positions along the notch front, for a particular elliptical hole with $\rho / a=0.25$ and $B / \rho=12$. These results confirm that the $\sigma_{y} / \sigma_{y 0}$ gradient is almost independent from the thickness-to-notch root radius $B / \rho$ ratio and from the position $z / B$ along the plate thickness, as mentioned in section 1.1 .

The maxima stress and strain position indicates the location for crack initiation, whereas the stress gradients ahead of such critical points affect how a short crack propagates from the notch tip. According to the results presented above, if the cracks do prefer to start at maxima stress and strain points, as usually assumed in most damage models, they should do so in the center of the thinner notched plates $(z / B=0)$ and closer to the free surfaces $(z / B=0.5)$ of the thicker ones. But the growth of such initially small surface cracks is strongly dependent on the stress gradient around the notch tip, as discussed elsewhere $7,17,28$. Since the studied notches have much stronger stress gradients in $x$ than in $z$-direction, the short crack driving force decrease is sharper ahead than along the notch tip direction. Therefore, it may be argued that cracks initiated at a notch tip should prefer to advance first 


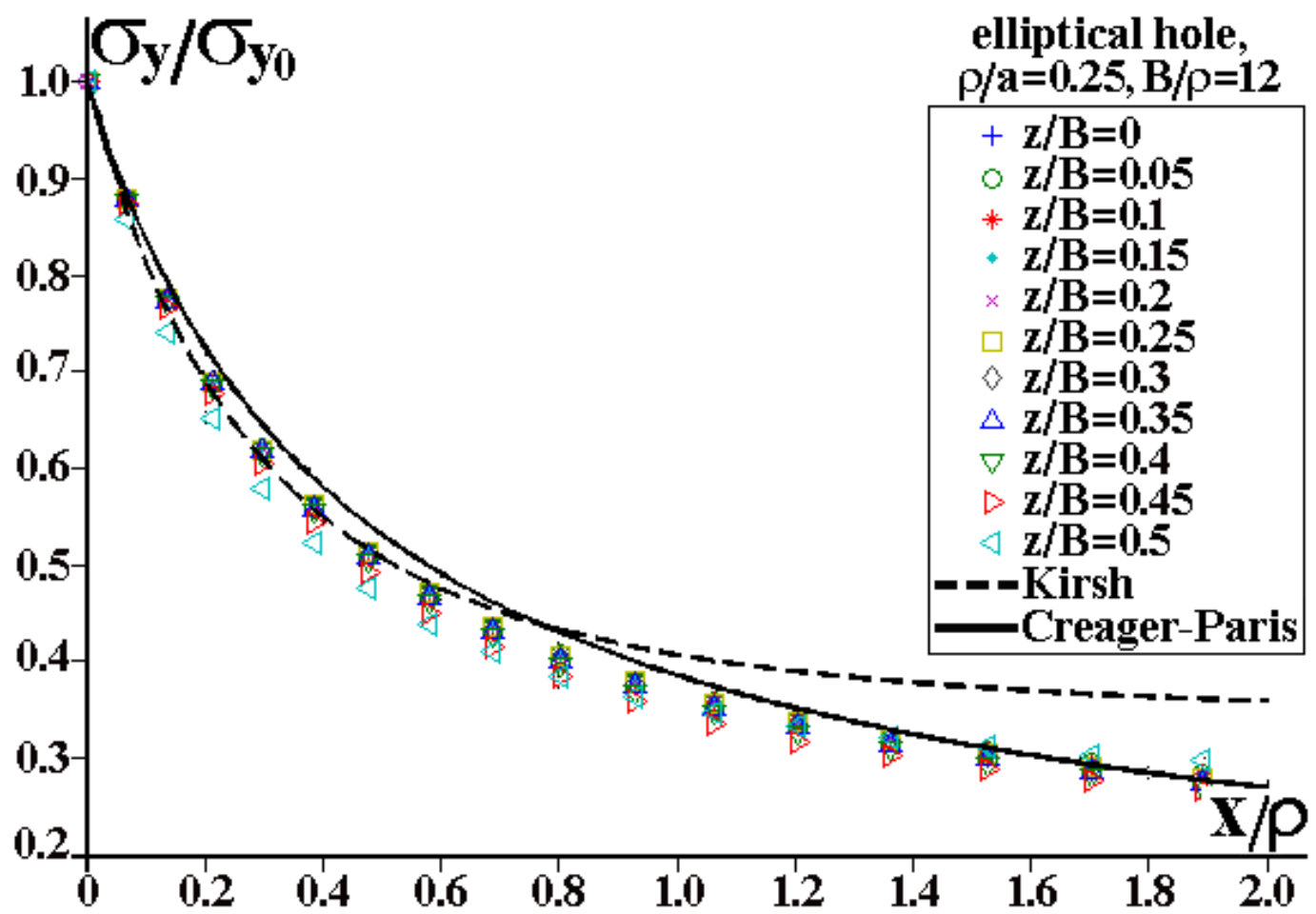

Figure $3.8-\sigma_{y} / \sigma_{y 0}$ vs. $x / \rho$ for an elliptical hole with $\rho / a=0.25$ and $B / a=12$ at different $z / B$ positions

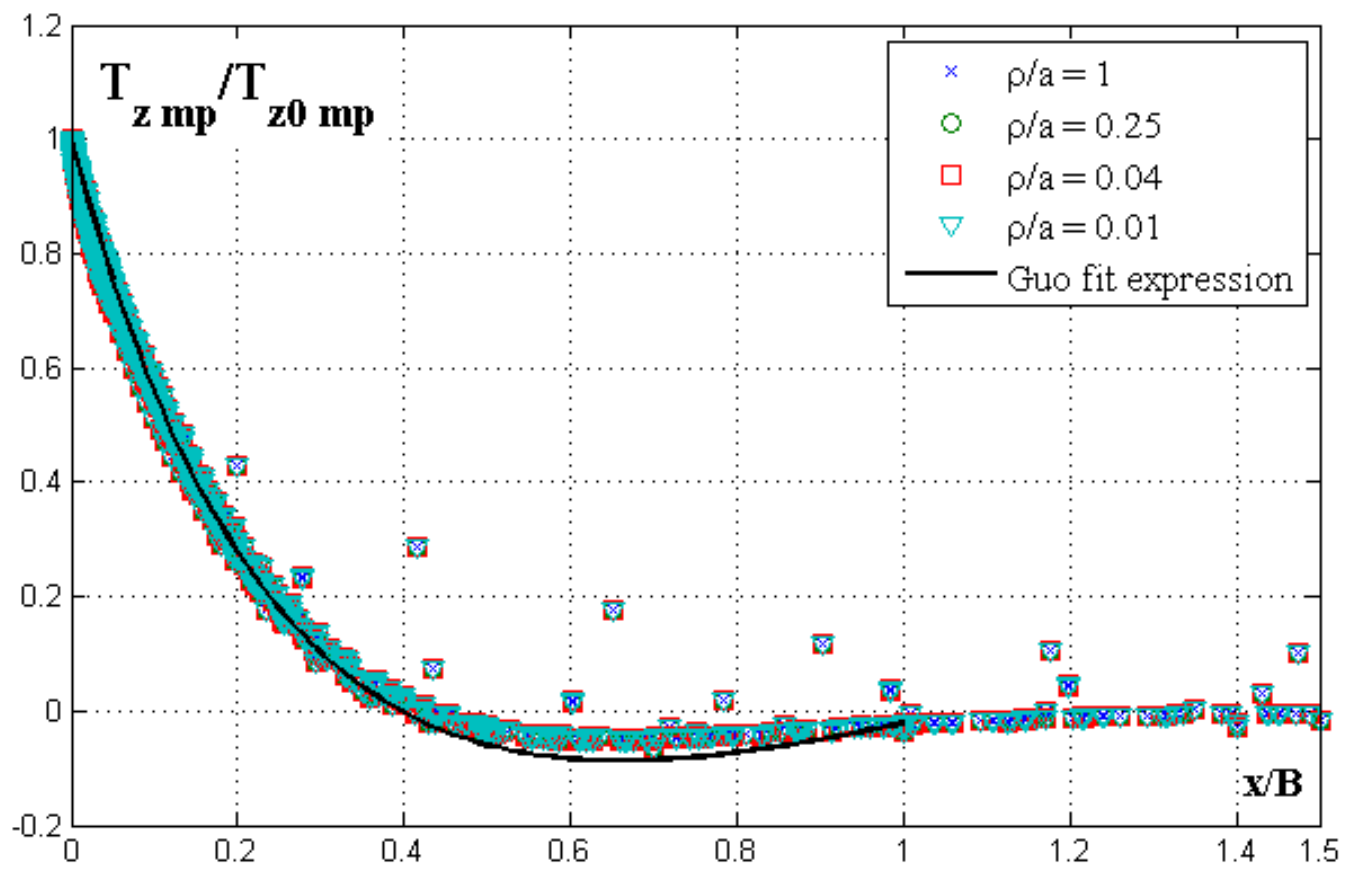

Figure 3.9 - Normalized out-of-plane constraint factor $T_{z_{\mathrm{mp}}} / T_{z 0_{\mathrm{mp}}}$ ahead of the notch tips 
along it, trying to become a through crack, then along the $x$-direction, inwards the specimen. However, although reasonable, such speculations certainly need further investigation.

\subsection{1.}

\section{D effects on notch design procedures}

It is important to verify the influence the studied 3D stress/strain fields around notch tips may have in the main failure criteria employed to design structural components, by comparing typical predictions based on them with traditional predictions made using simplified 2D hypotheses. As the only non-null stress components along notch roots are $\sigma_{y}$ and $\sigma_{z}$ (since they are free surfaces), they are both tensile principal stress components. Using the previously defined out-of plane constraint factor $T_{z}$, then

$$
\begin{aligned}
\sigma_{z} & =T_{z} \sigma_{y} \\
\sigma_{\text {Tresca }} & =\sigma_{y} \\
\sigma_{\text {Mises }} & =\sigma_{y} \sqrt{1-T_{z}+T_{z}^{2}}
\end{aligned}
$$

where

$$
\begin{aligned}
\sigma_{\text {Tresca }} & =\left(\sigma_{1}-\sigma_{3}\right) \\
\sigma_{\text {Mises }} & =\left\{\left[\left(\sigma_{1}-\sigma_{2}\right)^{2}+\left(\sigma_{2}-\sigma_{3}\right)^{2}+\left(\sigma_{3}-\sigma_{1}\right)^{2}\right] / 2\right\}^{0.5}
\end{aligned}
$$

Therefore, the transversal constraint $T_{z}$ does not affect $\sigma_{\text {Tresca }}$ but tends to decrease $\sigma_{\text {Mises }}$, as it restricts distortion despite not affecting $\tau_{\max }$ along the notch tip. Let us assume, e.g., that $\sigma_{y 0}$ at the notch root is calculated from a $2 \mathrm{D}$ solution $\left(\sigma_{0_{2 D}}=K_{t} \sigma_{n}\right)$, thus contains an intrinsic error with respect to the 3D solution. At the notch tip, the errors in Tresca and Mises stresses are:

$$
\begin{aligned}
1+\operatorname{err}_{\text {Tresca }} & =\sigma_{y 0} / K_{t} \sigma_{n} \\
1+\operatorname{err}_{\text {Mises }} & =\sigma_{\text {Mises }} / K_{t} \sigma_{n}
\end{aligned}
$$

The results presented in section 2 show that $y_{0}>K_{t} \sigma_{n}$ when the transversal constraint induced by the thickness is accounted for. Therefore, they also show that 2D stress concentration predictions are intrinsically non- 
conservative, but not that much. Figure 3.10 shows the ratio $\sigma_{\text {Mises }} / K_{t} \sigma_{n}$ varies as a function of the transversal constraint $T_{z}$ for several arbitrary errors on $\sigma_{y 0}$ calculated from 2D plane solutions. Therefore, although $\sigma_{y 0}$ predictions based on SCF $K_{t}$ calculated assuming $2 \mathrm{D}$ conditions are always non-conservative, the Mises stresses around notch tips caused by the 3D conditions that actually act there may be conservatively or non-conservatively estimated by $K_{t} \sigma_{n}$, a somewhat non-intuitive result.

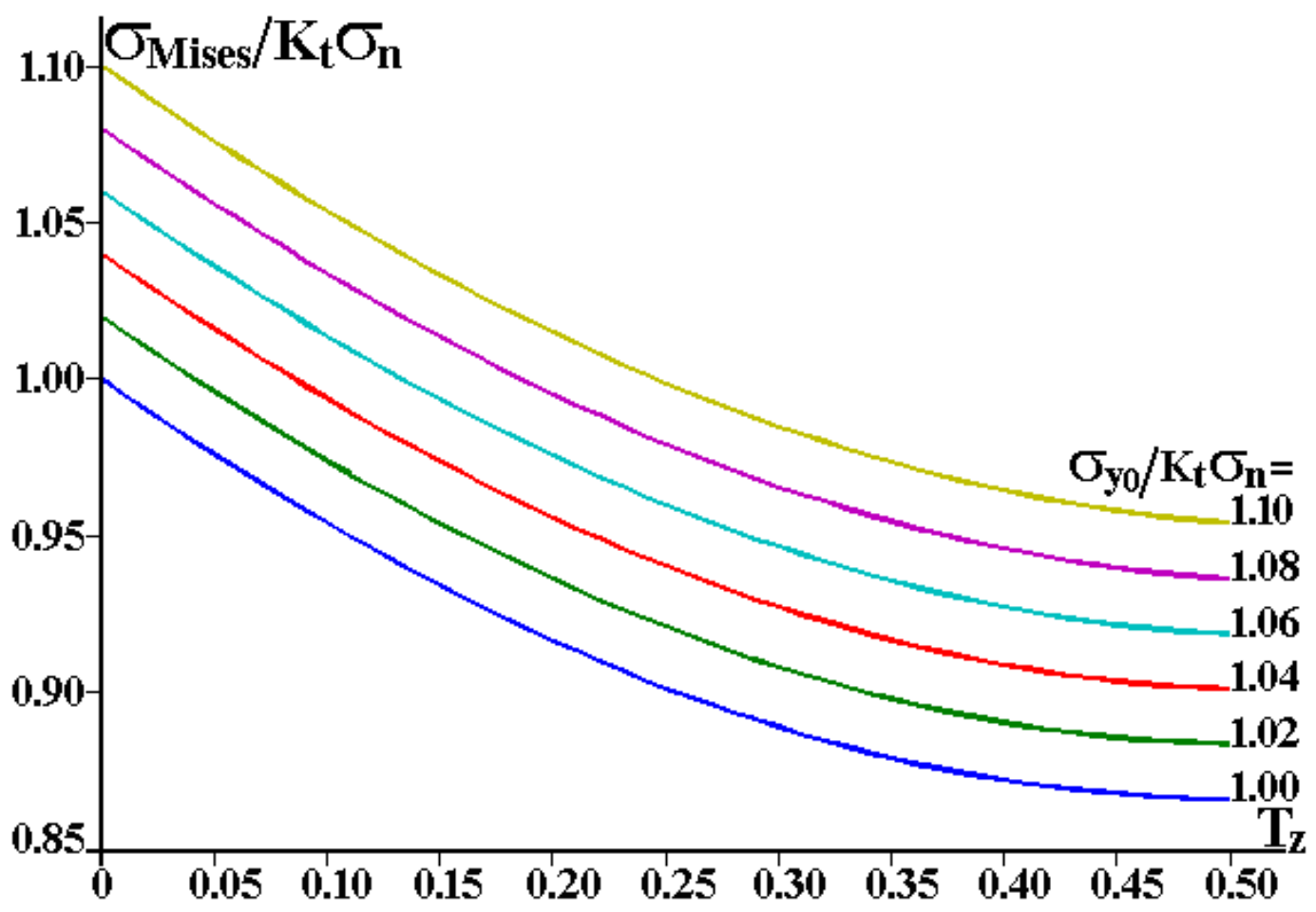

Figure $3.10-\sigma_{\text {Mises }} / K_{t} \sigma_{n}$ as a function of $T_{z 0}$, for various $\sigma_{y 0}$ values

Poisson's ratio $\nu$ is the upper bound limit to $T_{z}$, never reached for notches with finite tip radii, no matter how thick the plate is. Guo et al. proposed an estimate for the Inglis' hole $K_{\sigma_{\max }}[23]$ :

$$
K_{\sigma_{\max }} / K_{t} \approx 1+0.01 \exp \left[\frac{\nu}{0.14364+0.07 b / a}\right]
$$

Since in this case $K_{t}=1+2 a / b=1+2 \sqrt{a / \rho}$, and since this $K_{t}$ can be used as a reasonable approximation for the SCF of many other notches with size $a$ and notch tip radius $\rho$, then the maximum SCF for other notches could be estimated by:

$$
K_{\sigma_{\max }} / K_{t} \approx 1+0.01 \exp \left[\frac{\nu}{0.14364+0.14 /\left(K_{t}-1\right)}\right]
$$


However, such approximated predictions are questionable for $K_{t}>>3$. For design purposes, $1.08 K_{t}$ is a better assumption for the $K_{\sigma_{\max }}$ upper bound and, consequently, for Tresca's analyses based on 2D SCF. But as mentioned above, the maximum value of Mises around notch tips tends to be still less sensitive to such 2D approximations, see Figure 3.11 .

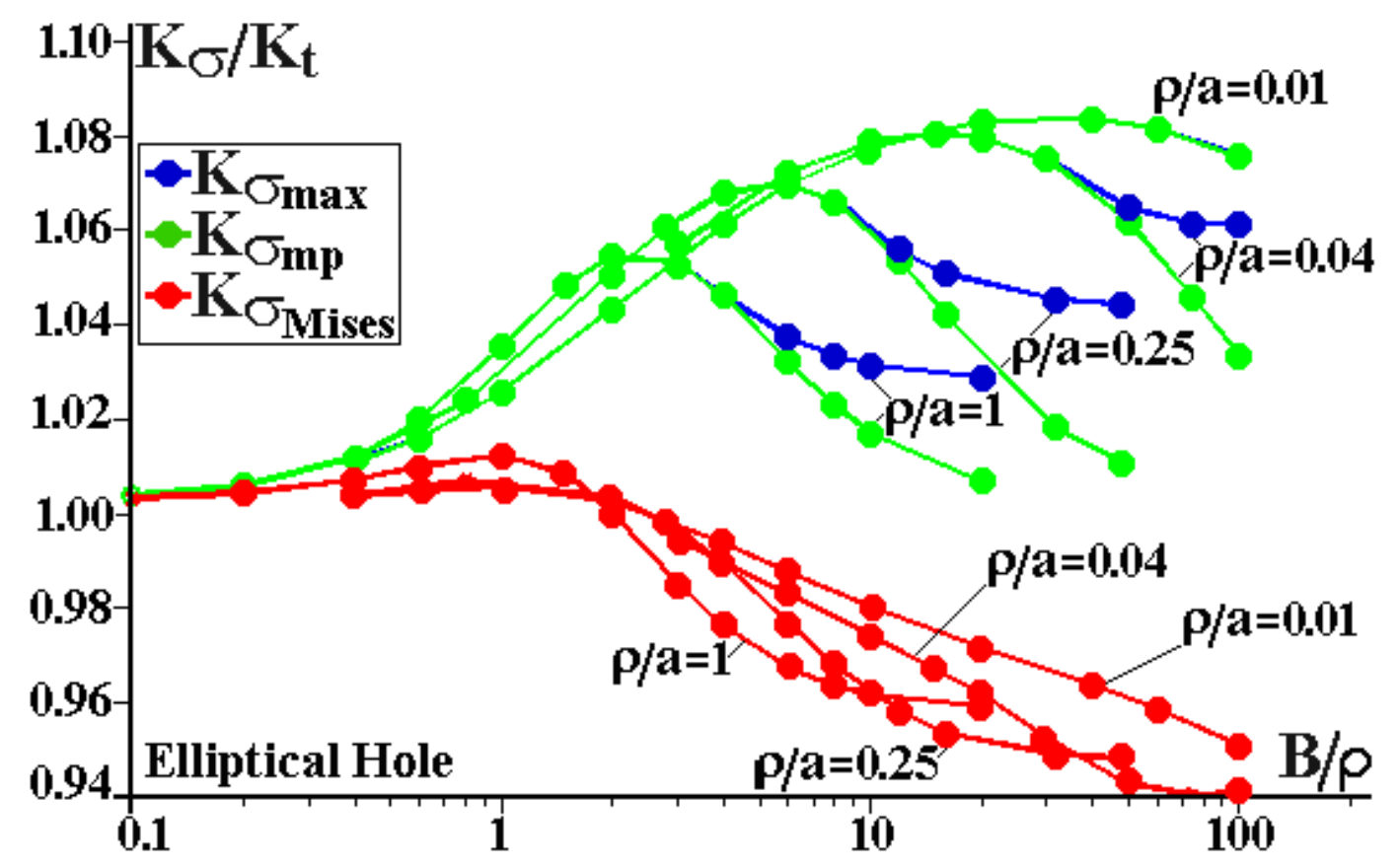

Figure 3.11 $-K_{\sigma_{\max }}, K_{\sigma_{\mathrm{mp}}}$, and $K_{\sigma_{\mathrm{Mises}}}=\sigma_{\text {Mises }} / K_{t} \sigma_{n}$ as a function of $B / \rho$

\section{2.}

\section{Crack analyses results}

As the submodel does not assume a relatively long crack (much larger than the plate thickness), it can be used to study the behavior of small cracks and to identify how different it can be from the long crack behavior. Figure 3.12 shows how much the SIF distribution along an idealized straight crack front $K_{I}(z)$ deviates from the standard 2D solution. $K_{I} / K_{I_{2 D}}$ distributions along the crack front are presented for a wide range of $B / a$ values, including short and long cracks (compared to the plate thickness). Direct comparison with previous results obtained expression for long cracks 19,22 shows that, exactly as expected, as the cracks get longer (or as their $B / a$ value decreases), the $K_{I}$ distribution along the crack front gets closer to those results (calculated assuming $K$-dominated far field boundary conditions in the Boundary layer approach).

However, relatively shallow cracks behave in a different way. Their SIF distribution tends to the $2 \mathrm{D}$ solution along most of the crack front, but 
increases close to the plate free surface. $K_{I_{\max }}$, which occurs at the middle plane for low $B / a$, dislocates towards the free surface. This behavior is better observed in Figure 3.13, and is analog to the dislocation of the maximum $K_{\sigma}$ and $K_{\epsilon}$ position in notches (see Figure 3.3).

Therefore, the difference between the short and the long crack behaviors should not be neglected when analyzing them. However, it must be noted that long cracks here mean large in comparison to the plate thickness $B$, not with the plate width $W$. The analysis of very deep cracks must include the influence of the back face plane on the LE fields ahead of the crack tip, a problem considered beyond the scope of this work.

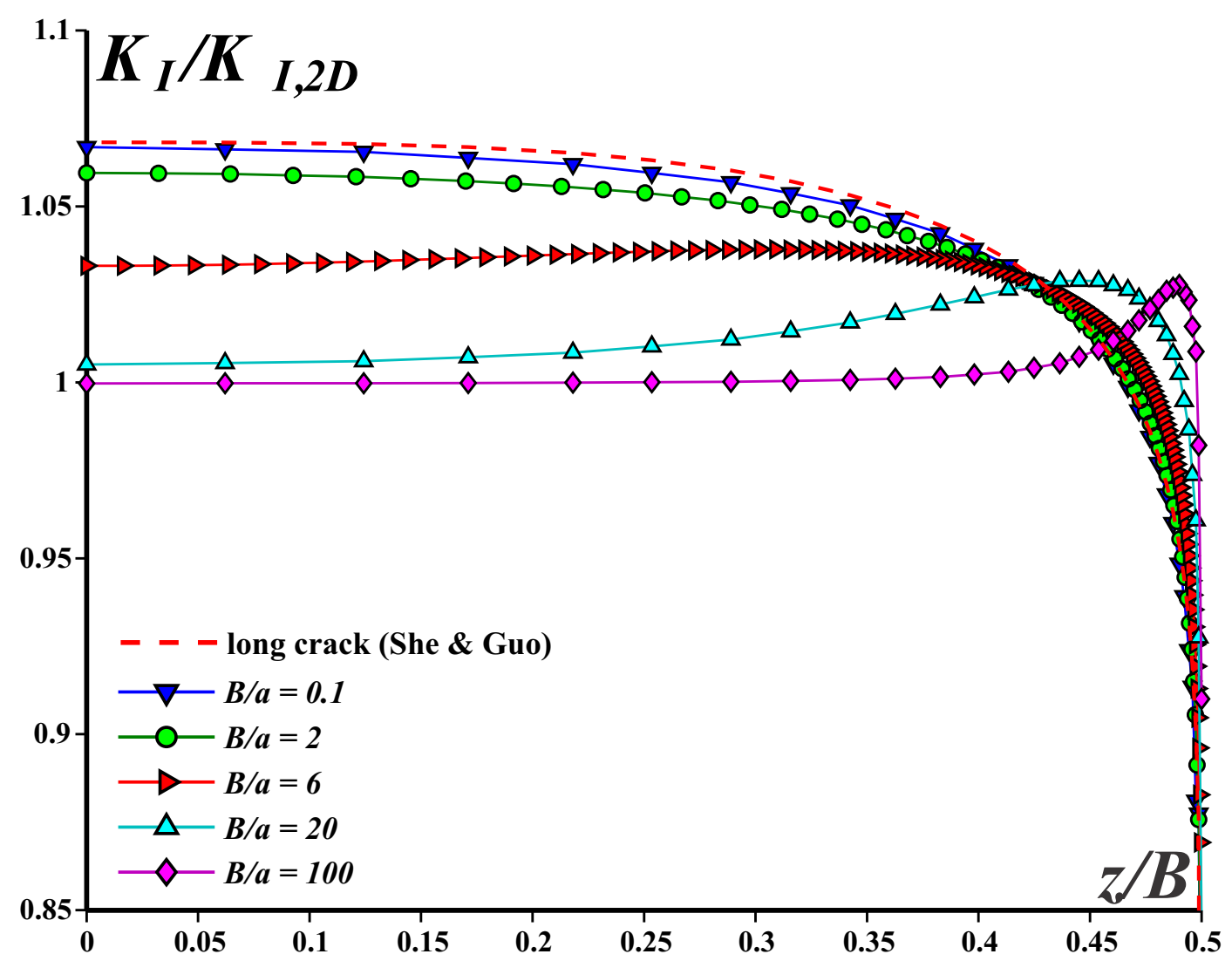

Figure $3.12-K_{I} / K_{I_{2 D}}$ distribution along the crack front for several $B / a$ ratios. The solutions are compared with She \& Guo's fit expression (eq. 1.18) for $K_{I} / K_{I_{2 D}}$ along the front of a long crack.

Figure 3.12 shows that in all cases the SIF value drops in the vicinity of the free surface. As the crack front is assumed perpendicular to the free surface in such $3 \mathrm{D}$ analyses, $K_{I}$ in fact should be null at the surface, where $z / B \rightarrow 0.5$, as previously mentioned in section 1.2 . But such a limit value could not be achieved with any reasonable level of mesh refinement. Anyway, its real importance is to force real cracks to slightly curve their fronts during their propagation, as studied later on. But before tackling this task, the $T$-stress distribution along an idealized straight crack front is presented in Figure 3.14 . 


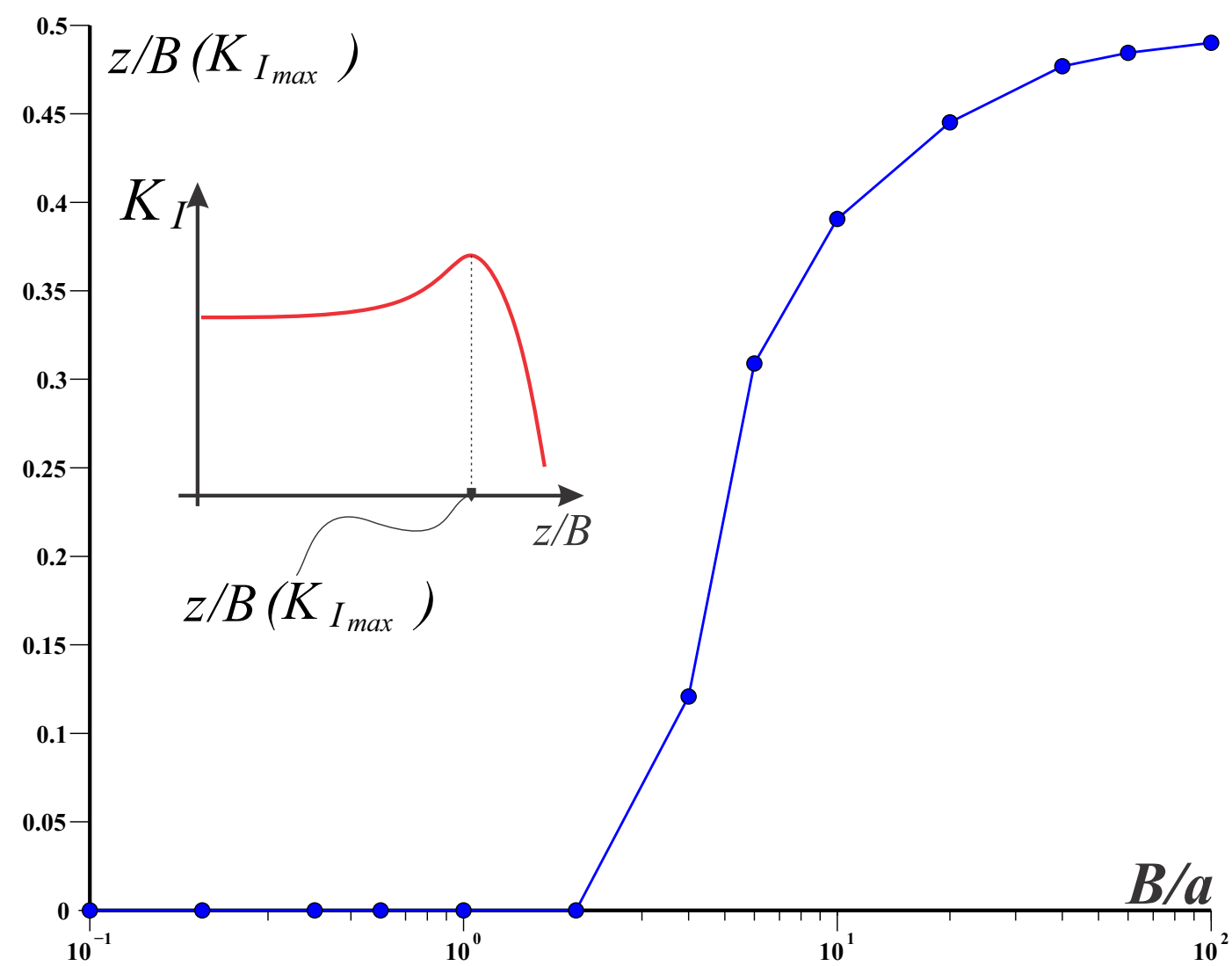

Figure 3.13 $-z / B$ coordinate along the crack front where the maximum SIF $K_{I_{\max }}$ occurs

for a wide range $0.1 \leq B / a \leq 100$ values, which include both short and long cracks. The numerical noise observed very close to the plate surface is an unavoidable consequence of the idealized straight crack front, which forces $K_{I}$ to be zero at the plate face. It should not obscure the fact that the $T$-stress distribution is almost constant along the crack face, but its value, like the $K_{I}$ value previously studied, is also dependent on the crack size. In fact, the $T$ stress shows much more variation then $K_{I}$, as $T / K_{I_{2 D}} \sqrt{\pi a}$ it goes from -0.09 to -0.15 , a $66.6 \%$ increase in terms of absolute values.

Figure 3.15 shows how the $K_{I_{\max }} / K_{I_{2 D}}$ and $K_{I_{\mathrm{mp}}} / K_{I_{2 D}}$ ratios vary with the edge crack size in this large edge-cracked plate, and compares them with its long crack $K$-dominated limit solution. This figure clearly shows how the SIF values tend to She \& Guo's long crack solution [22] for cracks with very low $B / a$, and how the SIF $K_{I_{\mathrm{mp}}}$ in the middle of the plate tends to the $2 \mathrm{D}$ solution as the cracks get shorter. Also, the separation of the $K_{I_{\max }}$ and the $K_{I_{\mathrm{mp}}}$ curves shows that even for very short cracks, with high $B / a$ ratios, $K_{I_{\max }}$ remains higher than the reference $K_{I_{2 D}}$ value (about $3 \%$ higher for the Poisson coefficient $\nu=0.3$ used in these numerical simulations).

Interesting analogies can be made if the crack is considered an elliptical notch with $\rho \rightarrow 0$. The $K_{I}$ distribution along the crack tip shown in Figure 


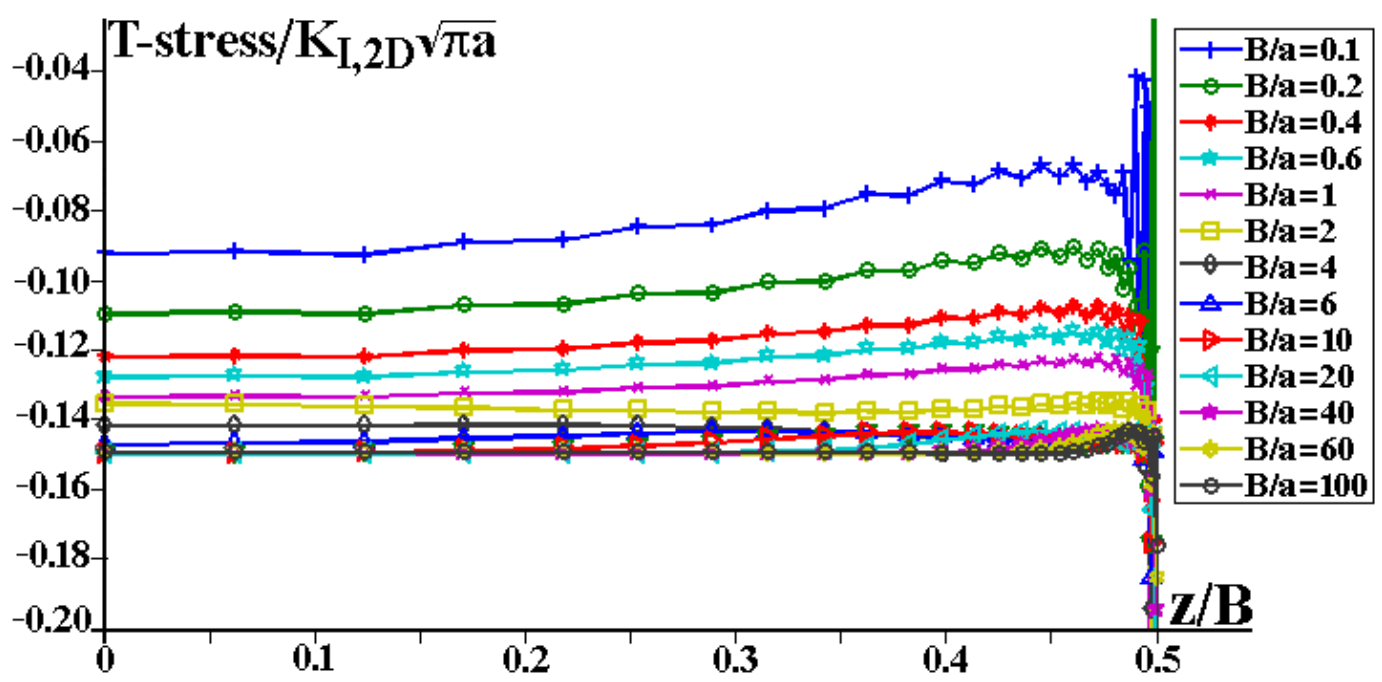

Figure 3.14 $-T$-stress $/ K_{I_{2 D} \sqrt{\pi a}}$ distribution along the front of short and long cracks

3.12 is notably similar to the $K_{\sigma}$ and $K_{\varepsilon}$ distributions along the notch root presented in Figure 3.1. They all tend to concentrate more stress in the middle of thinner plates and close to the free surface of thicker plates.

$K_{I_{\max }} / K_{I_{2 D}}$ and $K_{I_{\mathrm{mp}}} / K_{I_{2 D}}$ presented in Figure 3.15 , for instance, behave very much like $K_{\sigma_{\max }} / K_{t}$ and $K_{\sigma_{\mathrm{mp}}} / K_{t}$ after peak shown in Figure 3.4 . Even the fact that $K_{I}$ must be zero at the free surface is suggested in Figure 3.4 . where $K_{\sigma_{\text {surf }}} / K_{t}$ is shown to decrease as the notch sharpens.

Note how the submodeling technique used for analyzing this large edge cracked plate can clearly identify the size of the $K$-dominated stress field ahead of the crack tip, enhancing the role played by $\sigma_{n}$ on the $\sigma_{y}(x)$ gradient that acts there. As discussed in [24], the nominal stress $\sigma_{n}$, which is neglected on traditional LEFM analyses, may have an important effect on LE estimates for the size and shape of $p z(\theta)$, which are so important to validate LEFM predictions. Figure 3.16 illustrates how $\sigma_{y_{\mathrm{mp}}} / \sigma_{n}$, the ratio between the normal stress perpendicular to the crack faces at the middle plane of the crack front and the nominal stress varies with the relative distance $x / a$ ahead of the crack tip. At distances from the crack tip up to $x / a \approx 0.2$ the $\sigma_{y_{\mathrm{mp}}} / \sigma_{n}$ stress distribution is clearly dominated by $K_{I}$ alone, but $\sigma_{y_{\mathrm{mp}}} \rightarrow \sigma_{n}$ for distances $x / a>2$. Since this value is independent of the crack size (at least for the studied range $0.1 \leq B / a \leq 100)$, this means that estimates for the LE stress field far from the crack tip in this large edge-cracked plate should include a $\sigma_{n}$ correction for distances $x / a>0.2$. Moreover, since this analysis includes all LE stress components ahead of the crack tip, it shows that the $T$-stress term is not sufficient to account for this effect.

The graphs shown in Figures $3.17(\mathrm{a})$ to $3.17(\mathrm{c})$ illustrate how $\sigma_{y}(z) / \sigma_{n}$, 


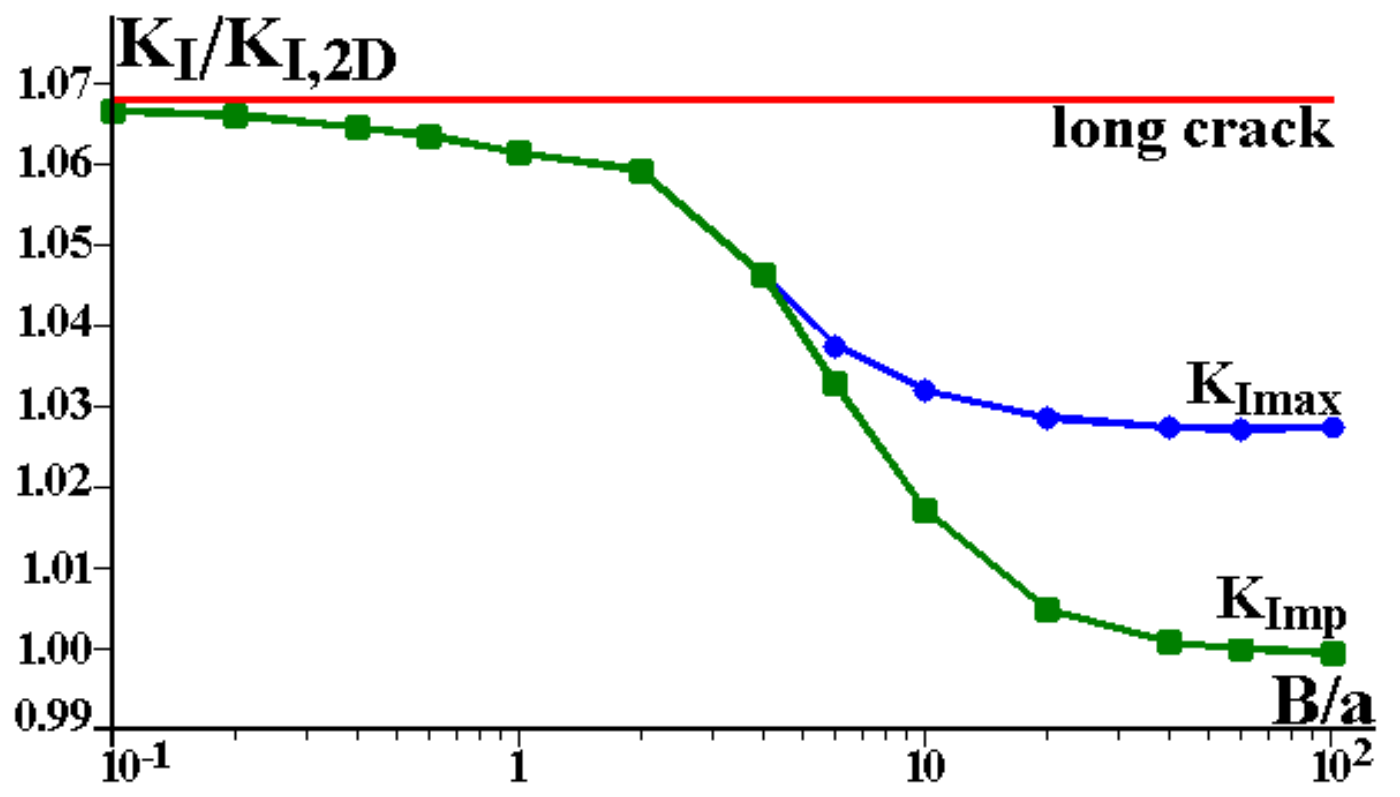

Figure 3.15 $-K_{I_{\max }} / K_{I_{2 D}}$ and $K_{I_{\mathrm{mp}}} / K_{I_{2 D}}$ variation with the crack size in large plates

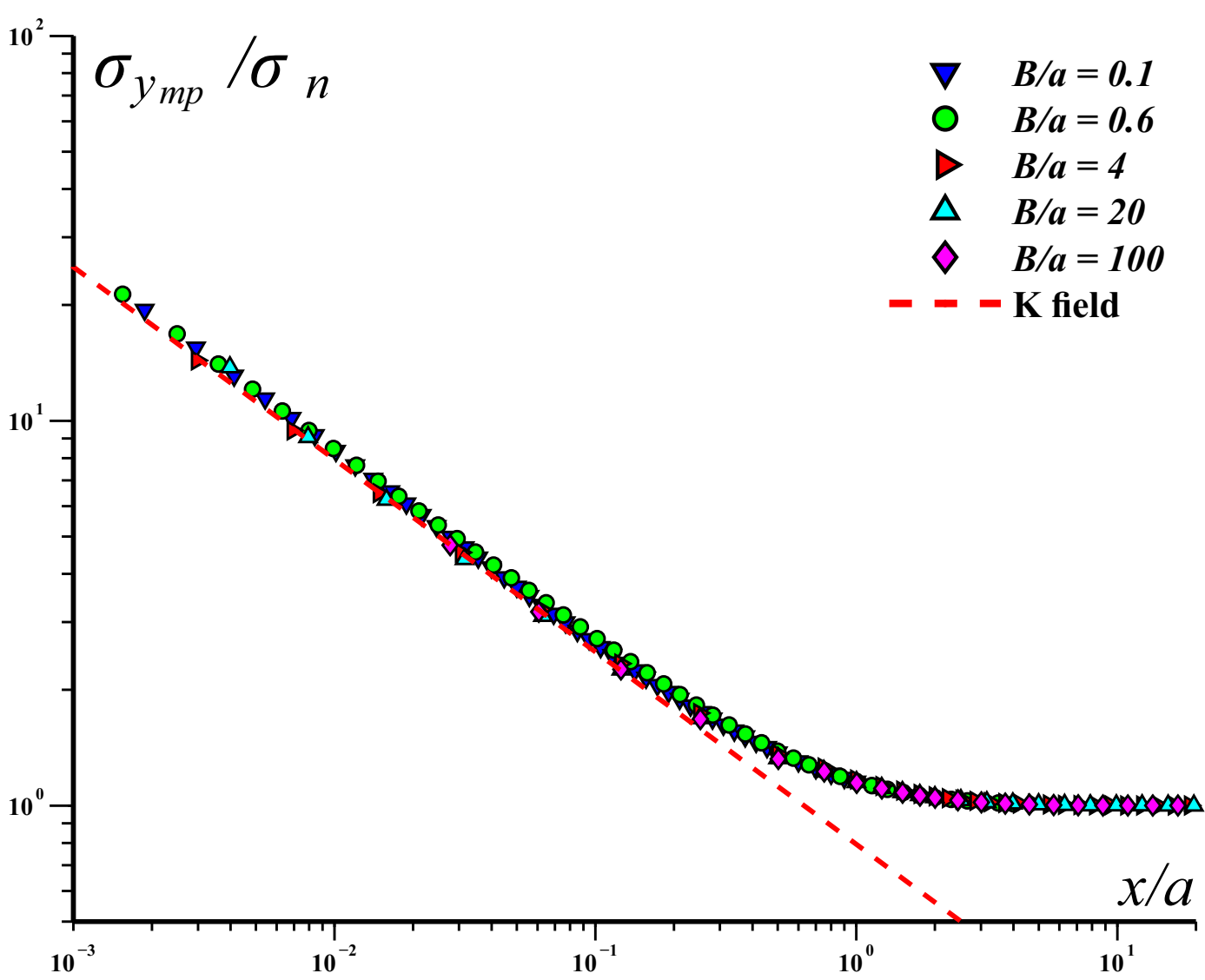

Figure 3.16 $-\sigma_{y_{\mathrm{mp}}} / \sigma_{n}$ distribution ahead of the crack tip in the large edge cracked plate 
the ratio between the normal stress perpendicular to the crack faces and the nominal stress at various positions $z / B$ along the crack front, varies with the relative distance $x / a$ ahead of the crack tip, for cracks with sizes $B / a=0.4$, 4 , and 40 . These figures show that the $z / B$ influence on the stress gradient is slightly more important close to the free surface.

Particular attention is called upon the transversal constraint behavior. In section 3.1 it was shown that the highest constraint in notched plates is achieved in their mid-plane (at $z / B=0$ ), and that $T_{z}$ increases and tends to $\nu$ as the notch becomes sharper, but never reaches this value as notch tips radii $\rho$ remain finite. Figures 3.18 and 3.19 show the behavior of the transversal constraint factor along the mid-plane of the cracked plate $T_{z_{\mathrm{mp}}} / \nu$ versus the position ahead of the crack tip, for cracks with sizes in the large range $0.1 \leq B / a \leq 100$. In Figure 3.18 , the $x$-coordinate is normalized by the plate thickness $B$; whereas in 3.19 it is normalized by the crack length $a$.

Note that at the middle point of the crack front $(x \rightarrow 0, z=0)$ the limit condition $T_{z_{\mathrm{mp}}}=\nu$ is achieved, no matter the crack size. In other words, under LE conditions, any crack (with $\rho \rightarrow 0$ ) would reach the pl- $\varepsilon$ limit value for the transversal constraint $T_{z_{\mathrm{mp}}}$ at mid-point of the crack tip. Differences can be observed, though, in the $T_{z}$ gradient ahead of the crack tip, which is a good indicative of how much material is subjected to high transversal constraints due to the crack.

For relatively long cracks, say with $B / a>6, T_{z_{\mathrm{mp}}}$ converges to a function of $(x / B)$ (better visualized in 3.18) which agrees reasonably well with eq. (1.12), fit for notches, and with Guo's long crack solution (see section 1.2). Essentially, this means that the size of the restricted zone ahead of a long crack is proportional to the plate thickness $B$. However, for shorter cracks, with higher $B / a$ ratios, the present results show that the transversal constraint level decreases more rapidly ahead of the crack tip, which can be noted in the steeper gradients of $T_{z_{\mathrm{mp}}}$ VS $x / B$ in Figure 3.18 .

The short crack limit behavior is better noted in Figure 3.19 as $B / a$ increases, $T_{z_{\mathrm{mp}}}$ tends to a function of $x / a$, meaning that the restricted zone induced by a very short crack is in fact proportional to the crack length itself. Indeed, Figure 3.19 indicates that the constrained region advances as $B / a$ grows, until it stagnates at an upper limit for $B / a \geq 20$. From this value on, the crack is no longer able to induce more transversal restriction on the specimen, and in fact the partially constrained zone is confined within a distance from the crack tip of about $x / a<10$.

However, keep in mind that under LE assumptions, the concepts here referred as long and short crack are equivalent to thin and thick plate 


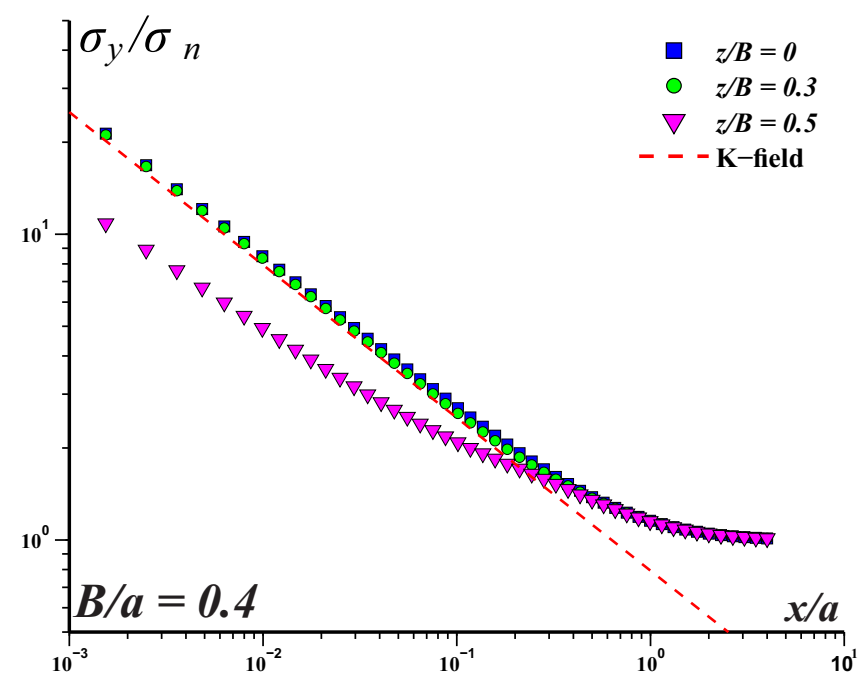

$3.17(\mathrm{a}): B / a=0.4$

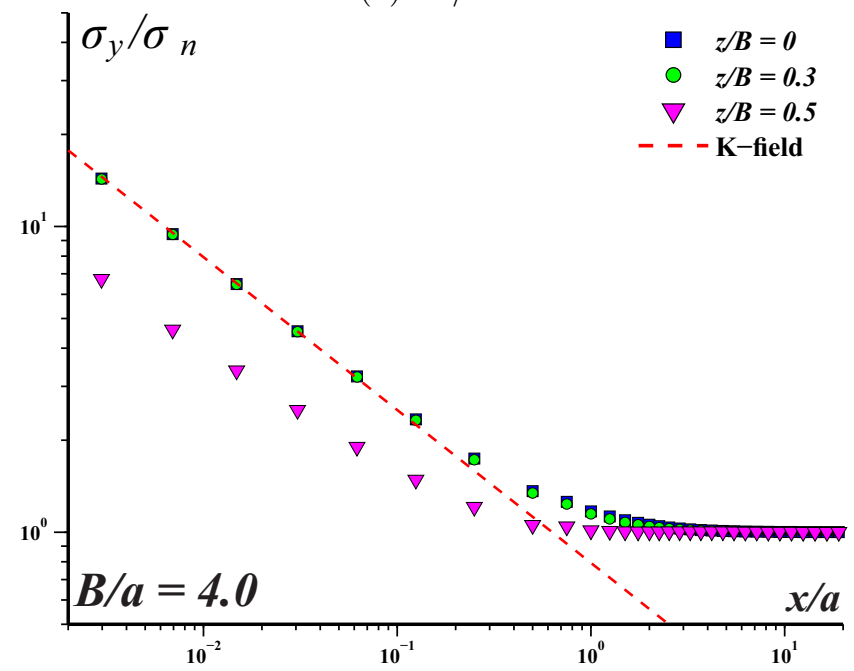

$3.17(\mathrm{~b}): B / a=4.0$

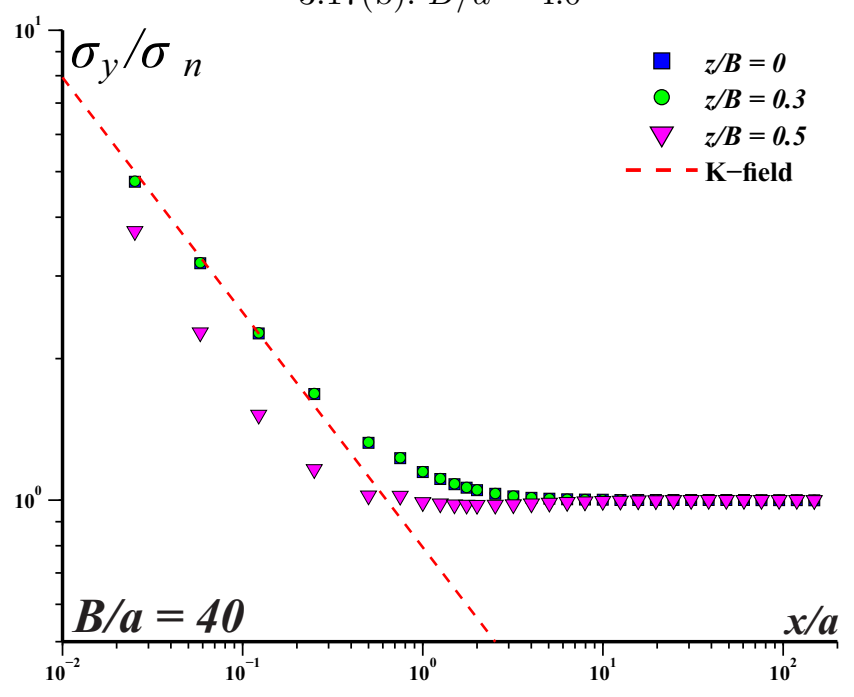

$3.17(\mathrm{c}): B / a=40$

Figure 3.17 $-\sigma_{y}(z) / \sigma_{n}$ VS $x / a$ gradient ahead of the crack tip at different $z / B$ planes, in cracked plates with different $B / a$ ratios 


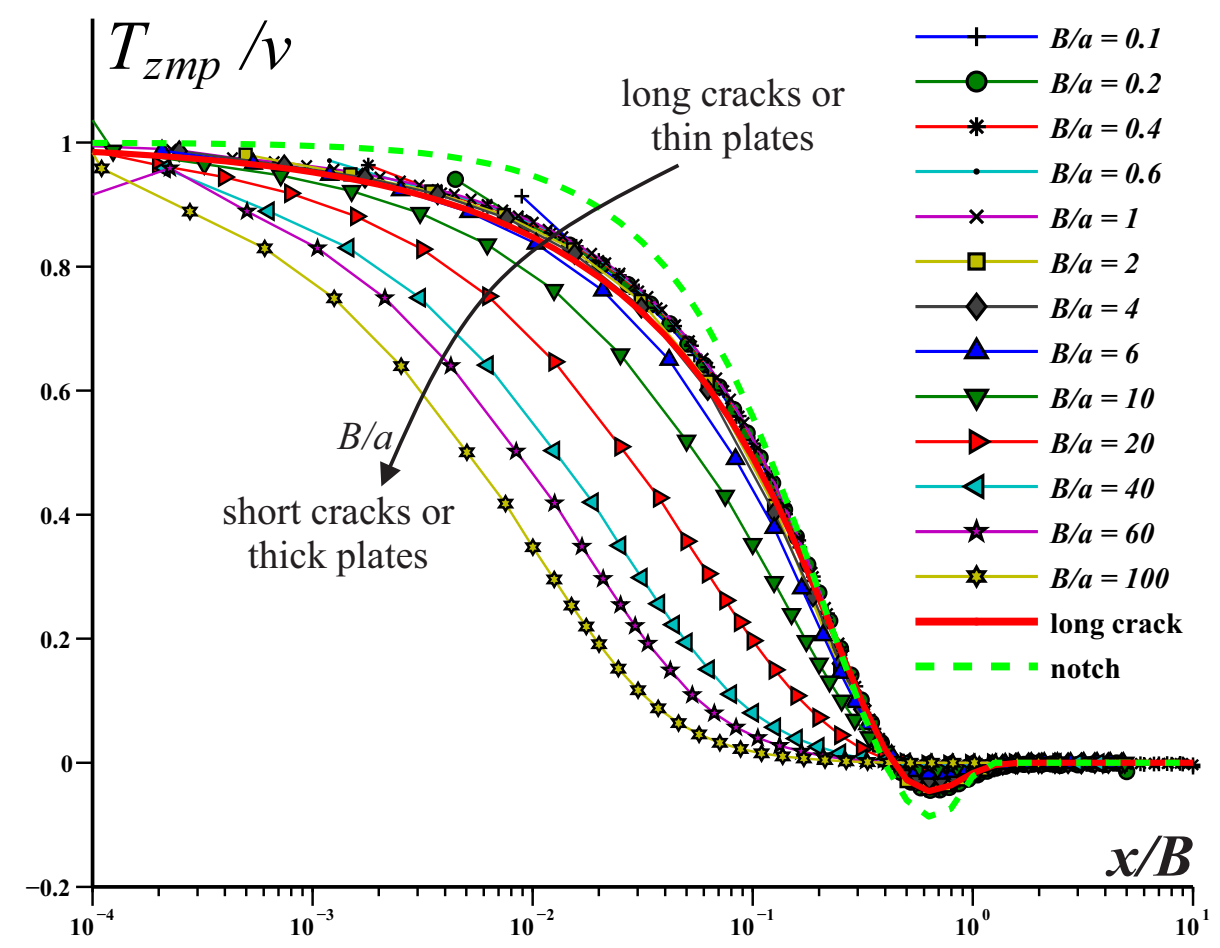

Figure 3.18 - Behavior of the transversal constraint along the mid-plane of the cracked plate $T_{z_{\mathrm{mp}}} / \nu$ as a function of the relative position ahead of the crack tip versus $x / B$

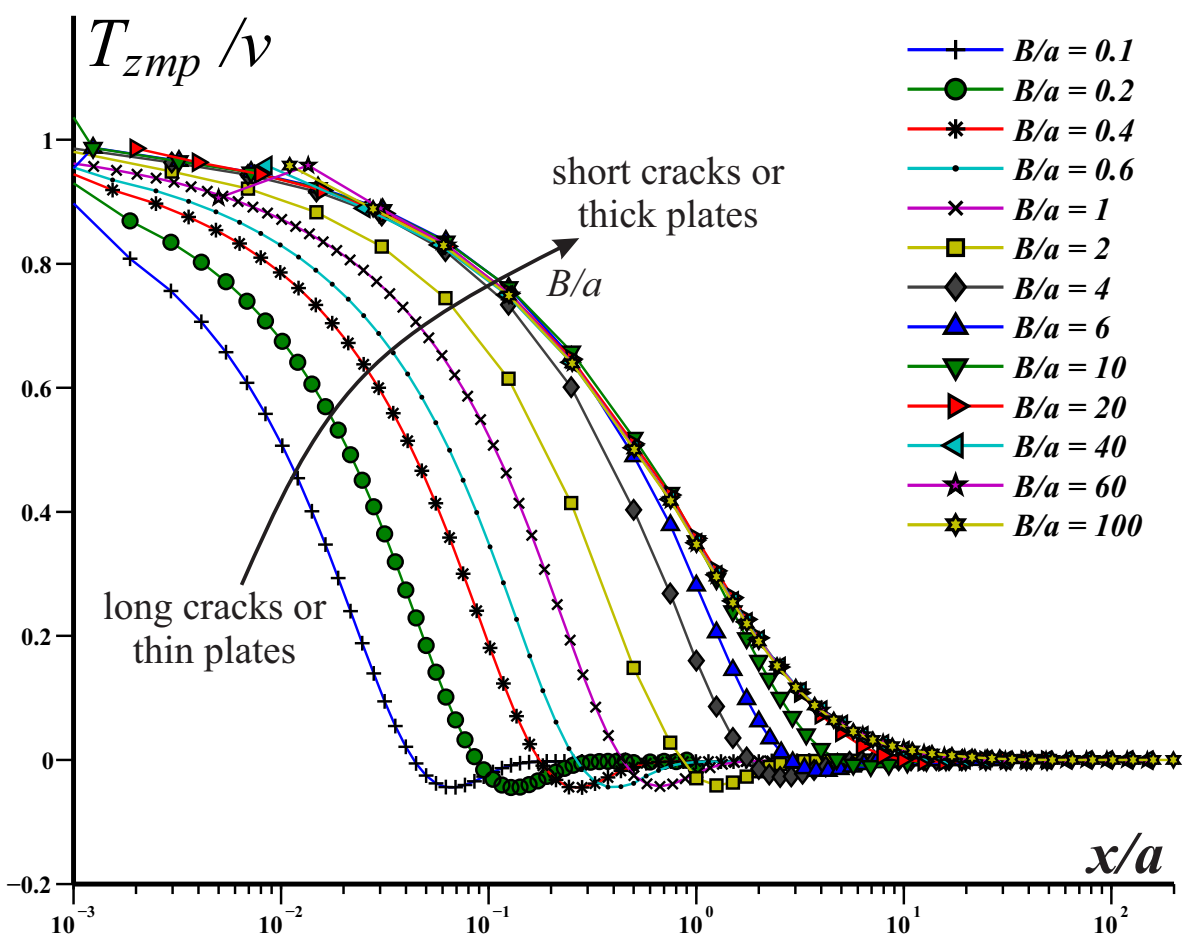

Figure 3.19 - Behavior of the transversal constraint along the mid-plane of the cracked plate $T_{z_{\mathrm{mp}}} / \nu$ as a function of the relative position ahead of the crack tip: $x / a$ 
respectively, as the only varying parameter is the $B / a$ ratio. Therefore, it is equivalent to say that in thin plates the size of the restricted zone ahead of the crack tip is proportional to the plate thickness and, in thick plates, to the crack length.

It is well known that the material toughness depends on the specimen thickness (and also on their geometry), unless they are measured under "planestrain conditions". Typical toughness tests usually yield higher values when measured in thinner specimens, which decrease as their thickness increases until reaching a material-independent "plane strain fracture toughness" value $K_{I C}$. The classical ASTM E 399 standard specifies $B>2.5\left(K_{I C} / S_{Y}\right)^{2}$ as the minimum thickness required to measure a valid $K_{I C}$. But it also specifies a relatively small crack size range $0.45<a / W<0.55$ to validate such measurements. Much less stringent, but similar requirements apply for $J$ or for critical CTOD tests. Figure 3.20 shows some data to illustrate this behavior in a Ti alloy [26].

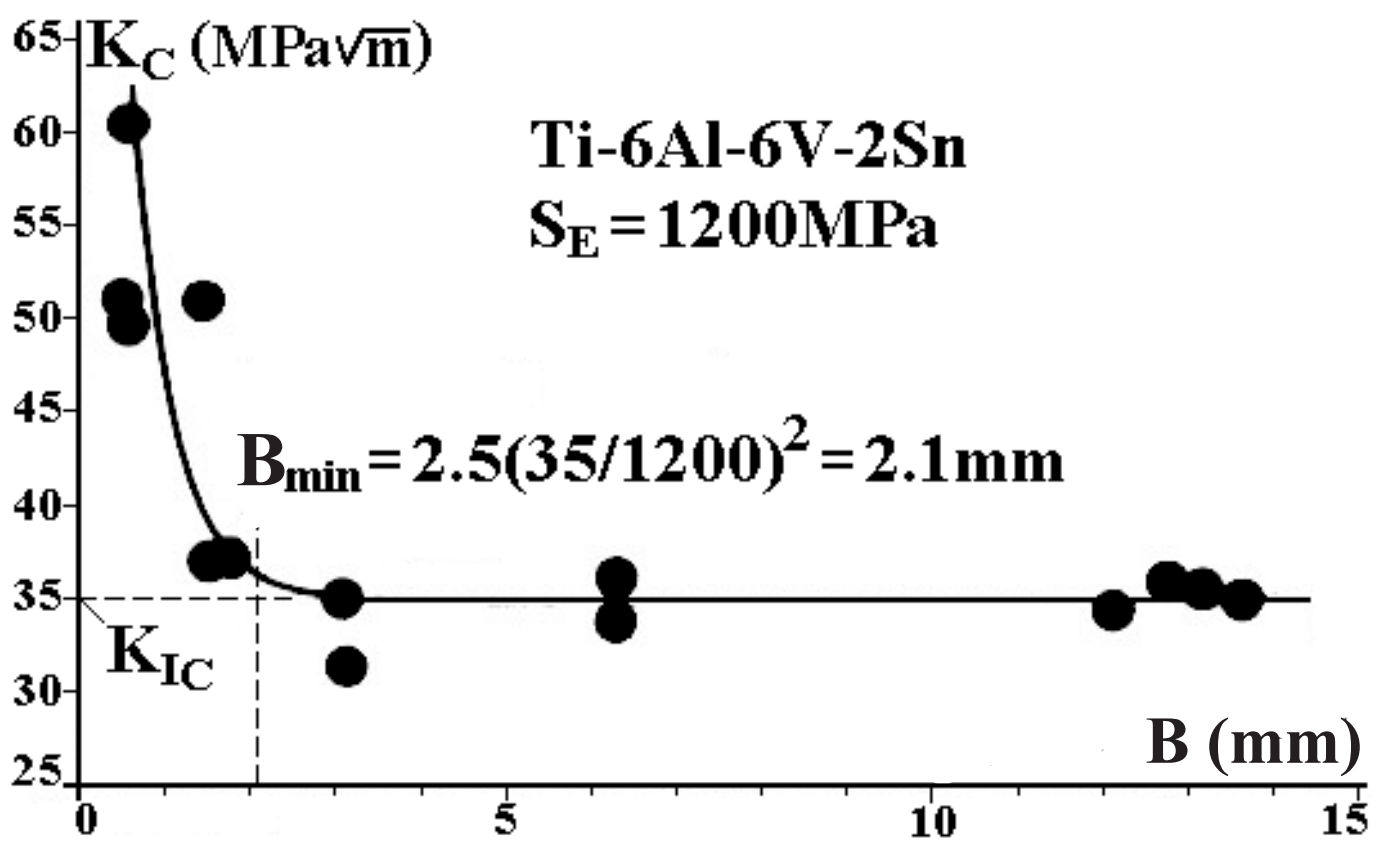

Figure 3.20 - Example of toughness $K_{c}$ dependence on the specimen thickness $B$ for a Ti-6Al-6V-2Sn alloy (see 26$]$ )

The presented results are obviously aligned with this concept. The maximal restriction, which occurs under pl- $\varepsilon$ conditions, was reached at some point by all cracks, due to the very high stress gradients ahead of their crack tip. Although the calculated values are based in LE assumptions, this indicates that the definition of dominant plane strain conditions must be associated to the amount of material that is subjected to very high transversal constraints, not only to the capacity of reaching it at some point. In fact the size of the 
highly constrained zone was found to be dependent on the plate thickness, growing with it until asymptotically reaching a stable value at some $B / a$ ratio.

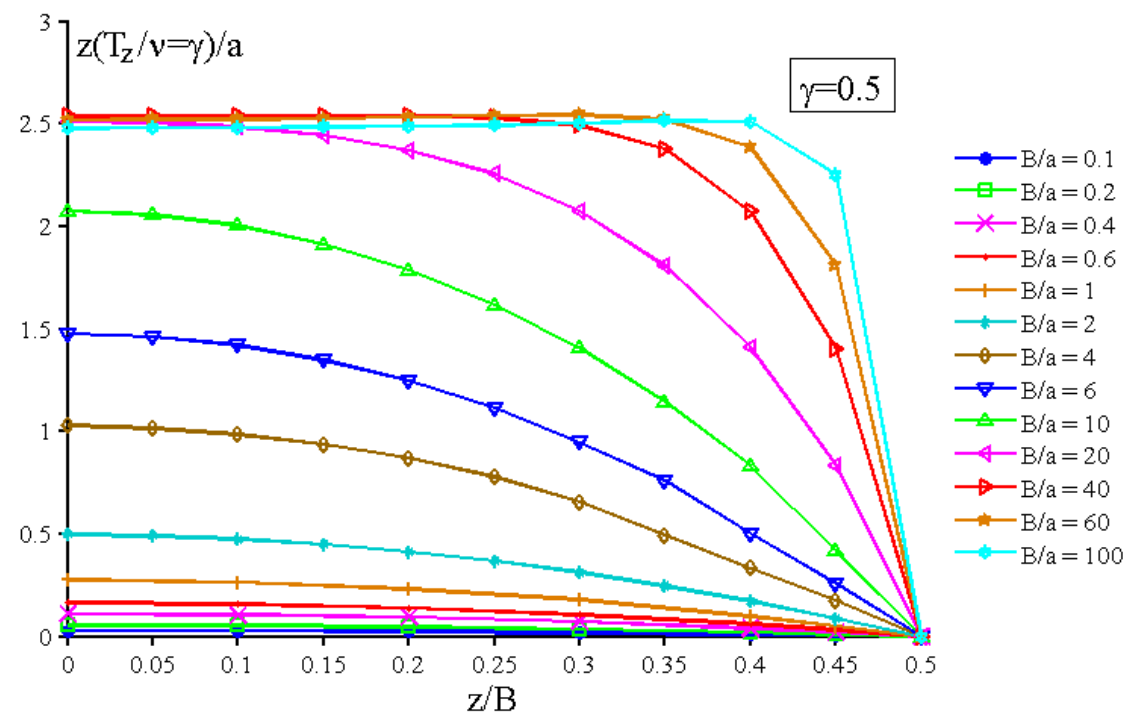

Figure 3.21 - Iso-lines where $T_{z}=0.5$ along the thickness of the plate for several cracked plates with different $B / a$ ratios

For analysis purposes, the restricted zone ahead of the crack can be considered as the area where $T_{z}>\gamma \nu$, being $\gamma$ an arbitrary value between 0 and 1 . This regon is therefore limited by the very crack front $(x=0)$ and by the line $x=x(z / B)$ where $T_{z}$ is constant and equal to $\gamma \nu$. Figure 3.21 shows the shape of such iso- $T_{z}$ lines ahead of the crack for $\gamma=0.5$ in a $x / a$ VS $z / B$ space. Note that the iso- $T_{z}$ lines are curved and penetrate deeper into the specimen material as $B / a$ increases. When $B / a$ is approximately equal to 0.4 , though, the iso- $T_{z}$ lines' depth $x / a$ grows no further, and its shape tends to a rectangle of dimensions $(c \cdot a) \times B$, with area $c \cdot a \cdot B$.

Figures 3.22 clearly shows that the area of the restricted zone normalized by $a^{2}$ tends to a straight line, with the inclination depending only on $\gamma$. Therefore, for large $B / a$ ratios, $\operatorname{Area}\left(T_{z}=\gamma \cdot \nu\right) / a^{2} \approx c \cdot a \cdot B$. Figures 3.23 and 3.24 present the same area of restriction ahead of the crack, alternatively normalized by $B^{2}$ and $B / a$.

Figure 3.24 shows the area ahead of the crack tip $x / a$ up to where $T_{z} / \nu$ falls within an arbitrary $\gamma$ value. In other words, the size of the transversally restricted zone ahead of the crack tip is very small for relatively short $B / a$ cracks, indicating that they should tend to have higher freedom to activate plasticity related phenomena, such as the toughness. Hence, short cracks tend to be less damaging not only because they are small, but also because they induce less transversal restriction ahead of their tips. As $B / a$ increases, so does the size of the restricted zone ahead of the crack tip. Thus its effect on 


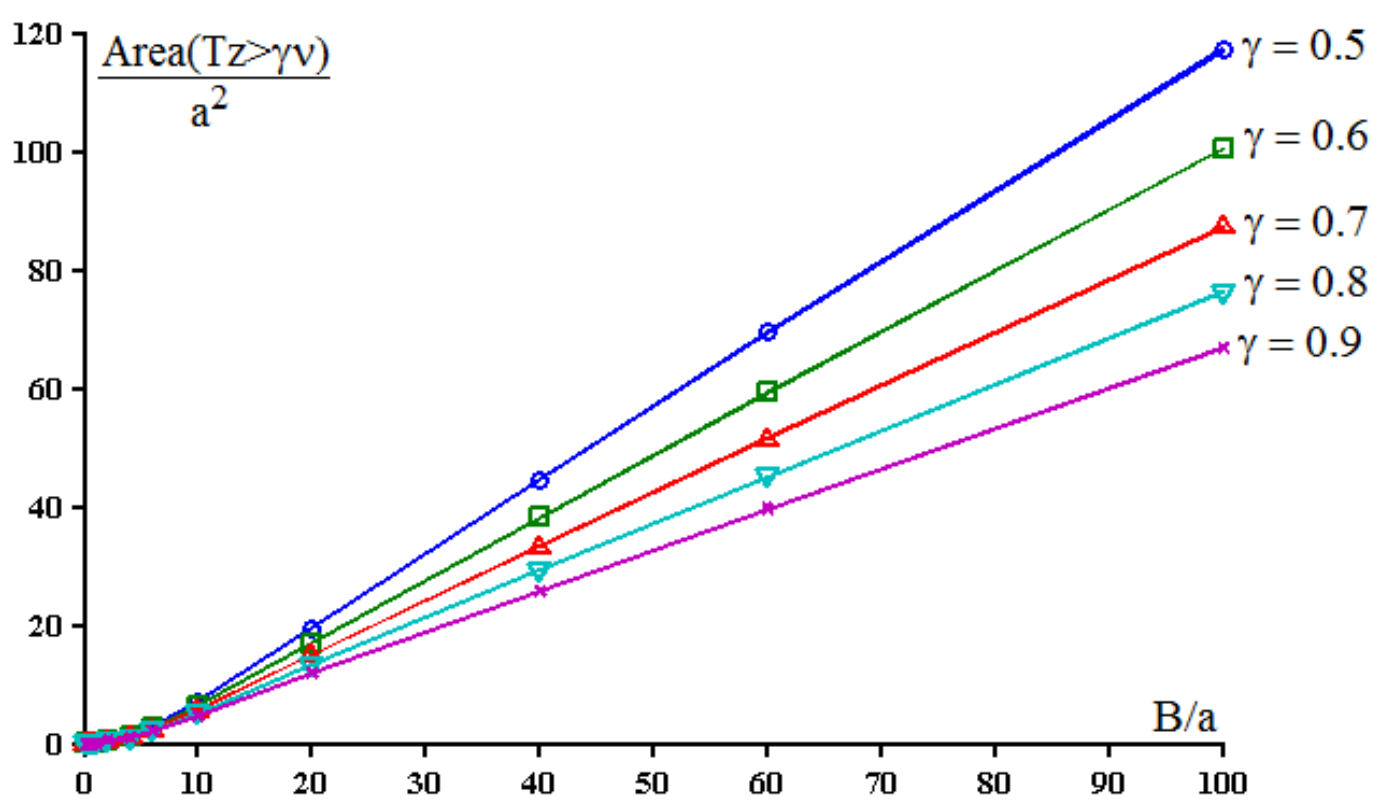

Figure 3.22 - Area ahead of the crack where $T_{z}>\gamma \cdot \nu$ normalized by $a^{2}$ versus $B / a$

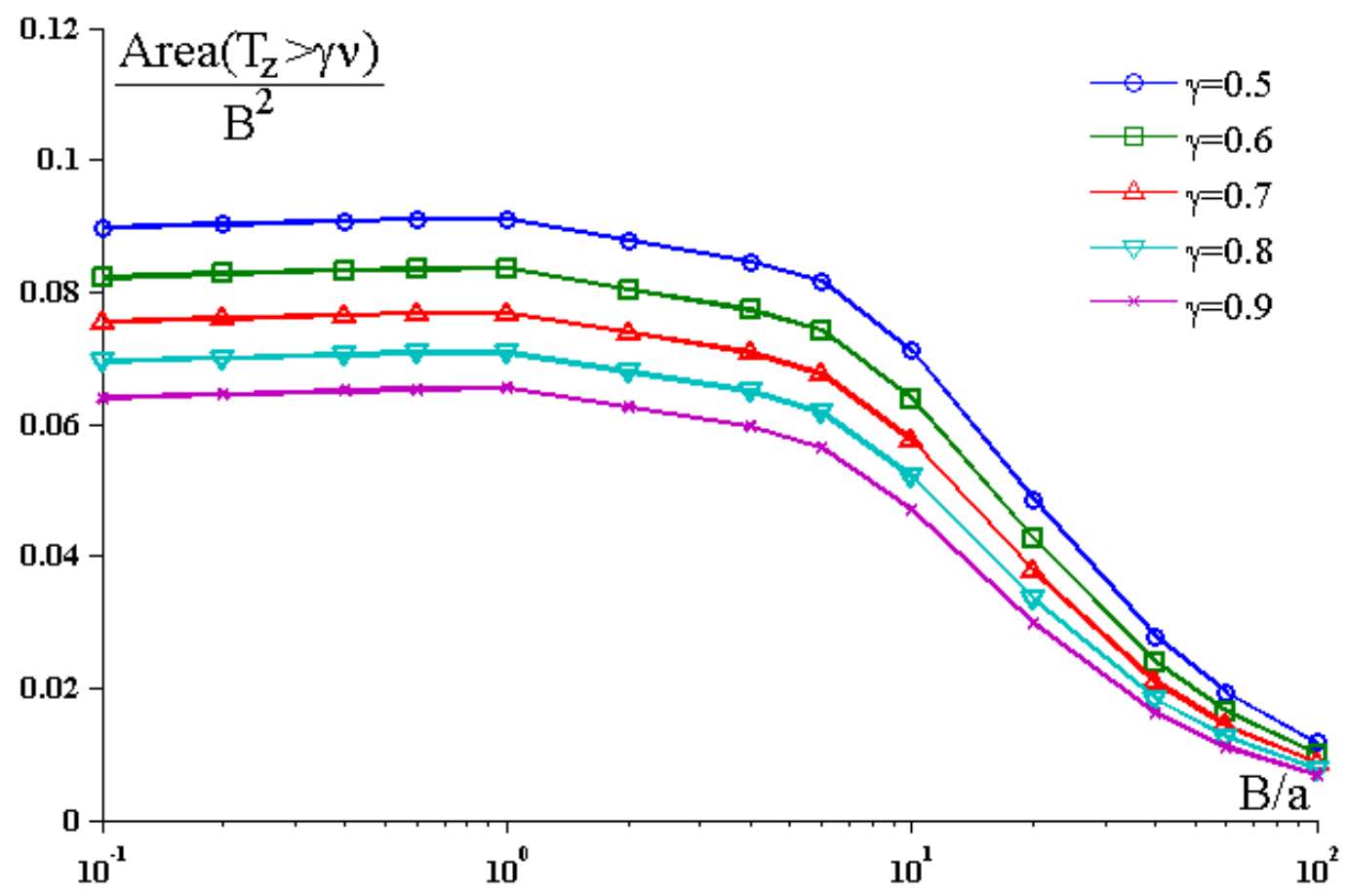

Figure 3.23 - Area ahead of the crack where $T_{z}>\gamma \cdot \nu$ normalized by $B^{2}$ versus $B / a$ 


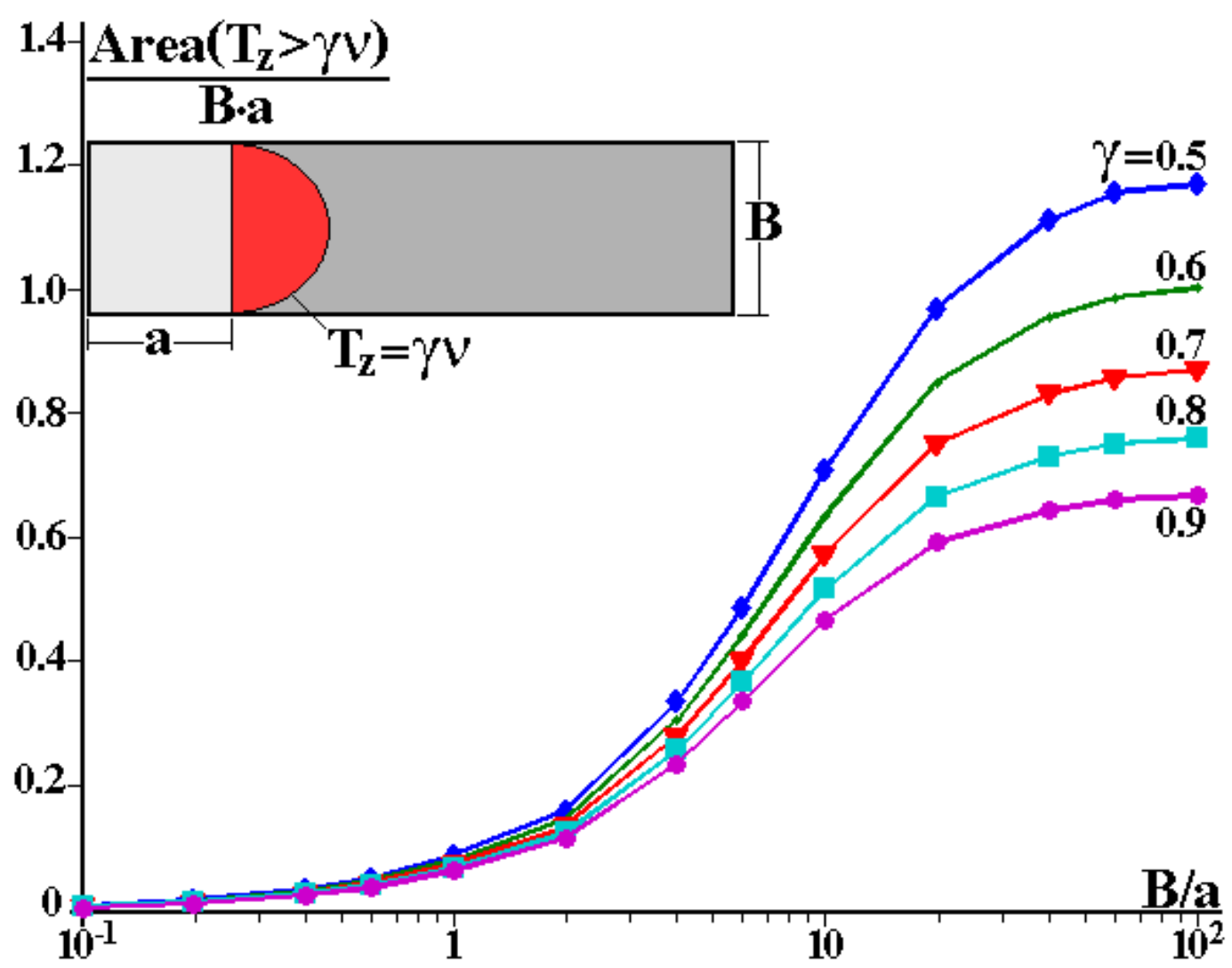

Figure 3.24 - Area ahead of the crack where $T_{z}>\gamma \cdot \nu$ normalized by $B \cdot a$ versus $B / a$

the toughness is expected to also increase, up to a critical $B / a$ value, after which the restricted zone grows no further. At this point, the toughness is expected to reach its minimal value and become independent on the crack size. Once again, although based on LE calculations, this behavior can be a reasonable explanation for why the SIF $K_{I}$ can control plasticity-induced damage mechanisms like fatigue crack propagation.

But the model studied so far is too simple to explain some features of the fatigue cracking problem, even when it is clearly $K$-controlled. Indeed, even if the crack somehow originates with a perfectly straight front on the plate edge, experimental observations show that the propagating crack front is in fact curved. The next section explores this problem, and studies how an initially ideally straight crack front must curve as the crack grows by fatigue.

\section{3.}

\section{Crack front curvature development}

Figure 3.25 shows how the SIF ratio $K_{I}(z) / K_{I_{2 D}}$ varies along the crack front with increasing values of $a_{\text {surf }}$ for the particular case of $a_{0} / B=0.02$ and of a Paris' exponent $n=2$, and also the crack front shape evolution (see 
Figure 2.16(b) along the plate thickness for the very same crack incremental stages, quantified by the ratio $\left(a(z)-a_{\min }\right) / B$. The configurations assumed by the crack front while it propagates from the initially straight shape with $a_{0} / B=0.02$ show first an anti-tunneling and then a tunneling effect, driven by the non-uniform $K_{I}(z)$ distribution along the crack front at each crack increment. This non-intuitive behavior occurs because the crack front naturally curves itself looking for a more uniform SIF distribution along it. The nonuniform SIF distribution along the initially straight crack front tends to disappear after the crack propagates for a while and gradually assumes its characteristic slight curved front. Figure 3.26 shows similar results for a higher Paris exponent, $n=4$.

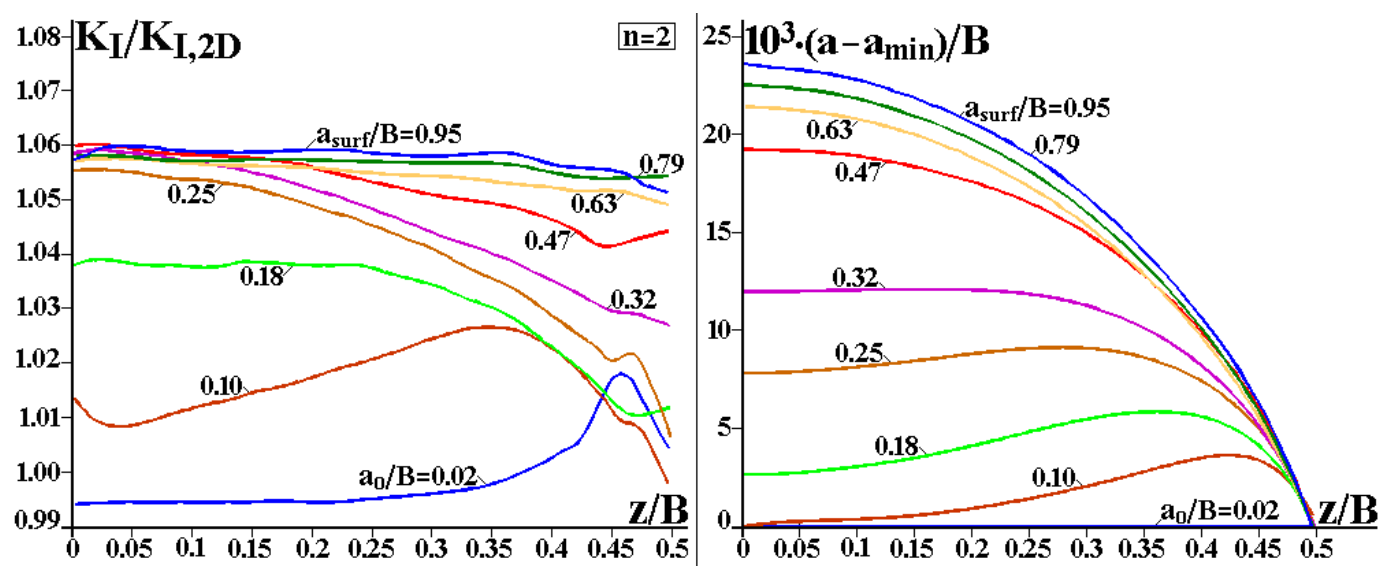

Figure 3.25 - Crack front shape evolution for $a_{0} / B=0.02$ and $n=2$

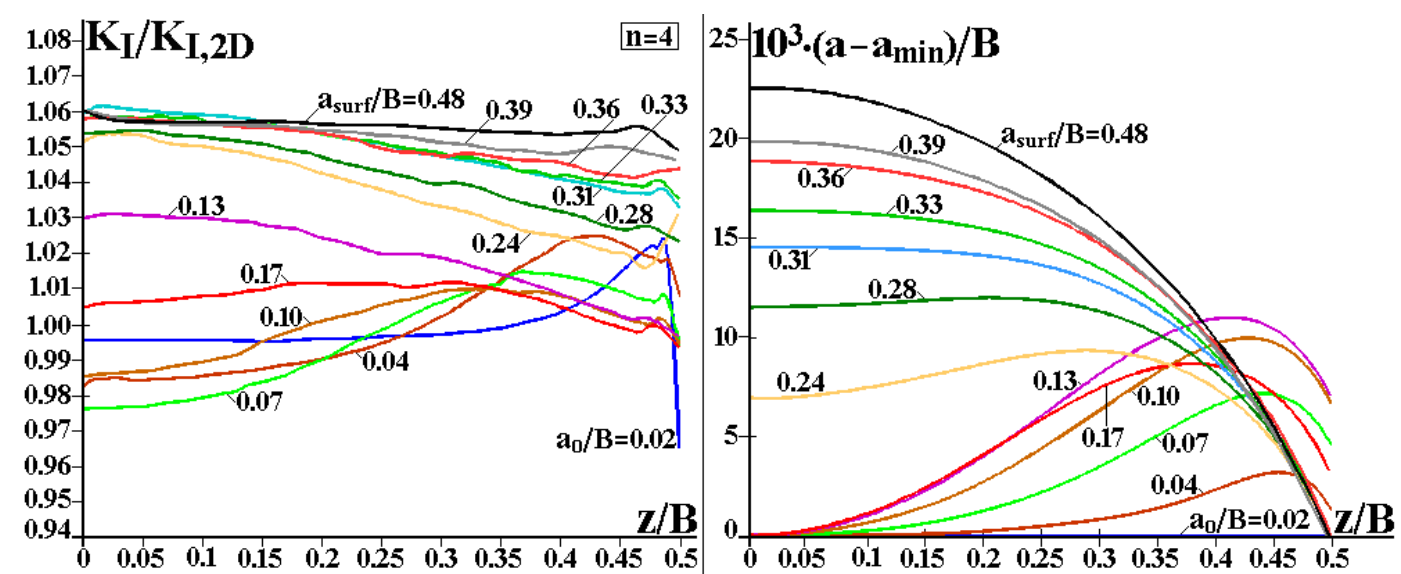

Figure 3.26 - Crack front shape evolution for $a_{0} / B=0.02$ and $n=4$

It should be noted that both for $n=2$ and for $n=4$ the cracks start propagating close to the plate free surfaces, exactly where their SIF values along the crack front for high $B / a$ ratios in Fig. 3.12 are maxima. Hence, in this particular $a_{0} / B=0.02$ case the crack first experiences the anti-tunneling behavior schematized in Fig. 2.16(b), in a more pronounced way for $n=4$ 
than for $n=2$. Moreover, as the crack front advances and curves, the SIF distribution along the crack front $K_{I}(z)$ grows flatter, until each reaches a steady state value approximately $6 \%$ higher than the $2 \mathrm{D}$ solution for both studied Paris' exponent $n$.

Figure 3.27 shows the stable $\left[a(z)-a_{\text {min }}\right] / B$ ratio along the curved crack front for all studied cracks, achieved after the transient from their initially straight fronts. Let us assume, for instance, that the difference between the maximum and the minimum crack size $a_{\max }-a_{\min }$ can be taken as a descriptive parameter of the (curved) steady state crack front, and that $\Delta a_{\text {trans }}$ is the distance the crack covers before achieveing a steady front shape (i.e. the transient propagation distance).

Figure 3.28 shows that all studied cracks achieve a steady state $\left[a_{\max }-\right.$ $\left.a_{\min }\right] / B$ ratio that is more or less independent of $a_{0}$ and $n$, after having grown for a distance of about $0.6 \cdot\left[a_{\text {surf }}-a_{0}\right] / B$. However, note that cracks that initiate at a deeper $a_{0} / B$ achieve a steady state front shape sooner than the shallower cracks, presenting smaller $\Delta a_{\text {trans }}$. Cracks that propagate under $n=4$ achieve such stable regime shape sooner than cracks that propagate under $n=2$, as well.

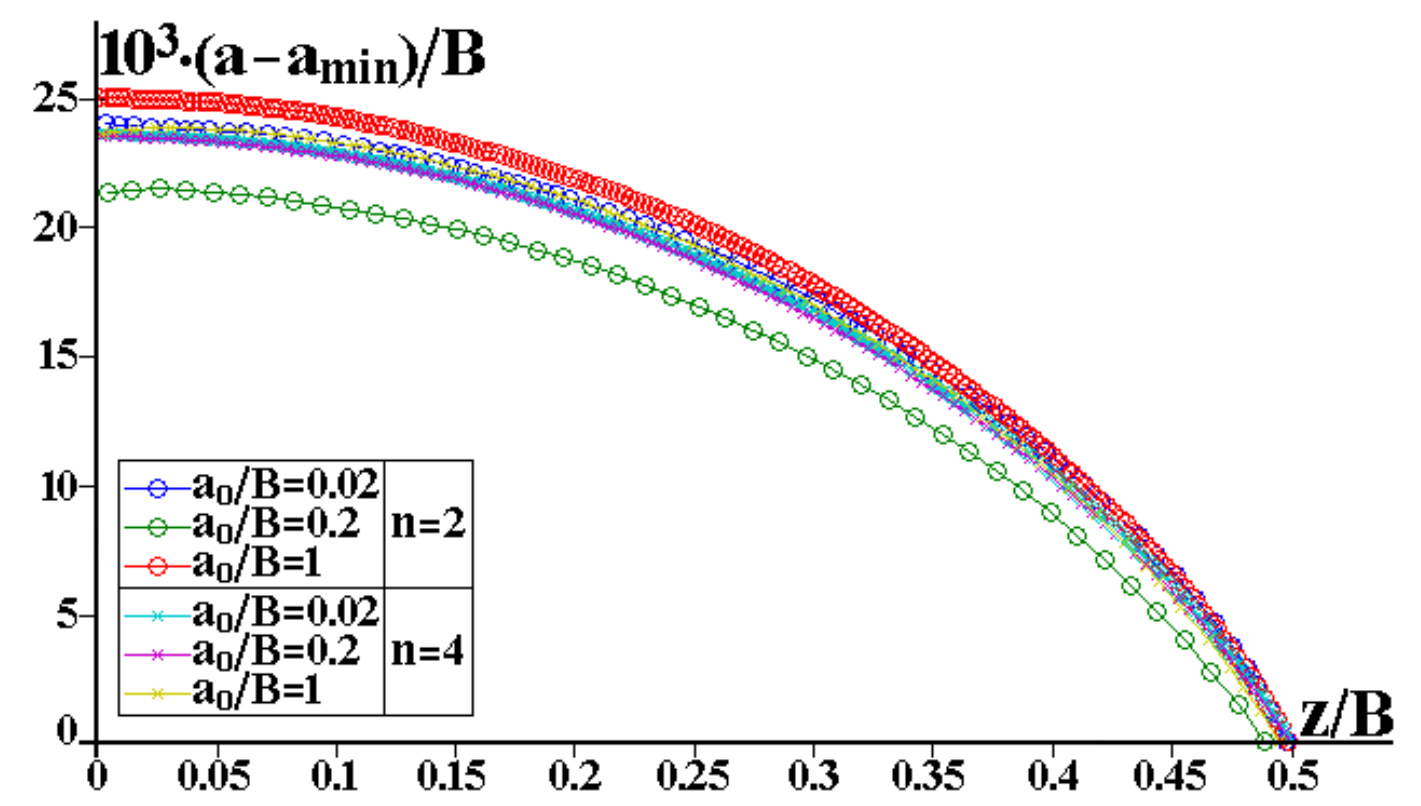

Figure 3.27 - Slightly curved front shapes under steady growth conditions

The deeper cracks with $a_{0} / B=0.2$ and 1.0 presented a more pronounced advance in the plate interior from the beginning of their propagation from an initially straight front, while the shallower ones with $a_{0} / B=0.02$ presented a higher advance close to their borders. This behavior is consistent, since the shallower cracks tend to have $K_{I}$ peaks close to their free surfaces, while the deeper ones have maximum $K_{I}$ values in the middle of their fronts. 


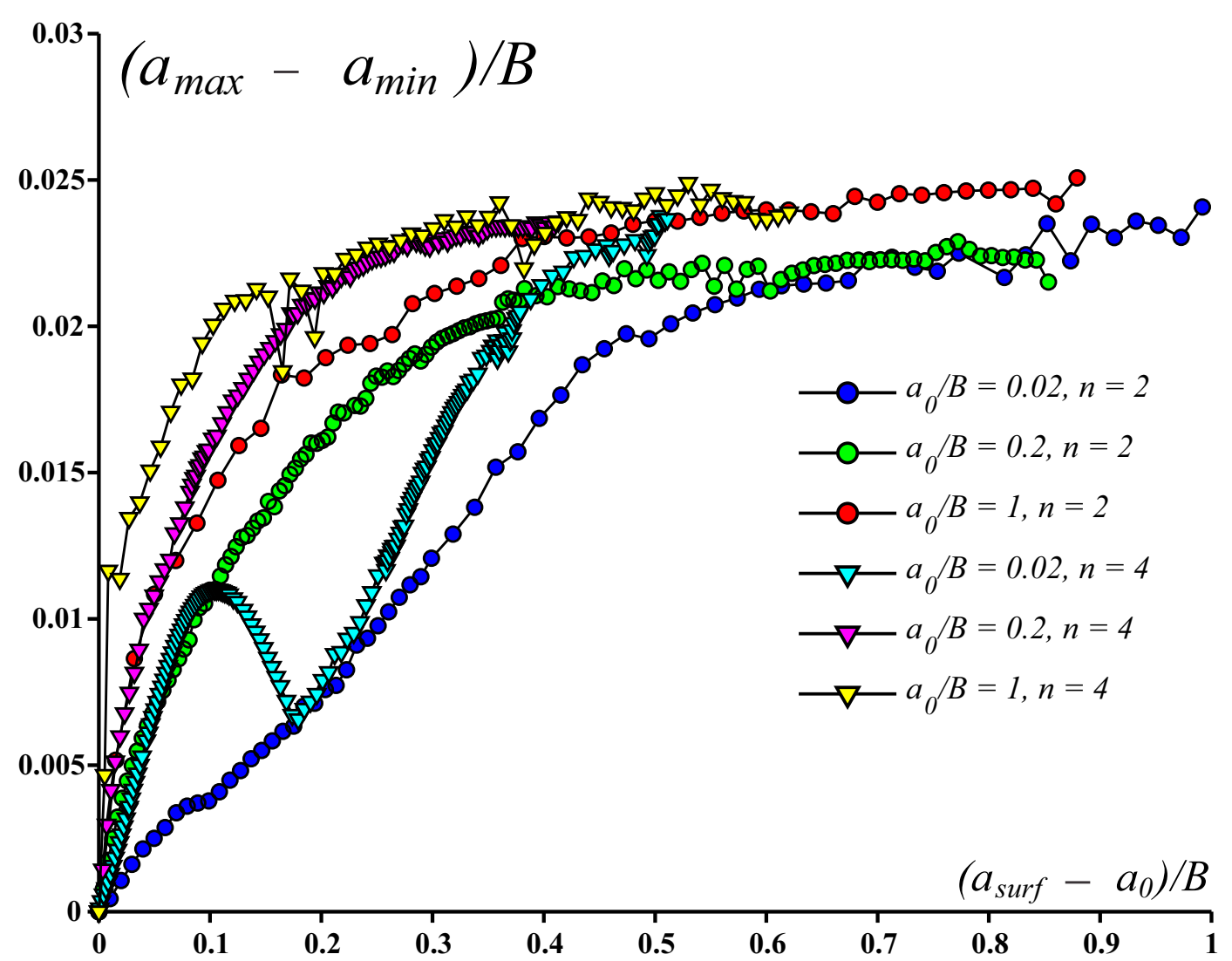

Figure $3.28-\left(a_{\max }-a_{\min }\right) / B$ variation as the crack grows from an initially straight front $a_{0}$.

Figure 3.29 shows how the ratio $K_{I_{\max }} / K_{I_{2 D}}$ varies as the cracks propagate from their initially straight fronts with size $a_{0}$. It is worth mentioning that, except for the shortest studied crack with size $a_{0} / B=0.02$, the cracks are well represented by the straight front crack solution. The differences between this particular case and the others are caused by the intense shape changes suffered by the crack front along the early propagation stages, easily observed in figures 3.25 and 3.26 . The presented results show that the transient crack propagation distance $\Delta a_{\text {trans }}$ is closely related to $a_{0} / B$ and fairly independent of $n$. Table 3.1 shows the approximate values obtained for $\Delta a_{\text {trans }}$.

Table 3.1 - Transient distance $\Delta a_{\text {trans }} / B$ covered by the crack before achievement of steady front shape for different initial lengths $a_{0} / B$

\begin{tabular}{|l|lll|}
\hline$a_{0} / B$ & 0.02 & 0.2 & 1 \\
\hline$\Delta a_{\text {trans }} / B$ & 0.25 & 0.1 & 0 \\
\hline
\end{tabular}

Figure 3.30 also shows that results obtained for long cracks with straight fronts in 18, 19,22, are reasonably good approximations for $K_{I_{\max }}$. Finally, Fig. 3.30 shows the angle $\beta$ between the crack front and the free surface. In the present work, it was found that after a short propagation distance, $\beta$ stabilized in a fixed value (around $98^{\circ}$ ), for all simulated cases. The obtained results are 


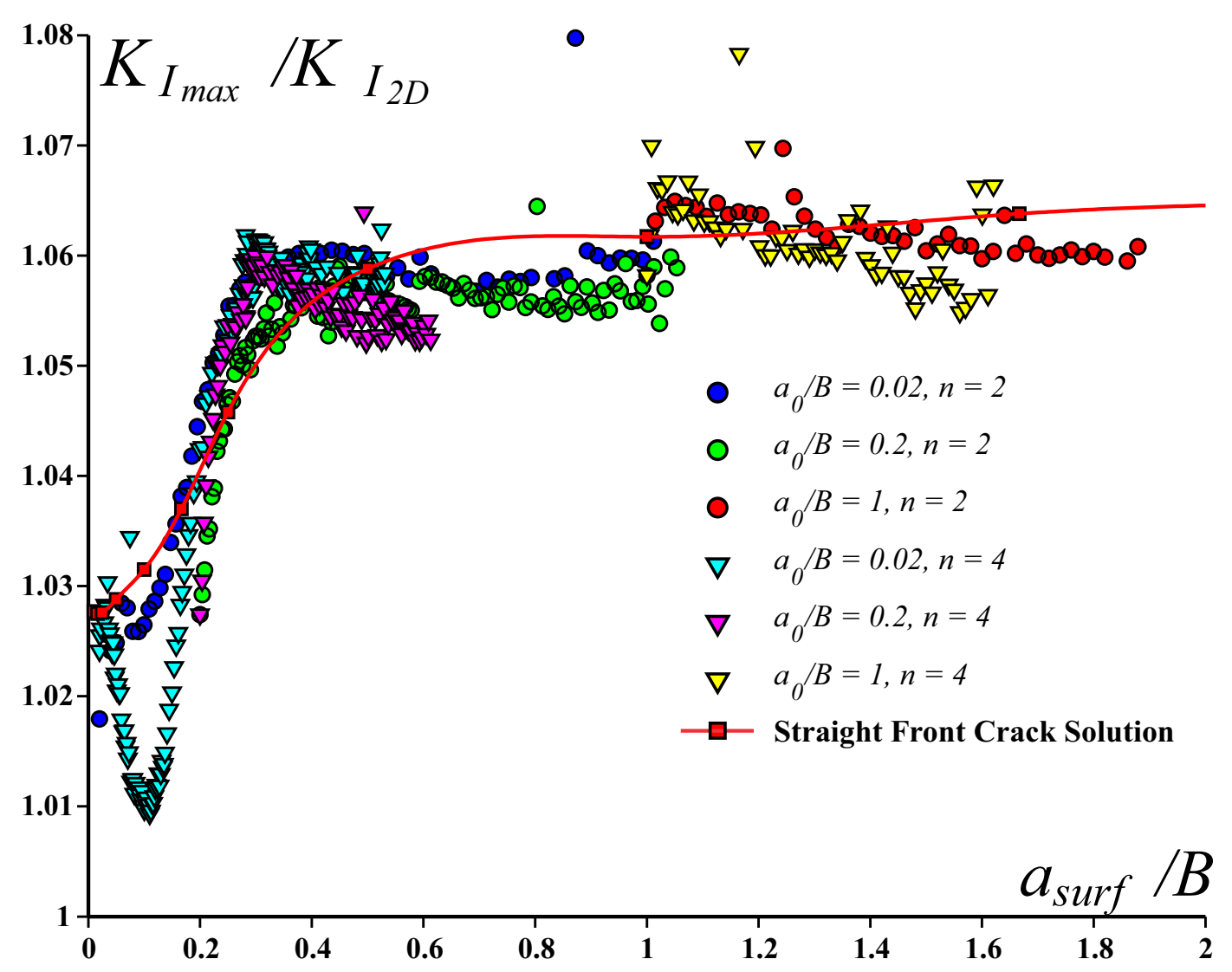

Figure $3.29-K_{I_{\max }} / K_{I_{2 D}}$ VS. $\left(a_{\text {surf }}-a_{0}\right) / B$

$27 \%$ lower than Bazant and Estenssoro predictions [2], in terms of difference from $90^{\circ}$. 


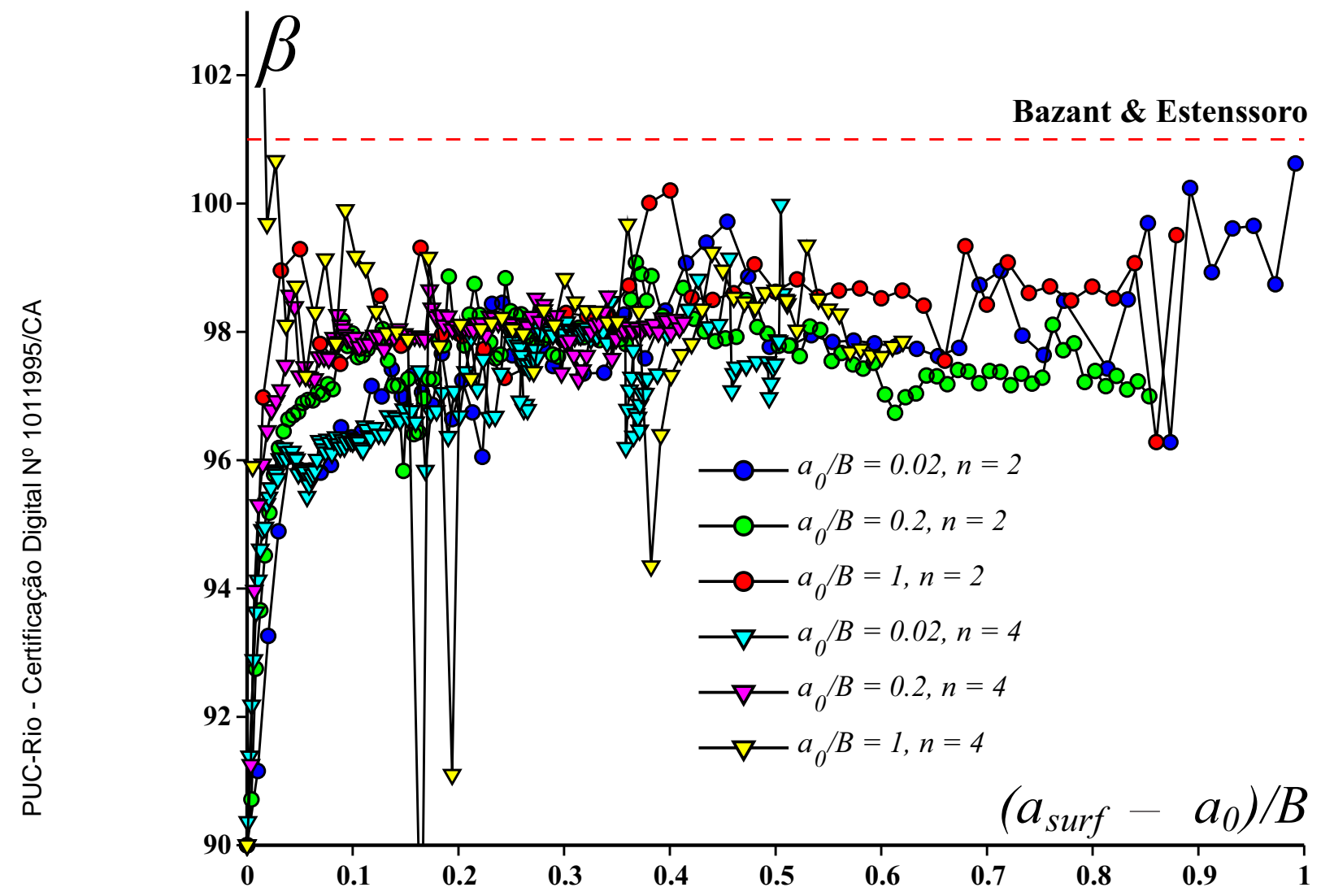

Figure 3.30 - Incidence angle $\beta$ at free surface with crack propagation 


\section{4 \\ Conclusions}

Several FE analyses were performed in order to observe 3D effects on the stress/strain fields close to notch and crack tips. The obtained results are aligned with the recent literature published on the matter. It was observed that the stress and strain concentration along the notch tip is variable, but the $\sigma_{y}$ gradient ahead of the notch tip can be obtained from the plane 2D solution. Also, the very same gradient causes an out-of-plane restriction on the material, which tends the notch tip to plane-strain condition as the tip radius grows sharper $(\rho \rightarrow 0)$. Concerning notched specimens design, the present results show that, for notches with reasonably low SCF's $\left(K_{t} \leq 3\right), 8 \%$ is a safe margin to estimate the maximum non-conservative error committed in the maximum stress and in Tresca's equivalent stress predictions, if 3D effects are ignored (i.e., if the $2 \mathrm{D}$ solution is used), and also that $\sigma_{\text {Mises }}$ at the notch tip is much less sensitive to $3 \mathrm{D}$ effects.

Further on, submodeling techniques were used to examine the 3D effects present in cracks on the edge of tensioned large plates with different thicknessto-crack-length $(B / a)$ ratios. Crack tip LE stress/strain fields were obtained taking into account the full load description, not restricted to $K$-field limitations and long crack assumptions. Also, the $T$-stress and the nominal stress influence were intrinsically computed. Both $K_{I}$ and $T$-stress were observed to vary along the crack front, presenting maximum $K_{I}$ values always higher than the $2 \mathrm{D}$ solution. The influence of the $B / a$ ratio on the $K_{I_{\mathrm{mp}}}$ was obtained, and it describes a smooth transient from the long crack solution presented in 22 (for $B / a \leq 0.1$ ) and the plane $2 \mathrm{D}$ solution (for $B / a \geq 100$ ). It was observed that $K_{I_{\max }}$ is always higher than the 2 D predictions.

The obtained stress gradients showed good agreement with $K$-solution for plates with $B / a$ between 0.1 and 100 up to $x=0.2 a$. From this point on, nonnegligible influence of nominal stress was observed. Considerable differences were observed in the restricted zone caused by short and long cracks. For long cracks, the reach of the restricted zone ahead of the crack tip is proportional to the plate thickness (limited to $x=0.4 B$ ), while for short cracks, the restricted zone is limited by the crack length (up to $x=10 a$ ). 
Finally, several crack growth simulations under Paris rule were performed. Initially straight cracks with different initial lengths $a_{0}$ and Paris exponent $n$ progressively curved their front during propagation, simultaneously flattening the $K_{I}$ distribution along the front. After some transient propagation, all cracks converged to the same regime crack propagation front, with tunneling depth close to $2.5 \%$ of $B$. 


\section{5}

\section{Bibliography}

1 Barsoum, R. S. Triangular quarter-point elements as elastic and perfectly-plastic crack tip elements. International Journal for Numerical Methods in Engineering 11, 1 (1977), 85-98.

2 Bazant, P. Z., And Estenssoro, L. F. Surface singularity and crack propagation. International Journal of Solids and Structures 15, 5 (1979), $405-426$.

3 Bowie, L. O. Analysis of edge notches in a semi-infinite region. Tech. Rep. AMRA TR 66-07, U.S. Army Materials Research Agency, Materials Engineering Division, september 1966.

4 Carter, B. J., Wawrzynek, P. A., and Ingraffea, A. R. Automated 3-d crack growth simulation. International Journal for Numerical Methods in Engineering 47 (2000), 229-253.

5 Creager, M., And Paris, P. C. Elastic field equations for blunt cracks with reference to stress corrosion cracking. International Journal of Fracture 3 (1967), 247-252. 10.1007/BF00182890.

6 Dassault Systemes. Abaqus v6.10 Manual.

7 de Castro, J. T. P., Meggiolaro, M. A., Miranda, A. C. O., Wu, H., And BenseddiQ, N. Prediction of fatigue crack initiation lives at elongated notch roots using short crack concepts. International Journal of Fatigue 42, 0 (2012), 172 - 182.

8 Glinka, G., And Newport, A. Universal features of elastic notch-tip stress fields. International Journal of Fatigue 9, 3 (1987), 143 - 150.

9 Henshell, R. D., And Shaw, K. G. Crack tip finite elements are unnecessary. International Journal for Numerical Methods in Engineering 9, 3 (1975), 495-507. 
10 Hwang, K., Leblond, B., Mouchrif, S. E., And Perrin, G. The tensile tunnel-crack with a slightly wavy front. International Journal of Solids and Structures 33, 14 (1996), 1995 - 2022.

11 Inglis, C. E. Stress in a plate due to the presence of cracks and sharp corners. Philosophical Transactions of the Royal Society series A 215 (1913), 119-233.

12 Ingraffea, A. R., And Manu, C. Stress-intensity factor computation in three dimensions with quarter-point elements. International Journal for Numerical Methods in Engineering 15, 10 (1980), 1427-1445.

13 Irwin, G. R. Analysis of Stresses and Strains Near the End of a Crack Traversing a Plate. J. Appl. Mech. 24 (1957), 361-364.

14 Kinsch, G. Die theorie der elastizitat und die bedurfnise der festigkeitslehre. Vereines Deutcher Ing. 42, 11 (1898), 797-807.

15 Lazarus, V., And LeBlond, J. Three-dimensional crack-face weight functions for the semi-infinite interface crack-i: Variation of the stress intensity factors due to some small perturbation of the crack front. Journal of the Mechanics and Physics of Solids 46, 3 (1998), 489 - 511.

16 Li, Z., Guo, W., And Kuang, Z. Three-dimensional elastic stress fields near notches in finite thickness plates. International Journal of Solids and Structures 37, 51 (2000), $7617-7632$.

17 Meggiolaro, M. A., Miranda, A. C. O., and de Castro, J. T. P. Short crack threshold estimates to predict notch sensitivity factors in fatigue. International Journal of Fatigue 29, 9-11 (2007), 2022 - 2031.

18 Nakamura, T., And Parks, D. Three-dimensional crack front fields in a thin ductile plate. Journal of the Mechanics and Physics of Solids 38, 6 (1990), $787-812$.

19 Nakamura, T., and Parks, D. M. Three-dimensional stress field near the crack front of a thin elastic plate. Journal of Applied Mechanics 55, 4 (1988), 805-813.

20 Pilkey, W., Pilkey, D., And Peterson, R. Peterson's stress concentration factors. John Wiley, 2008.

21 Pindra, N., Lazarus, V., And Leblond, J. B. Geometrical disorder of the fronts of a tunnel-crack propagating in shear in some heterogeneous 
medium. Journal of the Mechanics and Physics of Solids 58, 3 (2010), 281 -299 .

22 She, C., And Guo, W. The out-of-plane constraint of mixed-mode cracks in thin elastic plates. International Journal of Solids and Structures 44, 9 (2007), $3021-3034$.

23 She, C., And Guo, W. Three-dimensional stress concentrations at elliptic holes in elastic isotropic plates subjected to tensile stress. International Journal of Fatigue 29, 2 (2007), 330 - 335.

24 Sousa, R. A., Castro, J. T. P., Lopes, A. A. O., And Martha, L. F. On improved crack tip plastic zone estimates based on t-stress and on complete stress fields. Fatigue $\mathscr{G}$ Fracture of Engineering Materials $\&$ Structures 36 (2013), 25-38.

25 TAda, H., PARIs, P., AND IRWIn, G. The stress analysis of cracks handbook. No. v. 1 in The Stress Analysis of Cracks Handbook. Del Research Corp., 1973.

26 Wallin, K. The size effect in kic results. Engineering Fracture Mechanics 22, 1 (1985), $149-163$.

27 Williams, M. L. On the stress distribution at the base of a stationary crack. Journal of Applied Mechanics 24, 1 (1957), 109-114.

28 Wu, H., Imad, A., Benseddiq, N., de Castro, J. T. P., And Meggiolaro, M. A. On the prediction of the residual fatigue life of cracked structures repaired by the stop-hole method. International Journal of Fatigue 32, 4 (2010), 670 - 677.

$29 \mathrm{Wu}, \mathrm{Z}$. On the through-thickness crack with a curve front in center-cracked tension specimens. Engineering Fracture Mechanics 73, 17 (2006), 2600 2613.

30 Yang, Z., Kim, C. B., Cho, C., And Beom, H. G. The concentration of stress and strain in finite thickness elastic plate containing a circular hole. International Journal of Solids and Structures 45, 3-4 (2008), 713 731.

31 Youngdahl, C. K., And Sternberg, E. Three-dimensional stress concentration around a cylindrical hole in a semi-infinite elastic body. Journal of Applied Mechanics 33, 4 (1966), 855-865. 
32 Yu, P., Guo, W., She, C., and Zhao, J. The influence of poissons ratio on thickness-dependent stress concentration at elliptic holes in elastic plates. International Journal of Fatigue 30, 1 (2008), 165 - 171. 


\section{A}

\section{Notch FE models data}

This Appendix contains a summary of computational parameters from Notch Finite Element model runs.

Table A.1 - EH model runs: $\rho / a=1.0$

\begin{tabular}{|ccccccc|}
\hline Notch & $\rho / a$ & $B / \rho$ & elements & nodes & cpu time $(\mathrm{s})$ & clock time $(\mathrm{s})$ \\
\hline $\mathrm{EH}$ & 1 & 0.1 & 13932 & 61726 & 152.6 & 155 \\
$\mathrm{EH}$ & 1 & 0.2 & 13977 & 61915 & 153.6 & 155 \\
$\mathrm{EH}$ & 1 & 0.4 & 13797 & 61159 & 152.9 & 155 \\
$\mathrm{EH}$ & 1 & 0.6 & 14610 & 64682 & 156.3 & 158 \\
$\mathrm{EH}$ & 1 & 1 & 14910 & 65942 & 170 & 173 \\
$\mathrm{EH}$ & 1 & 1.5 & 15405 & 68021 & 155 & 195 \\
$\mathrm{EH}$ & 1 & 2 & 9073 & 40954 & 106.3 & 33 \\
$\mathrm{EH}$ & 1 & $10 \mathrm{~b}$ & 15630 & 68966 & 308.3 & 141 \\
$\mathrm{EH}$ & 1 & $10 \mathrm{c}$ & 15555 & 68651 & 267.7 & 117 \\
$\mathrm{EH}$ & 1 & $20 \mathrm{c}$ & 15255 & 6767391 & 275.7 & 137 \\
$\mathrm{EH}$ & 1 & $20 \mathrm{~d}$ & 29010 & 126102 & 1987.2 & 1277 \\
$\mathrm{EH}$ & 1 & $2 \mathrm{a}$ & 9169 & 41356 & 71.5 & 71 \\
$\mathrm{EH}$ & 1 & $2 \mathrm{~b}$ & 16497 & 72542 & 195.4 & 300 \\
\hline
\end{tabular}


Table A.2 - EH model runs: $\rho / a=0.25$

\begin{tabular}{|ccccccc|}
\hline Notch & $\rho / a$ & $B / \rho$ & elements & nodes & cpu time $(\mathrm{s})$ & clock time $(\mathrm{s})$ \\
\hline $\mathrm{EH}$ & 0.25 & 0.1 & 21538 & 94802 & 356.1 & 804 \\
$\mathrm{EH}$ & 0.25 & 0.2 & 44076 & 189967 & 1640 & 3396 \\
$\mathrm{EH}$ & 0.25 & 0.5 & 21654 & 94334 & 349.6 & 562 \\
$\mathrm{EH}$ & 0.25 & 0.7 & 21637 & 94260 & 382.8 & 588 \\
$\mathrm{EH}$ & 0.25 & 1 & 23862 & 103664 & 502.3 & 817 \\
$\mathrm{EH}$ & 0.25 & 1.5 & 23814 & 103466 & 518.1 & 926 \\
$\mathrm{EH}$ & 0.25 & 2 & 24006 & 104036 & 616.8 & 1076 \\
$\mathrm{EH}$ & 0.25 & 3 & 24438 & 105818 & 463.4 & 668 \\
$\mathrm{EH}$ & 0.25 & 4 & 26056 & 112574 & 503.5 & 797 \\
$\mathrm{EH}$ & 0.25 & 6 & 36418 & 156012 & 1000.7 & 1796 \\
$\mathrm{EH}$ & 0.25 & 6 & 36418 & 156012 & 924 & 1522 \\
$\mathrm{EH}$ & 0.25 & 8 & 23670 & 102650 & 414.8 & 611 \\
$\mathrm{EH}$ & 0.25 & $0.1 \mathrm{a}$ & 28270 & 123018 & 753.6 & 1236 \\
\hline
\end{tabular}

Table A.3 - EH model runs: $\rho / a=0.04$

\begin{tabular}{|ccccccc|}
\hline Notch & $\rho / a$ & $B / \rho$ & elements & nodes & cpu time $(\mathrm{s})$ & clock time $(\mathrm{s})$ \\
\hline EH & 0.04 & 0.04 & 20138 & 87166 & 5654.9 & 5720 \\
EH & 0.04 & 0.12 & 20503 & 88692 & 2375.4 & 2400 \\
EH & 0.04 & 0.24 & 20075 & 86935 & 988.4 & 1051 \\
EH & 0.04 & 0.4 & 21116 & 91219 & 846.4 & 858 \\
EH & 0.04 & 0.6 & 21018 & 90800 & 395 & 430 \\
EH & 0.04 & 0.8 & 21456 & 92579 & 349.5 & 361 \\
EH & 0.04 & 1.2 & 21533 & 92893 & 266.8 & 275 \\
EH & 0.04 & 2 & 40976 & 173479 & 437.7 & 525 \\
EH & 0.04 & 3 & 39364 & 166827 & 317.2 & 386 \\
EH & 0.04 & 4 & 20260 & 87672 & 151.7 & 160 \\
EH & 0.04 & 4 & 38664 & 163952 & 291.3 & 347 \\
\hline
\end{tabular}

Table A.4 - EH model runs: $\rho / a=0.01$

\begin{tabular}{|ccccccc|}
\hline Notch & $\rho / a$ & $B / \rho$ & elements & nodes & cpu time $(\mathrm{s})$ & clock time $(\mathrm{s})$ \\
\hline EH & 0.01 & 0.004 & 23301 & 102015 & 421.7 & 665 \\
EH & 0.01 & 0.006 & 22435 & 97520 & 489.9 & 681 \\
EH & 0.01 & 0.01 & 23241 & 100882 & 522.6 & 761 \\
EH & 0.01 & 0.02 & 22864 & 99275 & 467 & 670 \\
EH & 0.01 & 0.04 & 22714 & 98663 & 494.4 & 736 \\
EH & 0.01 & 0.06 & 22700 & 98622 & 1355.6 & 1447 \\
EH & 0.01 & 0.1 & 38341 & 163327 & 3363.5 & 3452 \\
EH & 0.01 & 0.2 & 38341 & 163327 & 3399.3 & 3520 \\
EH & 0.01 & 0.4 & 30423 & 130416 & 639.2 & 713 \\
EH & 0.01 & 0.6 & 29558 & 126878 & 405.5 & 474 \\
EH & 0.01 & 1 & 36241 & 154810 & 378.7 & 410 \\
EH & 0.01 & 3 & 86081 & 358609 & 909.9 & 1655 \\
\hline
\end{tabular}


Table A.5 - SE model runs: $\rho / a=1.0$

\begin{tabular}{|ccccccc|}
\hline Notch & $\rho / a$ & $B / \rho$ & elements & nodes & cpu time $(\mathrm{s})$ & clock time $(\mathrm{s})$ \\
\hline $\mathrm{SE}$ & 1 & 0.2 & 13300 & 59142 & 114.6 & 116 \\
$\mathrm{SE}$ & 1 & 0.3 & 13300 & 59142 & 110.8 & 112 \\
$\mathrm{SE}$ & 1 & 0.5 & 13300 & 59142 & 110.9 & 111 \\
$\mathrm{SE}$ & 1 & 0.7 & 13300 & 59142 & 109.2 & 110 \\
$\mathrm{SE}$ & 1 & 1 & 9715 & 43814 & 75.7 & 76 \\
$\mathrm{SE}$ & 1 & 2 & 8530 & 38602 & 58.5 & 59 \\
$\mathrm{SE}$ & 1 & 3 & 8625 & 39015 & 103.3 & 31 \\
$\mathrm{SE}$ & 1 & 5 & 8695 & 39295 & 62.6 & 63 \\
$\mathrm{SE}$ & 1 & 7 & 8620 & 38980 & 63.5 & 64 \\
$\mathrm{SE}$ & 1 & 10 & 8485 & 38413 & 59.6 & 60 \\
$\mathrm{SE}$ & 1 & 20 & 8365 & 37921 & 59.7 & 60 \\
$\mathrm{SE}$ & 1 & 30 & 8140 & 36976 & 53.3 & 53 \\
\hline
\end{tabular}

Table A.6 - SE model runs: $\rho / a=0.25$

\begin{tabular}{|ccccccc|}
\hline Notch & $\rho / a$ & $B / \rho$ & elements & nodes & cpu time $(\mathrm{s})$ & clock time $(\mathrm{s})$ \\
\hline $\mathrm{SE}$ & 0.25 & 0.02 & 21522 & 96424 & 355.2 & 498 \\
$\mathrm{SE}$ & 0.25 & 0.04 & 21666 & 97018 & 335.5 & 516 \\
$\mathrm{SE}$ & 0.25 & 0.06 & 21902 & 97986 & 347 & 481 \\
$\mathrm{SE}$ & 0.25 & 0.1 & 22622 & 100956 & 357.8 & 585 \\
$\mathrm{SE}$ & 0.25 & 0.2 & 27330 & 120602 & 442.5 & 685 \\
$\mathrm{SE}$ & 0.25 & 0.5 & 24984 & 109799 & 353.7 & 563 \\
$\mathrm{SE}$ & 0.25 & 1 & 27192 & 119129 & 494.9 & 714 \\
$\mathrm{SE}$ & 0.25 & 2 & 27336 & 119501 & 522.1 & 823 \\
$\mathrm{SE}$ & 0.25 & 3 & 27768 & 121283 & 527.4 & 790 \\
$\mathrm{SE}$ & 0.25 & 4 & 28440 & 124055 & 604 & 968 \\
$\mathrm{SE}$ & 0.25 & 6 & 25824 & 113264 & 469.6 & 724 \\
$\mathrm{SE}$ & 0.25 & 8 & 27000 & 118115 & 517.4 & 811 \\
\hline
\end{tabular}

Table A.7 - SE model runs: $\rho / a=0.04$

\begin{tabular}{|ccccccc|}
\hline \multirow{2}{*}{ Notch } & $\rho / a$ & $B / \rho$ & elements & nodes & cpu time $(\mathrm{s})$ & clock time $(\mathrm{s})$ \\
\hline $\mathrm{SE}$ & 0.04 & 0.02 & 19298 & 84693 & 381.4 & 559 \\
$\mathrm{SE}$ & 0.04 & 0.04 & 20016 & 87652 & 361.5 & 517 \\
$\mathrm{SE}$ & 0.04 & 0.12 & 19840 & 86320 & 328.9 & 532 \\
$\mathrm{SE}$ & 0.04 & 0.4 & 20994 & 91059 & 367.6 & 596 \\
$\mathrm{SE}$ & 0.04 & 0.8 & 21765 & 94248 & 385.8 & 643 \\
$\mathrm{SE}$ & 0.04 & 1.2 & 21748 & 94156 & 430.2 & 649 \\
\hline
\end{tabular}


Table A.8 - SE model runs: $\rho / a=0.01$

\begin{tabular}{|ccccccc|}
\hline Notch & $\rho / a$ & $B / \rho$ & elements & nodes & cpu time $(\mathrm{s})$ & clock time $(\mathrm{s})$ \\
\hline $\mathrm{SE}$ & 0.01 & 0.06 & 36168 & 154624 & 1200.1 & 2285 \\
$\mathrm{SE}$ & 0.01 & 0.1 & 36468 & 155833 & 2103.8 & 1028 \\
$\mathrm{SE}$ & 0.01 & 0.2 & 39488 & 168063 & 3482.4 & 3612 \\
$\mathrm{SE}$ & 0.01 & 0.4 & 39198 & 166897 & 1555.2 & 1605 \\
$\mathrm{SE}$ & 0.01 & 0.6 & 38323 & 163364 & 1158.5 & 1190 \\
$\mathrm{SE}$ & 0.01 & 1 & 36213 & 154804 & 576.8 & 589 \\
$\mathrm{SE}$ & 0.01 & 2 & 49808 & 210375 & 567.6 & 665 \\
$\mathrm{SE}$ & 0.01 & 4 & 43708 & 185045 & 318.9 & 714 \\
\hline
\end{tabular}




\section{B \\ Crack submodels runs}

Table B.1 - Crack submodels

\begin{tabular}{|ccccc|}
\hline B/a & elements & nodes & cpu time $(\mathrm{s})$ & wallclock time $(\mathrm{s})$ \\
\hline 0.1 & 23070 & 100027 & 700.1 & 1457 \\
0.2 & 23070 & 100027 & 642.3 & 1264 \\
0.4 & 17385 & 75398 & 386 & 861 \\
0.6 & 17385 & 75398 & 357.3 & 487 \\
1 & 23160 & 100358 & 634.1 & 1156 \\
2 & 23160 & 100358 & 319.4 & 515 \\
4 & 23970 & 103995 & 316.1 & 524 \\
6 & 24225 & 105207 & 1504.2 & 2168 \\
10 & 26955 & 117143 & 940.2 & 1334 \\
20 & 26955 & 117143 & 2487.8 & 610 \\
40 & 29655 & 129047 & 2882.8 & 756 \\
60 & 29610 & 128858 & 2740.7 & 745 \\
100 & 30495 & 132810 & 3119.2 & 908 \\
\hline
\end{tabular}

